\title{
The Role of the NIF in the Development of Inertial Fusion Energy
}

\author{
B. Grant Logan
}

March 16, 1995

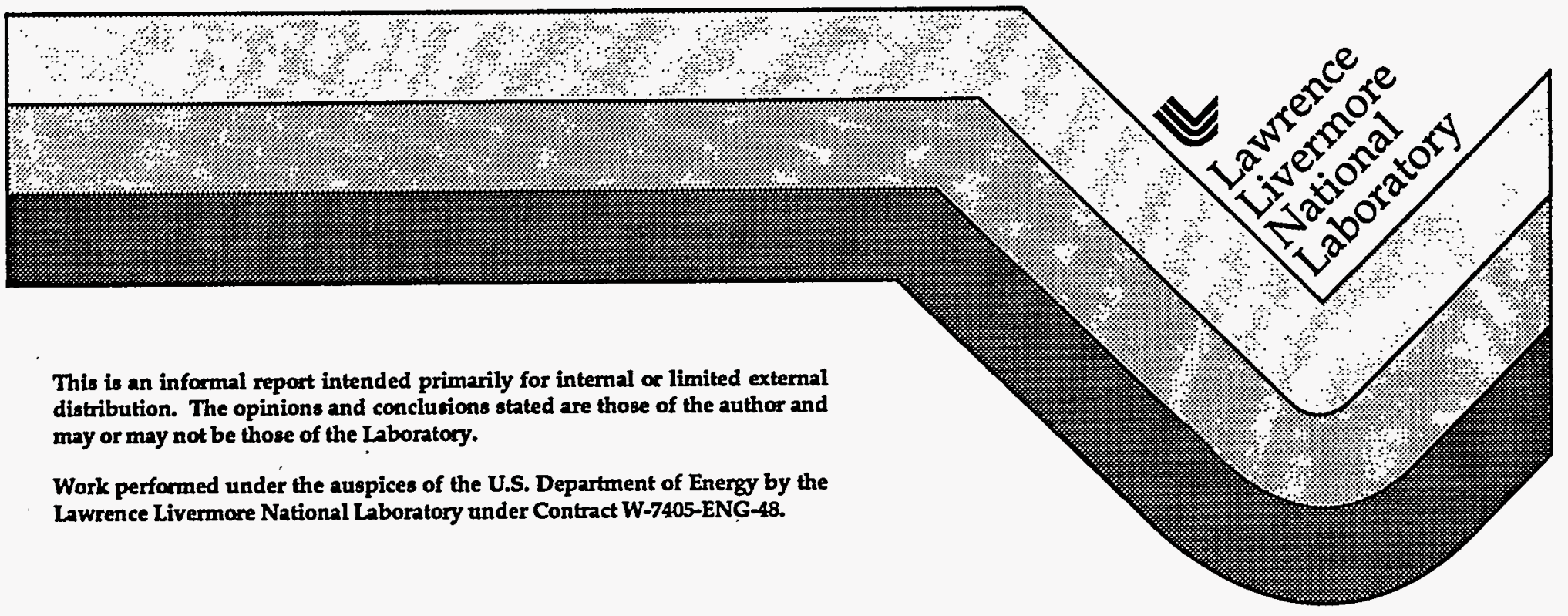




\section{DISCLAIMER}

This document was prepared as an account of work sponsored by an agency of the United States Government. Neither the United States Government nor the University of California nor any of their employees, makes any warranty, express or implied, or assumes any legal liability or responsibility for the accuracy, completeness, or usefulness of any information, apparatus, product, or process disclosed, or represents that its use would not infringe privately owned rights. Reference herein to any specific commercial product, process, or service by trade name, trademark, manufacturer, or otherwise, does not necessarily constitute or imply its endorsement, recommendation, or favoring by the United States Government or the University of California. The views and opinions of authors expressed herein do not necessarily state or reflect those of the United States Government or the University of California, and shall not be used for advertising or product endorsement purposes.

This report has been reproduced

directly from the best available copy.

Available to DOE and DOE conlractors from the Office of Scientific and Technical Information

P.O. Box 62, Oak Ridge, 'IN 37831

Prices available from (615) 576-8101, FTS 626-8401

Available to the public from the

National Technical Information Service

U.S. Department of Commerce

5285 Port Royal Rd.

Springfield, VA 22161 


\section{DISCLAIMER}

Portions of this document may be illegible in electronic image products. Images are produced from the best available original document. 


\title{
The Role of the NIF in the Development of Inertial Fusion Energy
}

\author{
B. Grant Logan
}

\subsection{Introduction}

Recent decisions (Key Decision 1) by DOE to proceed with the National Ignition Facility (NIF) and the first half of the Induction Systems Linac Experiments (ILSE) can provide the scientific basis for inertial fusion ignition and highrepetition heavy-ion driver physics, respectively. Both are critical to Inertial Fusion Energy (IFE). A conceptual design ${ }^{1-2}$ has been completed for a 1.8-MJ, 500-TW, 0.35- $\mu \mathrm{m}$-solid-state laser system, the NIF (Fig. 1-1). The NIF will demonstrate inertial fusion ignition and gain for national security applications, and for IFE development. It will support science applications using highpower lasers. ${ }^{3}$ The demonstration of inertial fusion ignition and gain, along with the parallel demonstration of the feasibility of an efficient, high-repetition-rate driver, would provide the basis for a follow-on Engineering Test Facility

\section{The National Ignition Facility-192 Beam}

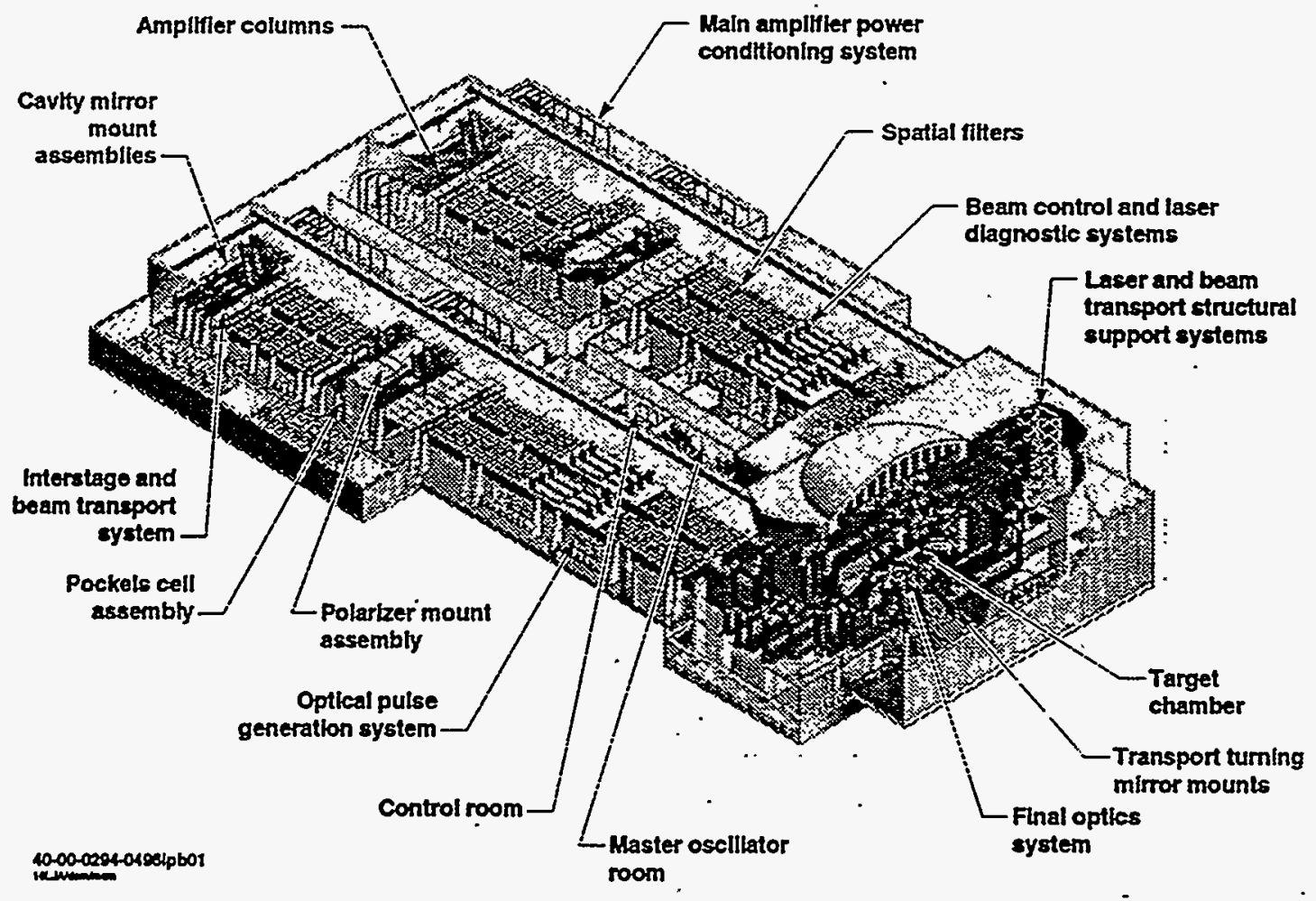

Figure 1-1. Schematic of the NIF. A solid-state laser delivers $1.8 \mathrm{MJ}$ of $0.35-\mu \mathrm{m}$ light to a target at the center of a 10-m-diam target chamber. 
(ETF) identified in the National Energy Policy Act of 1992 (Fig. 1-2). ${ }^{4}$ The ETF would provide an integrated testbed for the development and demonstration of the technologies needed for IFE power plants. In addition to target physics of ignition, the NIF will contribute important data on IFE target chamber issues, including neutron damage, activation, target debris clearing, operational experience in many areas prototypical to future IFE power plants, and an opportunity to provide tests of candidate lowcost IFE targets and injection systems. An overview of the NIF design and the target area environments relevant to conducting IFE experiments are described in Section 2. In providing this basic data for IFE, the NIF will provide confidence that an ETF can be successful in the integration of drivers, target chambers, and targets for IFE.

Magnetic fusion energy (MFE) and IFE are two primary and different approaches to fusion supported by the DOE, as called for by the
Fusion Policy Advisory Committee, ${ }^{5}$ and the National Energy Policy Act of $1992 .{ }^{4}$ Inertial fusion presents a very different set of physics and technology challenges from the Tokamak. Inertial fusion also has different characteristics that motivate its development, namely:

- Driver and Target Separability. The driver and target factory are separated from the fusion chamber environment, which prevents radiation and shock damage to the most complex plant equipment. The driver-chamber separability makes it possible for a single driver to drive multiple target chambers, providing flexibility in the required chamber pulse rate and lifetime, and allowing staged plant deployment of several fusion chambers to achieve low cost of electricity.

- Maturity of Inertial Fusion Physics. The Halite-Centurion underground tests have put to rest fundamental questions about

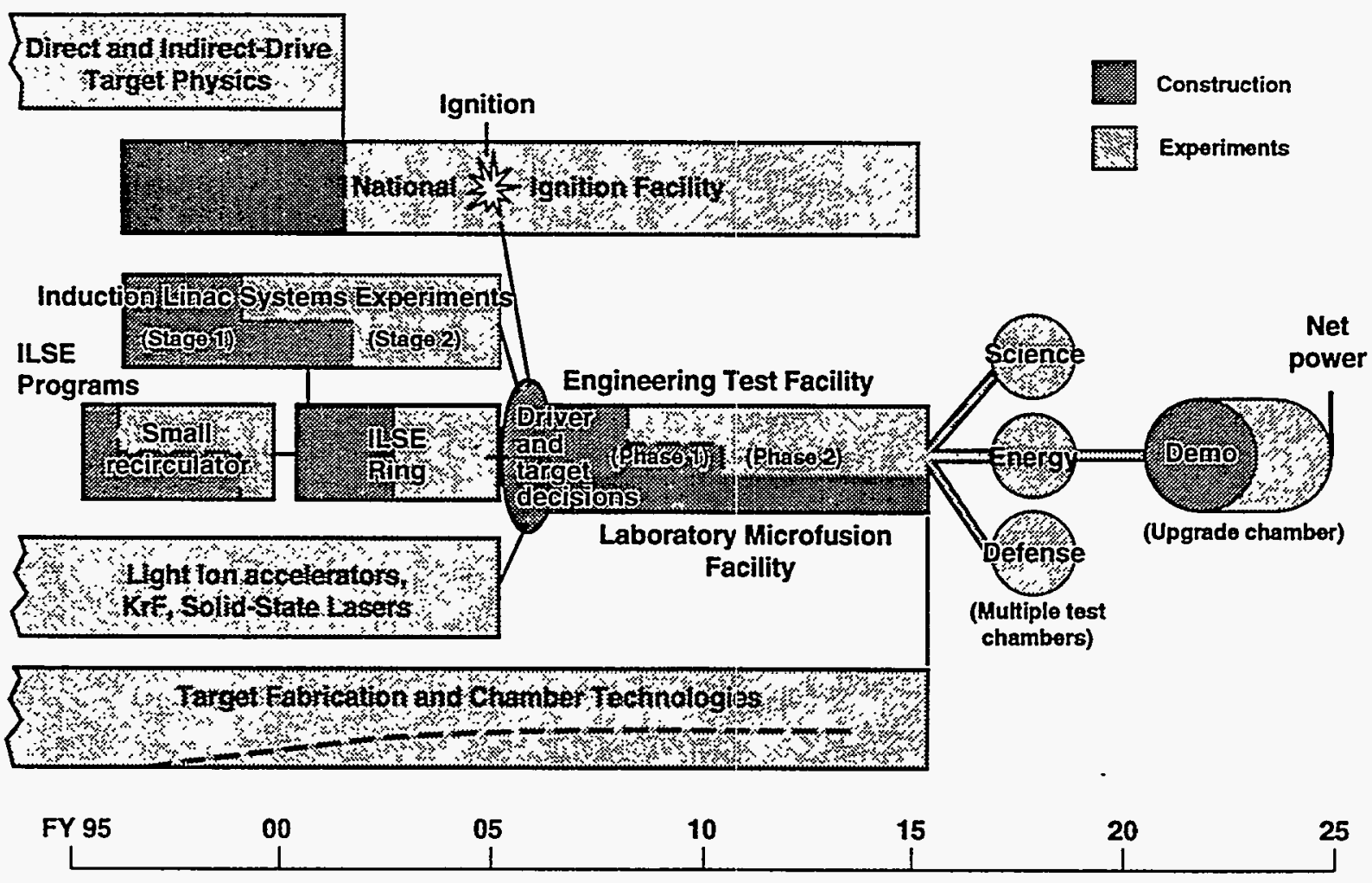

70-37-10943566C

Figure 1-2. IFE Development Plan including contributions by both Defense ICF Program and Energy Research. 
the scientific feasibility of inertial fusion ignition and gain. The progress in the Nova Technical Contract has shown thatinertial fusion computer codes can predict the most.important physics that would affect the gain of targets. When these computer models are fully validated by ignition tests in the NIF, they can be used to design improved targets for future IFE power plant designs.

- Flexibility in Fusion Chamber Design. IFE fusion chambers do not require a hard vacuum, allowing a greater range of materials and design choices to achieve very low activation and radioactive waste, and reduced maintenance. As an example, some IFE chamber designs protect the structural walls with thick, renewable fluid flows, which eliminates periodic replacement of internal structural components.

- Affordable Development. The development cost of IFE can be greatly minimized by sharing the use of NIF and other Defense Program assets for dual-use and benefit to defense and energy missions. As another example, a single, stageable driver could be used for a combined ETF/Laboratory Microfusion Facility (LMF) with multiple test chambers following the NIF, upgradable to a demonstration power plant (DEMO) after development of a suitable chamber technology at low fusion power.

This document explores the various ways the NIF can contribute to the realization of these potential IFE characteristics to provide an economical, environmentally benign energy source. By supporting NIF and IFE development, the United States can extend its present world leadership in inertial fusion expertise and industrial technology. IFE development supports the following US strategic energy goals:

- Promoting US world leadership in new energy technologies.

- Providing viable, clean energy sources that are alternatives to oil and other polluting fossil fuels.
- Reducing energy related emissions of greenhouse gases.

The primary mission of the NIF is defense, providing inertial fusion ignition and gain as a key R\&D capability for Science-Based Stockpile Stewardship (SBSS). In carrying out this primary mission, the NIF will also be contributing an essential capability for IFE. In addition, the NIF is to be a national user facility for several applications in the areas of weapons physics, weapons effects testing, inertial fusion energy, and basic science. There were four workshops held in 1994 to get input from representatives of each potential user group on how the NIF could be used to advance each area.

A workshop was convened at the University of California, Berkeley on February 22 to 24, 1994; it was nationally-attended by 61 participants from 17 US organizations and is described in Section 3. The workshop identified possible NIF contributions in four main IFE areas:

- Target Physics-Demonstration of inertial fusion ignition and burn, comparison of direct and indirect drive performance, optimization of capsule physics and hohlraum geometry (soft- $x$ ray transport) for indirect-drive ion targets.

- Target Chamber DynamicsUnderstanding the materials responses to target emissions of neutrons, soft $x$-rays, and debris. Data to benchmark hydrodynamic and radiationhydrodynamic codes used for chamber clearing.

- IFE Fusion Power Technologies-Integrated operation of prototypical IFE fusion systems, for low fluence, single shots. Calibration of neutron transport, heating, activation. Target tritium burnup, transport and recovery. Demonstration of safe operation and management of radioactive inventories.

- Target Systems-Testing performance of candidate mass-produced IFE targets, target injection methods, and tracking precision.

Most of these IFE-specific experiments can be performed on the NIF after first ignition is demonstrated. The experiments can also be 
performed while the ETF/LMF driver is being built to provide guidance to the target and fusion chamber designs of the ETF. We explored generic experiments that would serve to benchmark codes and improve our understanding of basic phenomena. More detailed designs of these experiments will be required to further define IFE-specific development needs and to insure compatibility with the NIF target chamber design (Fig. 1-3), prior to fielding them. A preliminary evaluation, however, indicates that the proposed experiments are generally compatible with the flexibility of the NIF conceptual design. ${ }^{3}$

Section 3 reviews the results of the NIF-IFE workshop in these four topical areas.

There is a particularly close correspondence of the NIF indirect-drive target design and the target physics it will demonstrate in the ICF ignition campaign, with the features and requirements for indirect-drive, heavy ion targets designed for IFE power plants. ${ }^{6}$ Section 4 explores this connection in more detail. It also describes how the NIF experiments can be used to do the following: 1) further optimize specific heavy-ion target features (and thus help establish ETF ion-driver requirements); 2) determine the required precision needed for low-cost manufacture of targets for future IFE power plants.

The role of the NIF within an overall IFE development plan to substantially resolve all target physics issues and provide the design basis for ETF is described in Section 5. Section 5 also includes discussions on the following: 1) the contributions that the NIF Core Science and Technology programs will make to IFE and 2) the role of NIF in IFE laser driver development.

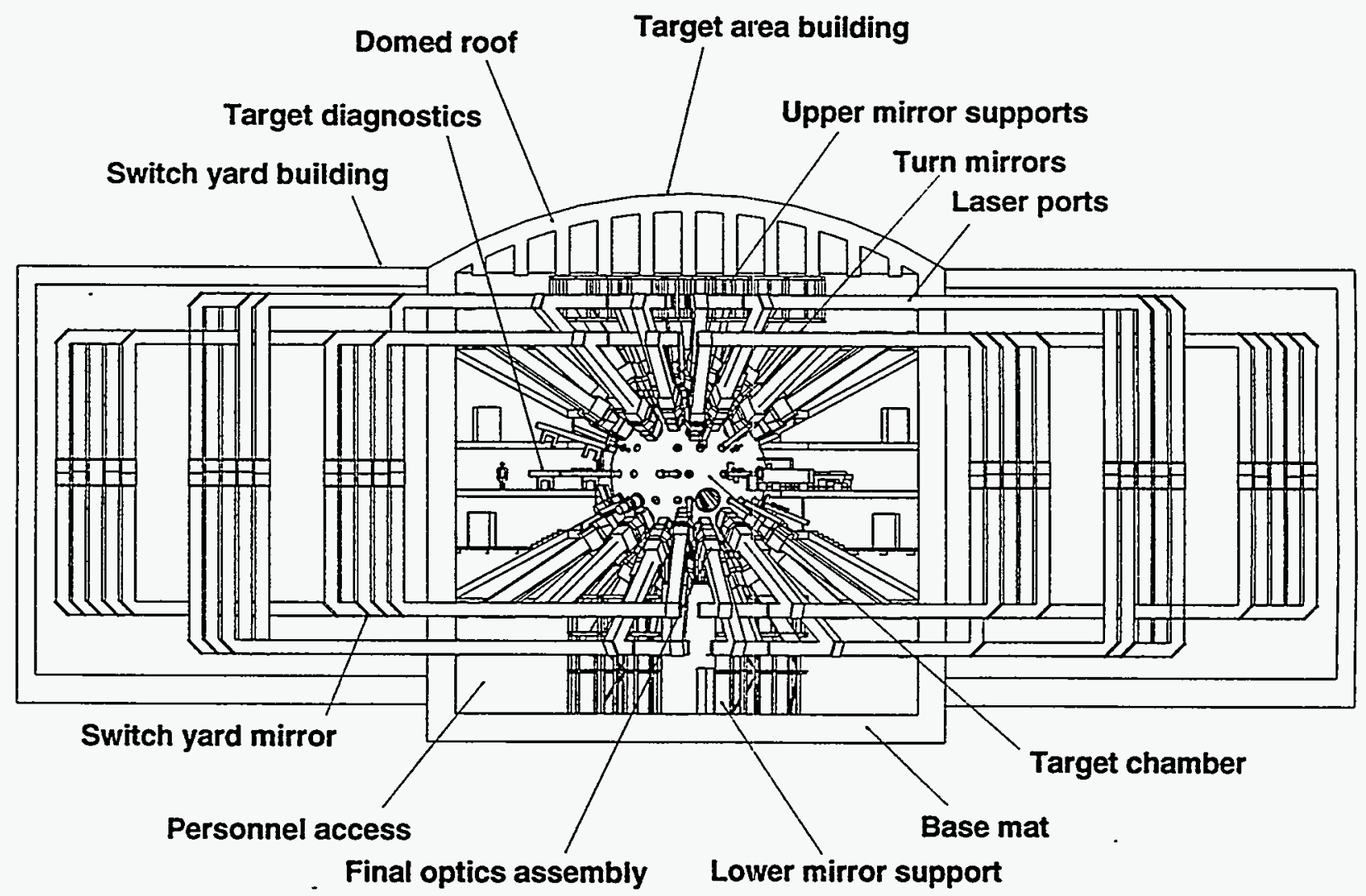

40-00-0394-1191

Figure 1-3. NIF target area illustrating beam geometry and design features. 
A discussion of the relationship between IFE. and the proliferation of nuclear weapons capabilities comprises Section 6 . This builds on the recent assessments of NIF's role in proliferation. Conclusionis on our assessments of the role of NIF for IFE development are summarized in Section 7.

\section{References}

1. National Ignition Facility Conceptual Design Report, UCRL-Prop-117093, NIFLLNL-94-113, L-16973-1 (May 1994).
2 J. A. Paisner, E. M. Campbell, and W. J. Hogan, "The National Ignition Facility Project," Fusion Technol., 26, 755 (1994).

3. M. Tobin, G. Logan, A. Anderson, and T. Diaz De La Rubia, "Use of the National Ignition Facility for the Development of Inertial Fusion Energy," Fusion Technol., 26, 857 (1994).

4. "The National Energy Policy Act of 1992," Public Law 102-486.

5. D. D.-M. Ho, J. A. Harte, M. Tabak, "Radiation-Driven Targets for Heavy-Ion Fusion," Proc. of 15th IAEA Int. Conf. on Plasma Phys. and Controlled Fusion Research (Seville, Spain, Sept. 6 to Oct. 1, 1994) IAEA-CN-60/B-P-13 (1994). 


\subsection{Overview of NIF Target Area and Environments}

\subsection{Introduction}

The NIF target area is designed for ICF experiments to achieve the scientific goal of fusion ignition. The target area will provide appropriate conditions before, during, and after each shot. The repeated introduction of large amounts of laser energy into the chamber and emission of fusion energy from a cryogenic target, represent new challenges in ICF facility design. Prior to a shot, the facility provides proper illumination geometry, target chamber vacuum, and a stable platform for the target and its diagnostics. During a shot, the impact of the energy introduced into the chamber is minimized, and workers and the public are protected from excessive prompt radiation doses. After the shot, the residual radioactivation is managed to allow required accessibility. Tritium and other radioactive wastes are controlled and disposed. Diagnostic data are also retrieved, and the facility is readied for the next shot.

While the largest anticipated yield is $\sim 20 \mathrm{MJ}$, the target area is designed to accommodate a maximum credible yield of $45 \mathrm{MJ}$. The assumed annual number of target experiments for the ignition campaign is shown in Table 2-1 as a function of the shot energy output (i.e., the sum only $10-20 \%$ of the experiments will produce fusion yields (in square brackets) ranging from of the laser energy plus fusion yield). Note that 0.1 to $20 \mathrm{MJ}$. After ignition is achieved, the anticipated increased use of NIF for its applications (Stockpile Stewardship, Basic Sciences, IFE, and Radiation Effects) may call for a new operating envelope of shots and associated yields.

The NIF is configured in a "U-shape" with the Target Area at the base of the "U." This arrangement preserves the flexibility of adding
Table 2-1. Assumed annual NIF target experiments.

\begin{tabular}{|c|c|c|}
\hline \multicolumn{2}{|c|}{$\begin{array}{l}\text { Shot energy, M] } \\
\text { [fusion yield] }\end{array}$} & Number of shots \\
\hline 1.0 & & $250-500$ \\
\hline 1.5 & & $250-500$ \\
\hline 1.8 & & $50-150$ \\
\hline 1.9 & {$[0.1]$} & 100 \\
\hline 6.8 & {$[5.0]$} & 35 \\
\hline 21.8 & {$[20.0]$} & 10 \\
\hline
\end{tabular}

more target areas in the future. The NIF target chamber is a 10-cm-thick aluminum sphere with a 5-m radius (Fig. 1-3). The chamber and target area support structures will become radioactive following shots producing neutrons. Shielding on the chamber exterior is provided to reduce activation of structures and to reduce the dose from the radioactive decay of the chamber. Access to the chamber area is controlled and monitored for up to several days after shots producing fusion yields of more than a few MJ. The maximum annual occupational dose is less than $0.5 \mathrm{rem}$, which is $1 / 10$ of allowable occupational dose given in DOE Order 6400.11. To provide the required stability and radiation levels at a reasonable cost, the target chamber support structure is made from aluminumreinforced, borated concrete.

The target positioner will be the chamber component closest to the target, and it will see the largest radiation fluences. For the first year or two, the NIF experimental plan calls for targetlike disks and nonigniting, noncryogenic hohlraums held in place with the NIF target 
positioner using a target stalk, as on Nova. After this initial series of experiments, the target positioner will provide cooling for cryogenic targets used for fusion yield shots. The front surface of the target positioner will be protected with a layer of frost (probably water), which will be ablated by target emissions and be pumped out as a noncondensible gas after the shot. The cryogenic target support system (Fig. 2-1) uses two capillary tube loops extending $-10-20 \mathrm{~cm}$ from the end of the positioner, filled with $\mathrm{He}$

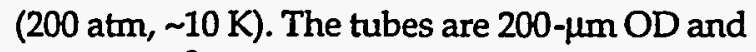
125- $\mu \mathrm{m} \mathrm{ID.2}$

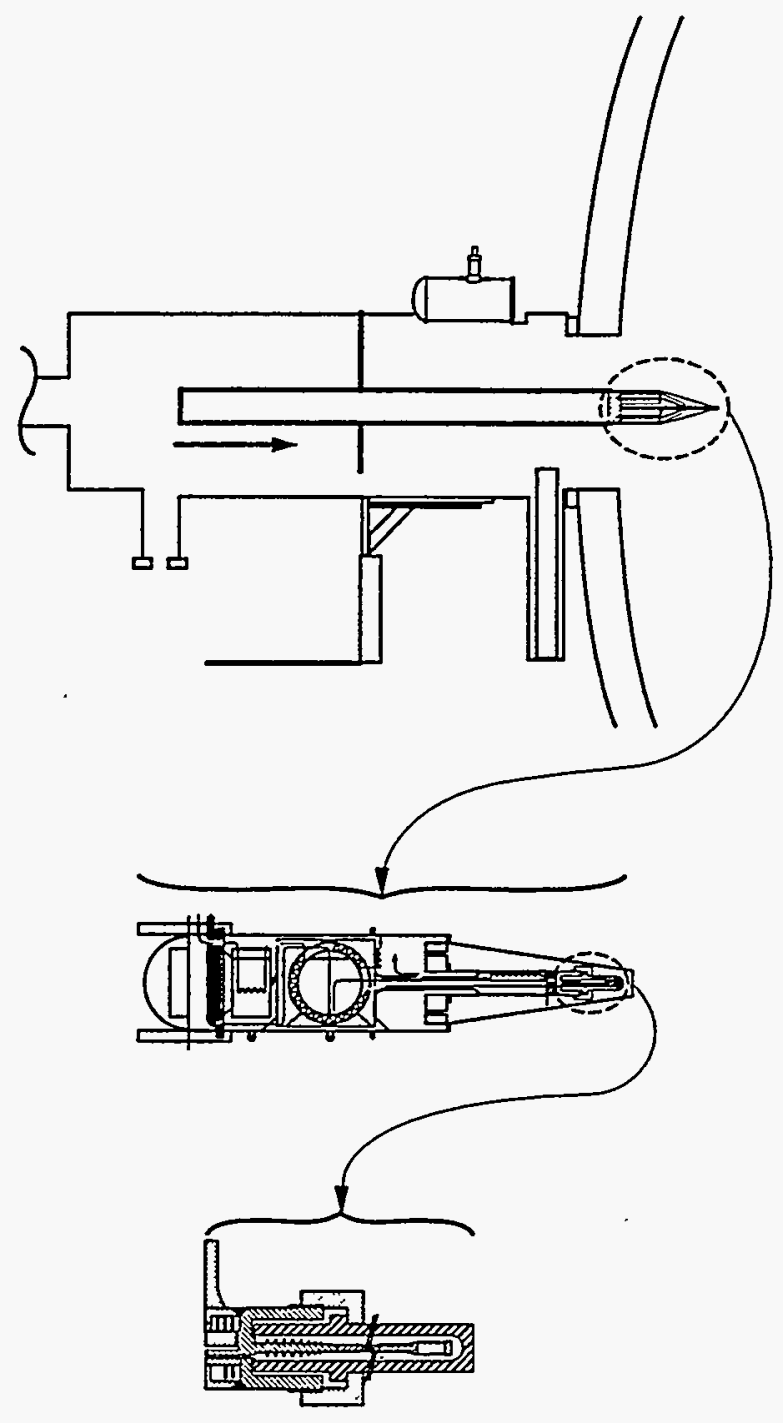

70.37-1294.39240001

Figure 2-1. Cryogenic target support system.
Twelve-inch diameter manipulator tubes are mounted on the chamber near its equator to allow insertion of diagnostics and experimental apparatus. Diagnostics not designated a specific beam port will be adapted for insertion into these tubes. Experiments not supporting ignition, such as samples to be exposed to neutrons, $x$ rays, and/or debris for chamber dynamics studies, can also be introduced through this method. Larger experimental packages will be accommodated by the large ports at the poles of the chamber, and on the equator.

The annual tritium throughput will be $300-600 \mathrm{Ci}$, with $2 \mathrm{Ci}$ of tritium in each cryogenic target intended for DT yield. After the admission of a DT target into the chamber for an experiment producing yield, tritium is nearly entirely removed from the chamber through the vacuum system where it is trapped on a molecular sieve. At periodic intervals, filters are removed and disposed of in accordance with DOE regulations. The largest tritium inventory in the facility at any time will not exceed $300 \mathrm{Ci}$. The annual routine tritium release will be $\leq 10 \mathrm{Ci}$.

Decontamination of surface tritium and activated debris will be accomplished employing carefully adapted $\mathrm{CO}_{2}$ cleaning technology. Robotic systems will perform in-chamber cleaning functions, while a near-chamber decontamination facility (glovebox) will support cleaning components, tools, and equipment. Special vacuum systems associated with the $\mathrm{CO}_{2}$ cleaners will recover the target and other debris $(\sim 100 \mathrm{~g} / \mathrm{yr})$ for safe storage and periodic removal.

\subsection{Target Emissions}

To plan IFE-relevant experiments for NIF, it is necessary to know the radiation environment produced by ignition and nonignition NIF targets. In this section, we describe the radiation environment at several locations as a function of the target shot energy, which is equal to the sum of the laser energy and fusion yield. The radiation includes high-energy fusion neutrons, $x$ rays, debris, and shrapnel. 
Neutrons-Neutrons will be produced in two ways: 1) from exploding pusher experiments that are frequently used to calibrate neutron diagnostics, and 2) from indirect-drive ignition targets. Nova today can-produce $2 \times 10^{13}$ neutrons (14 MeV) with an exploding pusher target. With 1.8 MJ, NIF could produce $\sim 10^{15}$ neutrons and perhaps more with improved capsules. When a target ignites and gives gain, $3.55 \times 10^{17}$ neutrons are released per MJ of fusion yield. Table 2-2 shows the fluences calculated for the target positioner $(20 \mathrm{~cm})$, chamber first wall $(5 \mathrm{~m})$, and debris shield $(6.75 \mathrm{~m})$. The neutron fluence is large enough that portions of the cryogenic target support tubes are melted, and post-shot radioactivity levels are high enough to require shielding (40-cm-borated concrete). The neutron fluence at the fused silica debris shields is insignificant with respect to radiation damage for the time period of the ignition campaign. ${ }^{2}$ An expanded program to support applications could produce 5-10x more neutrons per year. This is possibly an issue for optics and is being assessed.

$X$ rays $-X$-ray environments for testing material response can be created two ways: 1 ) by using the laser energy incident on a disk or into an empty hohlraum, and 2) by igniting and burning an ICF target. Table 2-3 describes the $x$-ray emissions relevant for planning IFE experiments. These values assume that $50 \%$ of the laser energy is converted into $x$ rays and the remainder into debris energy. Likewise, of the $20 \%$ of the fusion yield that is not neutrons, half is assumed to contribute to $x$ rays and half to debris. This latter assumption is based on averaging results from many calculations but requires further experimental and theoretical confirmation. The assumed spectrum is an average between disk and Nova hohlraum ' spectra. The Lambertian distribution of radiation from either a laser entrance hole or the surface of a heavy-metal disk results in a range of fluences on the first wall for any given target. Since some deviation from a truly Lambertian (cosine) distribution will occur, the fluences given in Table 2-3 are conservative. The range of fluences for the debris shields column spans this distribution at the two cone angles on which the beams are located. The $x$-ray pulse length, expected to be a few to several nanoseconds, is taken to be $1 \mathrm{~ns}$. This is a conservative assumption because $1 \mathrm{~ns}$ is too short a period for thermal conduction of $x$-ray energy away from the deposition area.

Debris-Table 2-4 describes the assumed fluences and energies of target debris. These values assume that $50 \%$ of the laser energy is converted into $x$ rays and the remainder is in debris energy. Likewise, of the $20 \%$ of the fusion yield that is not neutrons, half is assumed to contribute to $x$ rays and half to debris. The average ion energy is smaller for fusion yield shots (shot energy $>1.8 \mathrm{MJ}$ ) than for nonyield shots due to the additional cryogenic materials over which to distribute the shot energy. A debris pulse length of $30 \mu \mathrm{s}$ is assumed for all cases up

Table 2-2. Assumed neutron fluences at the target positioner, first wall, and debris shields.

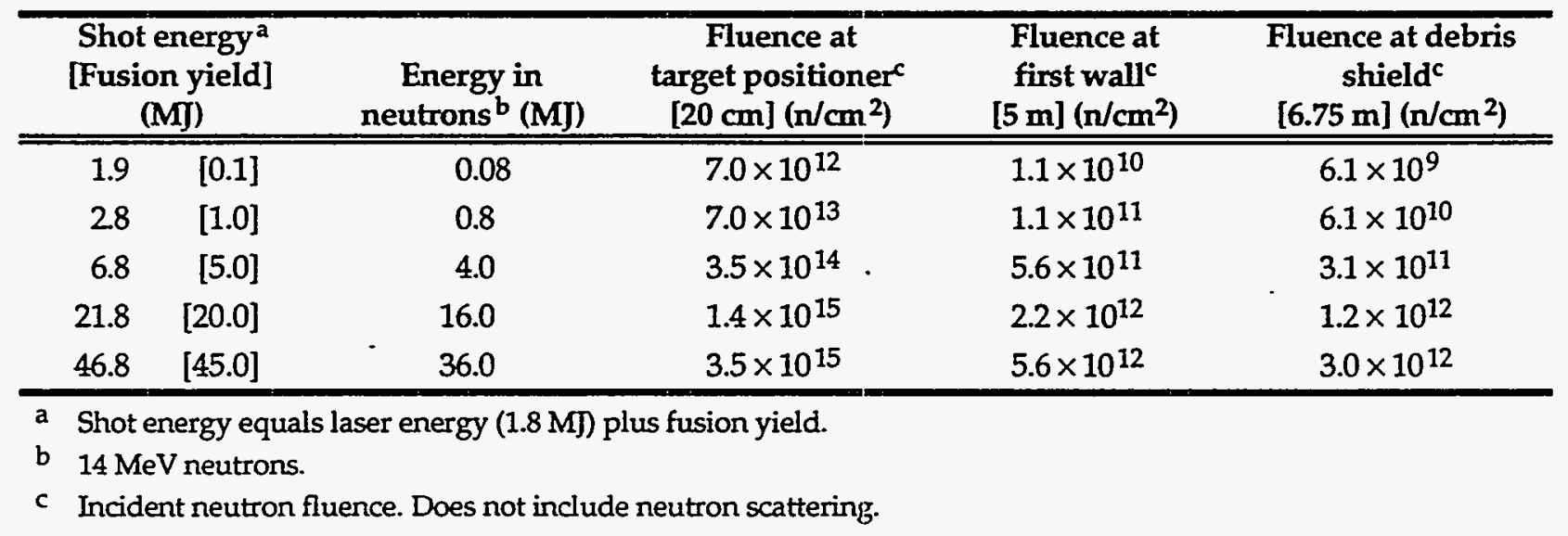


Table 2-3. Assumed NIF $x$-ray fluences and other characteristics.

\begin{tabular}{|c|c|c|c|c|c|c|}
\hline $\begin{array}{c}\text { Shot energy }^{\mathrm{a}} \\
\text { [Fusion yield] } \\
\text { (MJ) }\end{array}$ & $\begin{array}{c}\text { Energy in } \\
\text { - débris } \\
\text { (MJ) } \\
\end{array}$ & $\begin{array}{c}\text { Average ion } \\
\text { energy } \\
\text { (keV) }\end{array}$ & $\begin{array}{c}\text { Pulse } \\
\text { duration } \\
\text { (us) }\end{array}$ & $\begin{array}{c}\text { Fluence at } \\
\text { target } \\
\text { positioner } \\
{[20 \mathrm{~cm}]^{b}} \\
\left(\mathrm{~J} / \mathrm{cm}^{2}\right) \\
\end{array}$ & $\begin{array}{c}\text { Fluence at } \\
\text { first wallc } \\
{[5 \mathrm{~m}]\left(\mathrm{J} / \mathrm{cm}^{2}\right)}\end{array}$ & $\begin{array}{c}\text { Fluence at } \\
\text { debris } \\
\text { shield } \\
{[6.75 \mathrm{~m}]} \\
\left(0 / \mathrm{cm}^{2}\right) \\
\end{array}$ \\
\hline 1.0 & 0.50 & 12 & 30 & 99.5 & $0.13-0.65$ & 0.07 \\
\hline 1.5 & 0.75 & 18 & 30 & 149 & $0.19-0.95$ & 0.11 \\
\hline 1.8 & 0.90 & 22 & 30 & 179 & $0.23-1.15$ & 0.13 \\
\hline [0.1] & 0.91 & 2.4 & 30 & 179 & $0.24-1.20$ & 0.14 \\
\hline [1.0] & 1.0 & 2.7 & 30 & 199 & $0.26-1.30$ & 0.15 \\
\hline [5.0] & 1.4 & 3.8 & 30 & 279 & $0.59-3.00$ & 0.33 \\
\hline [20.0] & 2.9 & 7.9 & 30 & 577 & $1.00-5.00$ & 0.53 \\
\hline [45.0] & 5.4 & 14.7 & 30 & 1075 & $1.60-9.00$ & 0.88 \\
\hline
\end{tabular}

a Shot energy equals laser energy plus fusion yield.

b Target positioner at $20 \mathrm{~cm}$ from target with isotropic fluence.

c Indicated range at first wall is from isotropic average fluence to 5 times greater for jetting.

d Debris shield fluence is for an isotropic distribution.

Table 2-4. Assumed NIF x-ray fluences and other characteristics.

\begin{tabular}{|c|c|c|c|c|c|c|}
\hline $\begin{array}{l}\text { Shot energy }{ }^{\mathrm{a}} \\
\text { [Fusion yield] } \\
\text { (M]) }\end{array}$ & $\begin{array}{c}\text { Energy in } \\
\text { x rays } \\
\text { (MJ) }\end{array}$ & $\begin{array}{c}\begin{array}{c}\text { Color } \\
\text { temperature } \\
(\mathrm{eV})\end{array} \\
\end{array}$ & $\begin{array}{c}\text { Pulse } \\
\text { duration } \\
\text { (ns) } \\
\end{array}$ & $\begin{array}{c}\text { Fluence at } \\
\text { target } \\
\text { positioner } \\
{[20 \mathrm{~cm}]\left(0 / \mathrm{cm}^{2}\right)}\end{array}$ & $\begin{array}{l}\text { Fluence at } \\
\text { first wall } \\
{[5 \mathrm{~m}]\left(0 / \mathrm{cm}^{2}\right)}\end{array}$ & $\begin{array}{c}\text { Fluence at } \\
\text { debris } \\
\text { shield }^{\mathrm{d}} \\
{[6.75 \mathrm{~m}]} \\
\left(\mathrm{J} / \mathrm{cm}^{2}\right) \\
\end{array}$ \\
\hline 1.0 & 0.50 & 170 & 1 & 99.5 & $0.16-0.32$ & $0.10-0.16$ \\
\hline 1.5 & 0.75 & 170 & 1 & 149 & $0.24-0.48$ & $0.15-0.24$ \\
\hline 1.8 & 0.90 & 170 & 1 & 179 & $0.29-0.58$ & $0.19-0.29$ \\
\hline$[0.1]$ & 0.91 & 170 & 1 & 179 & $0.29-0.58$ & $0.19-0.29$ \\
\hline [1.0] & 1.0 & 200 & 1 & 199 & $0.32-0.64$ & $0.21-0.32$ \\
\hline [5.0] & 1.4 & 250 & 1 & 279 & $0.45-0.90$ & $0.29-0.45$ \\
\hline [20.0] & 2.9 & 350 & 1 & 577 & $0.92-1.80$ & $0.58-0.92$ \\
\hline [45.0] & 5.4 & 350 & 1 & 1075 & $1.70-3.50$ & $1.10-1.70$ \\
\hline
\end{tabular}

a Shot energy equals laser energy plus fusion yield.

b Target positioner at $20 \mathrm{~cm}$ from target with isotropic $x$-ray fluence.

c Indicated range at first wall is from isotropic average fluence to Lambertian peak fluence.

d Indicated range at debris shield is for Lambertian distribution at $52^{\circ}$ and $27^{\circ}$ rings. 
to $20 \mathrm{MJ}$ based on calculations using TSUNAMI, a UC Berkeley gas dynamics code. ${ }^{3}$ Although some preliminary calculations suggest timedependent effects (debris interaction with $x$-ray blow-off from the target positioner and wall) reduces the debris impact, we neglect it here to be conservative. 4 The range in first wall fluences addresses the "jetting" phenomena observed with Nova hohlraums.

Shrapnel-Shrapnel (defined as material in a liquid or solid state) will be produced from structures in the vicinity of the target which are not vaporized by the laser or yield energy. The primary sources will be the target support stalk for non-cryogenic targets and the stainless steel cooling tubes for the cryogenic type. These structures are heated and broken up by neutrons, $x$ rays, and target debris. Both solid fragments and liquid droplets will be propelled radially at velocities of 300 to $3000 \mathrm{~m} / \mathrm{s}$. The anticipated size distribution for fragmentation of the stainless steel tubes is shown in Fig. 2-2.5

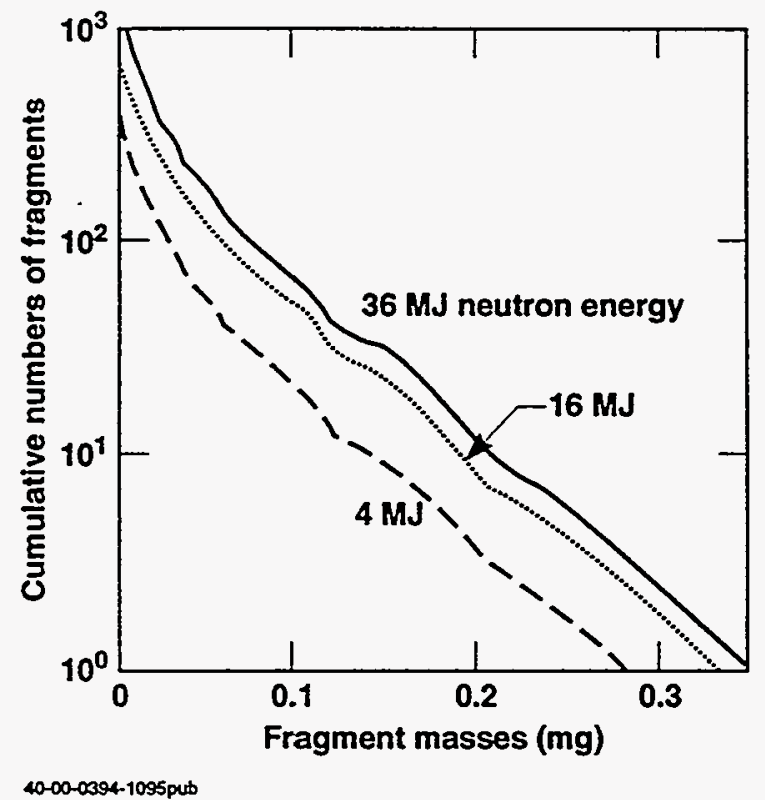

Figure 2-2. Anticipated size distribution for fragmentation of cryogenic stainless steel tubes for various fusion yields.

\section{References}

1. R. Warren, "Nova-Nova Upgrade Cryogenic Targets Thermal Design Approach," ' Engineering Note ENE 90-068 (October 1990).

2 M.S. Singh, "Effects of $14-\mathrm{MeV}$ Neutrons on a Fused-Silica Lens," Internal LLNL Memo (July 27, 1988).

3. P. F. Peterson, "NIF 1.8 MJ No Yield Response," Presentation at NIF First Wall Workshop \#4 (Feb. 10, 1994).

4. R. R. Peterson, "University of Wisconsin Capabilities to Model NIF Target Chamber Phenomena and Initial NIF Vaporization Results," Presentation at NIF First Wall Workshop \#2, LLNL (Dec. 14, 1993).

5. R. E. Tokheim, L. Seaman, and D. R. Curran, "Threats from Radiation Effects in the Proposed Nova Upgrade," SRI Final Report (Sept. 1992). 


\subsection{Review of the 1994 Workshop on the Use of NIF for IFE}

\subsection{Overview of the NIF-IFE Workshop}

\subsubsection{Introduction}

Workshop attendees represented diverse groups of experts and contributors (national stakeholders in IFE development), ICF and MFE fusion labs, universities, and private US corporations and small businesses.

Approximately 60 participants were asked to consider how the NIF can and should be used to advance Inertial Fusion Energy development. These potential users of the NIF showed great enthusiasm for the experimental opportunities for IFE development, exhibiting sparkling originality, innovation, individual initiative, and cross-disciplinary teamwork. The workshop was cosponsored and organized by LLNL and UC Berkeley; it was structured to include plenary and parallel working sessions. The first plenary session was devoted to introductory talks by the workshop organizers; it provided an overview of the NIF design, capabilities, and baseline diagnostics. We then asked the workshop participants to identify key credibility and development issues for IFE in four areas. Each area was examined in detail in parallel sessions [session chairmen are named in brackets]:

Target Physics [Roger Bangerter, LBL]Issues related to the design and performance of IFE targets;

Chamber Dynamics [Robert Tokheim, SRI]Issues in IFE chambers resulting from the deposition of $x$ rays and debris;

Inertial Fusion Power Technology [Mohammed Abdou, UCLA]-Issues for energy conversion, tritium breeding and processing, and radiation shielding, interactions of neutrons with materials, and chamber design;

Target Systems [Ken Schultz, General Atomics]-Issues related to automated, highproduction-rate manufacture of low-cost targets for IFE, target handling and transport, target injection, tracking, and beam pointing.

For the issues identified in these areas, participants considered what experiments could be conducted using the NIF to address or even resolve these issues. Participants identified what developments (e.g., in diagnostics, predictive capabilities, etc.) would be needed in parallel with NIF development, construction, and operations, to support full utilization of the NIF for such experiments. Participants were encouraged to suggest any improvements to the NIF design that would improve its capability in performing the proposed experiments.

The second plenary session on the last day provided summaries of each workshop session, discussion of the results, and follow up work to provide better definitions of proposed experiments and supporting requiirements for testing on NIF.

\subsubsection{General Findings of the NIF-IFE Workshop}

Relevance to IFE. NIF can uniquely provide a large and essential portion of developments needed for the IFE Program to more firmly establish the feasibility and technical basis for the design of an Engineering Test Facility (ETF), the major IFE facility to follow the demonstration of ignition in NIF. NIF was found to provide major opportunities in all the IFE areas covered: IFE target physics and design/performance optimization, IFE fusion chamber dynamics and first wall response, IFE fusion power technology, materials science and safety, and 
final precision-integration tests of massfabricated IFE targets and high-repetition target injection systems. Achievement of ignition in NIF will itself provide a major prerequisite for IFE.

The ignition target physics development activities for the ICF defense program will accomplish the most challenging target development requirements for IFE. Experiments that characterize materials response to target emissions (ablation, generation of shocks, chamber vacuum recovery) will be of importance to both defense and energy applications of ICF; the experiments will aid the development of design codes for both programs. Accomplishing the defense program on NIF will automatically develop many technologies and codes relevant to IFE and ETF design, which accentuates NIF's dual-benefit capability:

- Demonstration of fusion target ignition, propagating burn, and energy gain;

- System integration information provided by the design, construction, and operation of the NIF itself;

- Demonstration of first wall and optics protection schemes in the target chamber;

- Data on high-dose-rate neutron effects on materials;

- Data on neutron heating, tritium breeding reactions, activation, and shielding; and

- Development of cryogenic targets for NIF.

There will, however, also be many opportunities to use NIF for experiments and tests that are specific and important to IFE but not necessarily required for defense program needs:

- Design and optimization of capsules and hohlraums for nonlaser drivers such as heavy-ions;

- Tests of advanced targets that produce higher gain, allow simplified illumination geometry, can be massproduced at low cost (reduced precision requirements), and can survive highvelocity injection;

- Techniques for fast chamber vacuum recovery and behavior of protective liquid films and jets;
- Tests of single-shot breeding and neutron multiplication performance of small subscale IFE blanket modules or liquid jets;

- Experiments to measure tritium burn-up and recovery efficiency; and

- Tests of model IFE target-beam engagement precision and repeatability in a multishot chamber environment ( 5 $\mathrm{Hz}$, four-shot burst of injectable, thinwall hohlraum targets).

IFE Code Development and Benchmarking. The proposed uses of NIF for IFE development are aimed primarily at understanding the basic underlying target and target chamber phenomenology, physics, and materials science for IFE. There are far too many IFE concepts and technology choices to all be tested by trial and error, so using the NIF to establish predictive design capabilities is one of the keys to costeffective IFE development. The IFE design codes are needed to design the ETF and to guide the selection of technology developments needed for future IFE power plants. These same codes would also be used to design the Laboratory Microfusion Facility (LMF), a larger-yield ICF defense capability. (Section 6 discusses the possibility of LMF and ETF sharing a single driver.) Accordingly, the purposes of the NIF experiments are to benchmark and improve the computational tools to be used for future IFE system designs, instead of trying to pick winners and losers of IFE technology options from empirical tests. Therefore, it is sufficient that NIF provide target chamber environment conditions similar to, but not necessarily the same as, conditions expected in specific IFE power plant concepts.

NIF Shots Needed Specifically for IFE. Besides the initial shots for demonstrating ignition on the NIF, there are many additional shots following ignition that would be very important and specific to the IFE tests identified above. The number of additional shots was estimated to be in the range of $2000-3000$, including perhaps 1000 that would require yield, and 200 to 400 that would require high yield. These IFE-specific shots need to be coordinated with NIF shots for weapons physics, nuclear weapons effects tests (NWET), and high-energy 
density science. Many of the IFE experiments (e.g., for chamber dynamics, IFE fusion technology and materials tests) could probably be conducted concurrently with other non-IFE experiments, but this number is undetermined.

Laser-Generated $X$-ray and Debris Target Sources for IFE Experiments. Many experiments that study material responses to soft $x$ rays and debris, with ablation and shock formation, do not require targets that ignite. Some of the lasergenerated $x$-ray and debris sources for these IFE experiments would be the same as those discussed in the NWET laser-generated $x$-ray/debris sources. Some would be designed to generate more debris than for NWET targets. The NIF laser energy is large enough to provide characteristic soft $x$-ray and debris loadings of $>100 \mathrm{~J} / \mathrm{cm}^{2}$ over $>1000 \mathrm{~cm}^{2}$ samples, mimicking in-chamber IFE conditions. Nova experiments can achieve similar intensities, but not with samples large enough to support IFE-relevant spatial and time scales for the ablated material evolution. Laser energies of at least $400 \mathrm{~kJ}$ at $3 \omega$, with single-sided illumination geometry, may suffice for some of these types of

IFE experiments.

NIF Design Impacts. Nearly all of the potential IFE development experiments suggested for NIF could be performed within the present NIF laser system and target area design capabilities, and functional requirements. IFE development experiments would not require expensive modifications to the NIF laser or target chamber. The following possible design modifications or additions, however, could significantly enhance the utility of NIF for IFE development:

1. Diagnostics for post-bang-time characterization of target emissions including angular and energy distributions within the target chamber are required. These include fast, spatially resolved pressure transducers, neutron activation and heating microcalorimeters, ion mass spectrometers, and visible/UV/x-ray backlighters positioned to view target debris and wall ablation, including droplet motion, on relevant spatial scales. Many of these new diagnostics for specific IFE experiments would benefit from initial development tests on Nova before using them on NIF.

2 Several suggested IFE experiments involve various amounts of liquid and ablated solid test samples which would condense or solidify on the NIF chamber walls and optics windows. Compatibility tests are needed to find out what condensates the NIF Vacuum Recovery System (VRS) can clean up. The tests will also guide us in selecting compatible liquid and solid surrogates to model the dynamics of IFE material responses. These debris problems may be incompatible with the main NIF target chamber, or the problems may result in long chamber recovery times for cleanup. Therefore, these IFE experiments could benefit from the addition of a small ( 1 - to 2-m diam) test chamber using laser-generated $x$-ray/debris targets with $\sim 400 \mathrm{~kJ}$ of laser energy.

3. One or two larger ports of about 1- to 2-m diam for conical enclosures that can mount large IFE test assemblies and be inserted in reentrant geometry will be needed to test 1) blanket/first wall sub-modules and 2) isochoric heating of small liquid jets.

4. The present NIF design has flexibility to include direct-drive capability. This capability will significantly expand the range of IFE target experiments that can be tested.

5. To support the credibility of operating an IFE chamber at high-repetition rate (which may be needed for an ETF decision in 2005), it is highly desirable if NIF could shoot a sequence of two to four IFE model targets on the fly with reproducible laser illumination symmetry. Significant offline development of IFE model targets, the injection system, and tracking system would be needed first, but eventually a few multishot precision/ integration tests would be done in the main NIF target chamber. The idea is to test the accuracy of injected target-emplacement and alignment at the chamber center in the postshot chamber environment for several successive shots. The model IFE targets would not need to be ignited to provide this test. For example, the reproducibility and 
symmetry of NIF laser illuminations could be imaged through empty, thin-wall hohlraums or by diagnosing sub-ignition neutron yields. The NIF laser system would need the ability to stagger-fire four sections of the 192 beamlets about $200 \mathrm{~ms}$ apart, with appropriate front-end and flashlamp delays between the separate beamlet groups. If these target injection tests are needed to support the ETF decision, they must be ready shortly after first ignition is achieved ( 2004-05). This means that the development of the IFE model targets, the tracking system, and the target injection system need to start within a few years (i.e., by 1998).

\subsection{IFE Target Physics Experiments}

\subsubsection{Introduction}

In addition to the target physics experiments planned for the initial ignition campaign for ICF and defense applications, NIF can also perform experiments that explore target physics issues specific to IFE. The baseline target designs for the NIF are indirectly driven; this is, of course, reflected in the NIF design of the illumination system and the target chamber. (A means to convert the indirect-drive scheme to an acceptable direct-drive scheme, however, has been developed. This scheme may allow up to $1.5 \mathrm{MJ}$ of blue light on a direct-drive target. NIF advanced conceptual design activities will consider this option.) Although the workshop focused on indirect-drive targets and heavy ion driver, there is still substantial interest in directdrive targets and in laser and light-ion drivers for IFE. For this reason we considered direct- and indirect-drive targets with lasers, light ion, and heavy drivers.

A significant number of the target physics issues for IFE will be addressed by the planned experiments on the NIF for ignition and defense sciences. These include optimization of target yield and gain, studies of capsule implosion characteristics, and studies of capsule ignition and burn physics. In parallel with this effort, theoretical models and simulation codes must be developed and benchmarked. In fact, the benchmarking of ICF computer codes in a regime of parameter space similar to that of IFE may be one of the most significant contributions of the NIF to IFE. Thus, the planned NIF experiments will, by themselves, greatly benefit IFE. The NIF should also perform additional experiments important to IFE, which may not necessarily be part of current planning. Completely addressing IFE ion target issues on NIF will rely on the design of and experimentation with ion-like laser targets, such as the ones shown in Fig. 3.2-1.

Table 3.2-1 shows that the NIF can address and largely resolve most IFE target physics issues. The NIF, along with Omega Upgrade, PBFA II, and other ICF facilities around the world, will be able to address all of the issues. The completion of these experiments will provide the target physics basis to proceed with an ETF. Indirect-drive IFE target experiments require only minor changes to the NIF conceptual design, if any. Furthermore, the target diagnostics and codes developed for the core program are directly applicable to IFE targets. Conducting experiments where consistent and symmetric illumination of a target could be obtained using only a fraction (e.g., 1/4) of the beams would allow a series of such targets (e.g., four) to be fired in quick succession by stagger-firing different groups of beams. Since each of the 192 beamlets of the NIF is independent of others in terms of spectral and temporal characteristics, this type of experiment is routinely possible.

\subsubsection{Variety of Pulse Shapes}

Some IFE capsule concepts may require pulse shapes that differ substantially from the baseline NIF capsules. Thus, it would be desirable if the NIF could be designed with some flexibility in its pulse shaping capabilities.

\subsubsection{Radiation Flow, Illumination Geometry, and Internal Pulse Shaping}

As noted above, ion drivers are the primary candidate for IFE, therefore, laser driver ion 
Heavy ion-like laser targets
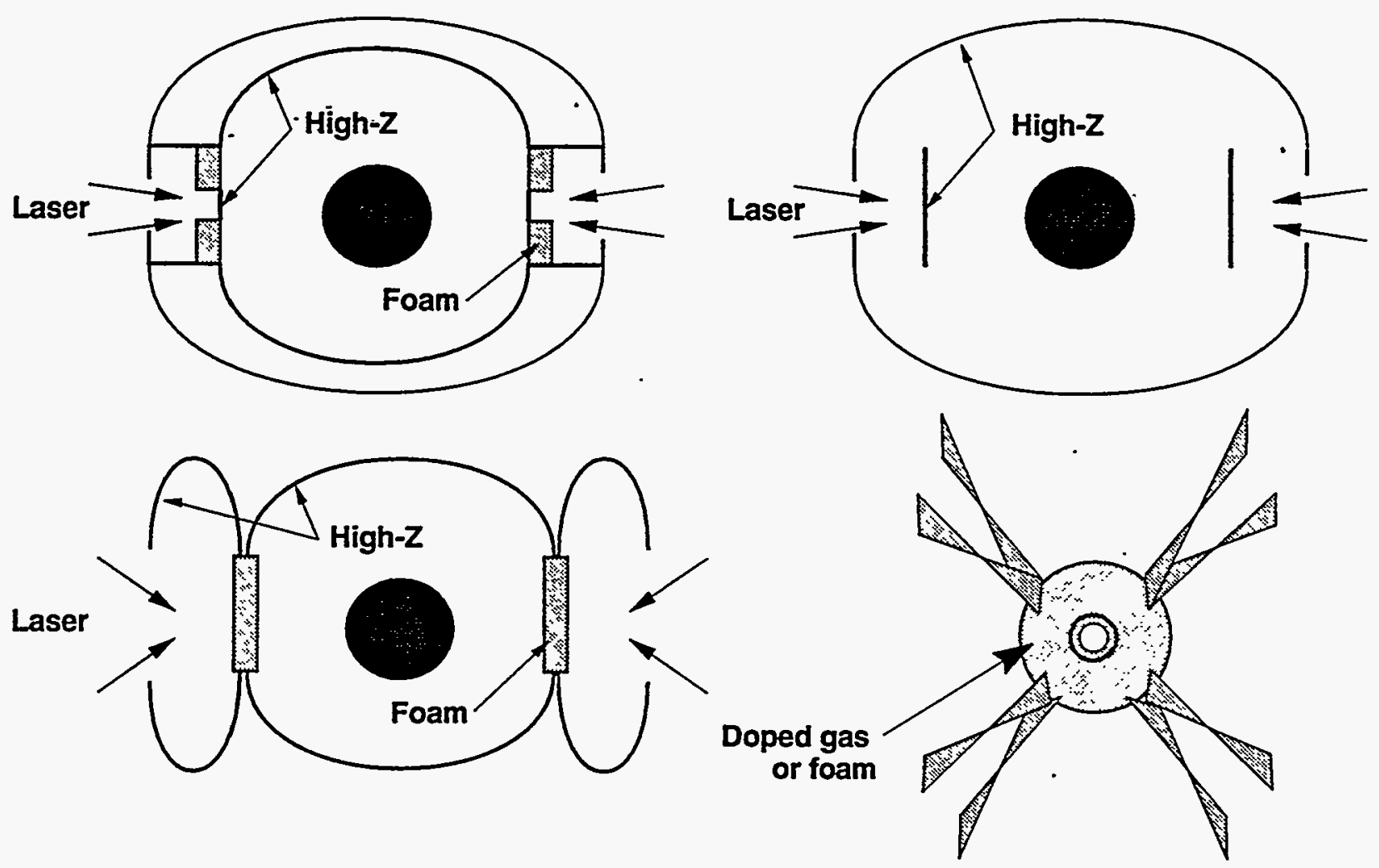

70-37-0195-0180pb01

Figure 3.2-1. Schematic of ion-like laser targets.

Table 3.2-1. IFE target physics issues for ion and laser drivers, direct and indirect drive. An ' $\mathrm{X}$ ' indicates issues that can be largely resolved with the NIF capabilities.

\begin{tabular}{lcccc}
\hline & \multicolumn{2}{c}{ Ion drivers } & \multicolumn{2}{c}{ Laser drivers } \\
& Indirect & Direct & Indirect & Direct \\
\hline \hline Usability of a variety of pulse shapes & $\mathrm{X}$ & $\mathrm{X}$ & $\mathrm{X}$ & $\mathrm{X}$ \\
Radiation flow, illumination geometry, and & $\mathrm{X}$ & & $\mathrm{X}$ & $\mathrm{X}^{*}$ \\
internal pulse shaping & & & & \\
Sensitivity of capsules to radiation asymmetry & $\mathrm{X}$ & & $\mathrm{X}$ & $\mathrm{X}$ \\
Materials issues (capsule, hohlraum, ablator) & $\mathrm{X}$ & $\mathrm{X}$ & $\mathrm{X}$ & $\mathrm{X}$ \\
Fabrication surface finish and precision & $\mathrm{X}$ & $\mathrm{X}$ & $\mathrm{X}$ & $\mathrm{X}$ \\
Capsule mounting and injection & $\mathrm{X}$ & & $\mathrm{X}$ & $\mathrm{X}$ \\
Power vs energy tradeoffs & $\mathrm{X}$ & & $\mathrm{X}$ & $\mathrm{X}$ \\
Output spectra and shielding & $\mathrm{X}$ & $\mathrm{X}$ & $\mathrm{X}$ & $\mathrm{X}$ \\
Reduced tritium use & $\mathrm{X}$ & $\mathrm{X}$ & $\mathrm{X}$ & $\mathrm{X}$ \\
Advanced targets & $\mathrm{X}$ & $\mathrm{X}$ & $\mathrm{X}$ & $\mathrm{X}$ \\
\hline
\end{tabular}

- Due to direct-drive capability of NIF. 
targets, such as those shown in Fig. 3.2-1, are of interest. Furthermore, other advanced target concepts considerably different from the standard NIF laser targets are of interest. One of the important issues is what can we learn about these nonstandard targets from the NIF. Here we focus on accurate radiation flow experiments, and, in particular, symmetry issues. One of the primary limitations on IFE target gain is the achievable density-radius product of the fuel which can be limited by the drive asymmetry. If techniques can be developed to improve implosion symmetry, then higher gains might be achieved, or restrictions on beam illumination geometry may be reduced.

The NIF is a laser facility designed to be flexible. The specific techniques it may test to achieve drive symmetry could be adapted to IFE drivers. Our goal on NIF is to explore symmetry issues that are appropriate to high gain with IFE drivers. For example, achieving good radiation drive symmetry with single-sided beam illumination would show the credibility of this illumination geometry, which simplifies the driver-target chamber interface.

A specific experiment would use the NIF beams to produce $x$ rays in a geometry that simulates the radiation pattern created by indirect-drive ion beams. In this way we can study radiation flow and symmetrization techniques that closely resemble those used in IFE targets. These techniques would include bumthrough barriers and opaque shields. Such experiments could begin on Nova, but would need the NIF to study the impact on ignition and burn. It will be important to understand the relation between instability growth, mix, and asymmetry in order to predict IFE target performance.

Several diagnostic techniques are available to study symmetry. The reemission ball technique can be used to study early-time symmetry. The late-time symmetry can use witness ball and symmetry-capsule methods. All three of these diagnostic techniques can be used on the NIF, and they have considerable development from Nova experiments.

Radiation Flow in Low-Density Hohlraum Fill Materials. Some high-gain ion-driven hohlraum concepts involve the use of a lowdensity foam fill for converting ion energy into $x$ rays; they ensure that early-time ion beam imprinting asymmetries do not degrade the capsule drive symmetry. Experiments that address this design issue would involve measurements of the smoothing capabilities of low-density foam to purposely impressed asymmetries in planar and, eventually, spherical geometries.

Internal Pulse Shaping IFE Target Concepts. High gain at low-absorbed energy will require a precise control of the shock waves to compress the fuel along a nearly Fermi-degenerate adiabat. For the baseline NIF ignition target, this shock control is entirely provided by a precise timeshaping of the laser power input (beam pulse shaping). Some ion-driven target designs involve concepts where the shock history is controlled, at least partially, through time-dependent radiation flow and ablator modification in the hohlraum and/or capsule (internal pulse shaping). Such schemes may be of interest for IFE because driver complexity and cost might be reduced by relaxing the degree of ion beam pulse shaping and, thereby reducing bunching and focusing schemes required for achieving high gain.

Experiments that address internal pulse shaping concepts involve converting a square laser pulse into multiple shocks in an internal pulse shaping package at first in planar geometry and eventually in spherical implosion geometries.

\subsubsection{Sensitivity of Capsules to Radiation Asymmetry}

The experiments just described are concerned with producing and diagnosing particular radiation environments. A closely related issue is determining the sensitivity of capsules to asymmetries. Determining how to modify the capsule shape to compensate for asymmetries in the radiation field is also valuable. By varying the hohlraum length and the irradiance in the inner and outer NIF beams, the radiation flux on the capsule can be tuned to achieve known asymmetries (e.g., Legendre modes 2 and 4 , and other modes). The capsule geometry would be modified to achieve a nearly spherical shape at 
peak compression. Capsule performance and imaging could determine the limiting physics. Substantial fusion yield could be used to diagnose degradation by instability growth and mix.

As noted earlier, if single-sided illumination can be used in an IFE target, driver and fusion chamber design may be simplified. An important objective of these experiments is to learn if capsules can tolerate the odd Legendre modes created by single-side illumination.

One issue for a fusion chamber is the number of the required penetrations of the chamber wall and their locations. A goal is to minimize the penetrations and allow large regions of chamber wall with no beam penetration. This is a particular concern for direct drive, where nearly uniform distribution of the beams is likely, and no substantial region of solid angle is unpenetrated. Typical experiments would test techniques to control low-order (Legendre modes less than about 10) asymmetry with a reduced number and location of beams. This might be done by adjusting the laser beam profile and power history.

\subsubsection{Materials Issues}

An experimental target physics study is needed to investigate a variety of target materials. Many of the materials variations of interest to IFE will be studied as part of the defense programs applications, including varying the material composition of the hohlraum to improve the laser absorption and $x$-ray conversion efficiency in order to improve target performance, and optimizing the capsule materials to improve the capsule implosion efficiency or tailor the $x$-ray output spectra for particular experiments.

Experiments for resolving target physics issues or for optimizing target performance unique to IFE have been defined. One key difference between IFE and defense programs ICF is the repetition rate of target shots. This difference leads to a significantly greater concern of toxicity and activation for IFE; these concerns are reasons for varying the target materials for IFE. One key experiment would be to determine the target performance variation when the hohlraum material is changed from the typical gold to lead. Lead has slightly different radiation transport properties, and we want to experimentally verify that these changes do not significantly impact target performance. Another possible modification is to vary the capsule materials to decrease toxicity of the capsules in the reactor and in the fabrication process. These experiments are relatively straight-forward in that these targets could be fabricated and fielded on the NIF. Target performance (yield, stability, etc., as compared to standard NIF targets), as a function of design, would be measured.

\subsubsection{Target Fabrication Surface Finish and Precision}

Determining the target surface finish requirement and precision, and uniformity of the various layers of the target will be a part of the defense programs effort on the NIF. However, certain surface finish and precision issues will arise from the IFE requirement for mass production of targets. The target performance tradeoffs with surface finish and precision need to be quantified for IFE. (This is discussed further in Section 3.4, Target Systems.) No NIF modifications from the current design will be needed for these experiments.

\subsubsection{Capsule Mounting and Injection}

A target physics issue unique to IFE arises because IFE targets must be injected at high velocity $(100 \mathrm{~m} / \mathrm{s}$ or $\mathrm{so})$. To achieve that velocity, the target must be accelerated (by gas gun, etc.). The capsule in an indirect-drive target must be held in the center of the hohlraum by a structure (typically a pair of mylar sheets) that is strong (thick) enough to support the capsule without breaking or without excessive vibration, and does not significantly perturb the implosion. A study of the target performance variation with the thickness of the support structure is needed to provide information to IFE target fabrication specialists. It should be noted that this is currently a difficult calculation to perform with existing codes, and experimental verification of results is considered essential to resolve this issue. 
In addition; it may be desirable to support the capsule in alternate geometries so it can withstand acceleration. These alternate geometries can also be experimentally examined.

\subsubsection{Power vś Energy Tradeoffs}

For some ion beam IFE driver concepts, there may be cost tradeoffs involving peak power vs energy delivered. In particular, energy delivered at a lower peak power will generally be less costly per unit energy. Thus, an exploration of relatively low-drive temperature ablator materials (e.g., Be or doped LiH), high-aspect ratio capsules, and a thorough mapping of the hydrodynamic instability limit of power-energy space for ignition on NIF, will be useful information for IFE target designers.

\subsubsection{Output Spectra and Shielding}

Experiments that address how to modify the of emission of charged particles, neutrons, and $x$ rays from imploded targets, will give insight into how a NIF target could evolve into an IFE target. Material ablated from the target chamber wall is determined by time, energy, and angular dependent spectra of the above emissions. The beam transport (particle beams or laser beams) could also be affected by the details of the target emission.

Charged Particles (Debris). The particles emitted from an indirect-drive target system can be tailored by changing the thickness (mass) of the hohlraum and the distribution of the mass in the hohlraum wall. Experiments could be done to measure the charged particle spectrum in energy, time, and angular distribution for a variety of hohlraum designs. Preliminary experiments that could be done on NOVA globally would address the emission from the hohlraum and could be used to develop the charged particle spectrometer needed to do the measurement. The energy spectrum is particularly important because it will indicate the degree of ion implanting we can expect from the charged particle emission.

Neutrons. Neutron moderators could be used to shift the neutron energy spectrum to lower energies. Existing diagnostic techniques are adequate to measure the modifications to the neutron energy spectrum. Preliminary experiments could be done on either Nova or the Omega Upgrade to determine the amount of moderator needed for the experiments.

$X$ rays. All of the diagnostics needed for the $x$-ray measurements will be available in the base set of NIF diagnostics. The filtering of the $x$ rays could be measured as a series of shots that are "ride along" shots to the main NIF ignition experiments. Dedicated shots could study the modification to the $x$-ray spectrum and angular distribution by changing the mass distribution of the hohlraum wall.

\subsubsection{Reduced Tritium Use}

Reduced tritium loadings in the capsule will reduce the tritium throughput through the target factory loop, thereby reducing the radiological hazards. The major issue involves checking the ranges of fusion products and in-flight reactions with nonequimolar DT. These issues are fairly well understood. The effect on yield of in-flight DD reactions is at most a factor of two. Integral experiments checking the variation of yield with tritium loading should be performed on NIF.

\subsubsection{Advanced Targets}

There may be qualitatively different inertial fusion targets which offer major advantages for inertial fusion energy.

The Fast Ignitor. This new concept, described in Ref. 1, separates the implosion phase from the ignition phase of the inertial fusion process. First, the fuel is compressed via a conventional implosion, being driven by lasers or ion beams either directly or indirectly. After the high-density core is formed, the capsule corona is driven out of the way with the ponderomotive pressure of a somewhat higher intensity laser beam. Finally, an ignition beam, with intensity approximately $10^{20} \mathrm{~W} / \mathrm{cm}^{2}$ is incident on the capsule, where the light is converted to relativistic electrons. The electrons propagate from critical density to the peak capsule density, where they heat the fuel to ignition temperature.

There are several advantages to this approach. First, the threshold of high gain $(<100)$ is sharply reduced to approximately $100 \mathrm{~kJ}$ from the 5-10 MJ of conventional designs. Second, the 
peak gain is approximately an order of magnitude higher than in the conventional approach. The extra gain could be traded against other system advantages: lower tritium loadings in cryogenic fuel layers ờr noncryogenic fuel where the thermonuclear fuel is chemically bound in the pusher. Third, the approach works with reduced uniformity requirement; the fast ignitor also works with much lower peak-density fuels, allowing the peak intensity supplied by the implosion drivers to be much lower. Finally, the fabrication quality of the implosion may also be reduced.

The Fast Ignitor is a speculative idea. Initial research will be performed on Nova. High-gain applications await NIF to assemble large fuel masses with adequate symmetry and an ignitor laser beam with approximately $20 \mathrm{~kJ}$ of energy at peak intensity. There should be adequate space in the space frame to support the additional laser beams and ports to inject the beams into the chamber. This option should be available in both direct-drive and indirect-drive chambers.

\subsubsection{Number of Shots}

We made estimates of the number of shots that might be required to resolve the various issues (see Table 3.2-2). The uncertainties in the estimates are large. Also, as explained in the introduction, some of these experiments may be performed as part of the defense program. Moreover, a selection of driver and target type would reduce the required number. Finally', some of the experiments might be deferred to the engineering test facility.

\subsection{Target Chamber Dynamics Experiments}

\subsubsection{Introduction}

Many of the basic issues of IFE identified in past fusion power plant conceptual design studies that relate to target chamber dynamics can be addressed on the NIF. The basic IFE issues that we relate to chamber dynamics are listed in Table 3.3-1. The four basic categories are: 1) IFE target emissions, 2) material response, 3) gas. dynamics, and 4) miscellaneous. The target emissions category covers issues related to the characteristics of IFE target emissions in radiation and debris. Material response covers issues of

Table 3.2-2. The number of low, medium, and high yield shots required to address the issues and the utility of other facilities.

\begin{tabular}{|c|c|c|c|c|}
\hline - & $\begin{array}{l}\text { Low } \\
\text { yield }\end{array}$ & $\begin{array}{l}\text { Medium } \\
\text { yield }\end{array}$ & $\begin{array}{l}\text { High } \\
\text { yield }\end{array}$ & $\begin{array}{l}\text { Utility of } \\
\text { other } \\
\text { facilities }^{a}\end{array}$ \\
\hline Usability of a variety of pulse shapes & some & some & 20 & \\
\hline $\begin{array}{l}\text { Radiation flow, illumination geometry, } \\
\text { and internal pulse shaping }\end{array}$ & 100 & & & +++ \\
\hline Sensitivity of capsules to radiation asymmetry & 700 & 200 & 50 & + \\
\hline Materials issues (capsule, hohlraum, ablator) & some & some & 20 & $++t$ \\
\hline Fabrication surface finish and precision & 100 & 50 & 10 & ++ \\
\hline Capsule mounting and injection & 30 & 15 & 5 & +++ \\
\hline Power vs energy tradeoffs & 700 & 200 & 50 & $+t+$ \\
\hline Output spectra and shielding & \multicolumn{4}{|c|}{ Mostly add-ons } \\
\hline Reduced tritium use & & & 20 & \\
\hline Advanced targets & \multicolumn{4}{|c|}{ Hundreds } \\
\hline
\end{tabular}

a The number of $+s$ indicates the utility of other facilities. 
Table 3.3-1. Summary of IFE issues in Target Chamber Dynamics session.

\section{Target response \\ $\mathrm{X}$-ray spectra (inside and transmitted) \\ Debris generation \\ Hohlraum material design \\ Capsule design effects on debris \\ Neutron generation \\ Anisotropy of all emissions \\ Mass transport}

\section{Material response}

\section{A. First surfaces}

Equations of state

Ablation(melting and vaporization)

Condensation

Shock loading

Fracture (solids)

Spallation

Pitting and cratering

Splash and break up (liquids)

Net erosion of wall

Particulates (droplets)

Plasma debris (hohlraum)

Physical, property degradation by neutrons

Chemical property degradation by neutrons

Surface chemistry

Anisotropic effect ( $x$-rays and debris)

Mass distribution of debris

Hazards to final driver optics

Cumulative damage

\section{B. Blanket (if not first surface)}

Isochoric heating

Cavitation/water hammer
III. Gas dynamics

A. Chamber clearing/condensation

Dissociation/recombination/equations of state

Chemical kinetics

Radiation transport

Sticking efficiency and surface chemistry

Role of noncondensables

Vaporization, condensation

Droplet control

Mass transport

Venting through structures

Vacuum requirements

B. Beam propagation

Ion beam transport loss

Laser beam propagation SRS loss

Emissions impact on driver optics

Gas-fill pressure effects

Cavity clearing

Condensation on cryo target

IV. Miscellaneous

$5 \mathrm{~Hz}$ IFE operations and chamber clearing

Cost of energy conversion (MHD)

EMP radiation effects (including debris) at the first surface of the structural wall or blanket. Gas dynamics includes issues of chamber clearing, condensation, and beam propagation. The miscellaneous category includes such issues like multiple-pulse radiation effects.
Preparatory experiments on Nova and other facilities are key for assisting in the development of predictive capabilities for chamber dynamics. A NIF target with a yield of $20 \mathrm{MJ}$ gives approximately the same fluence of soft $x$ rays and target debris on surfaces $\sim 1 \mathrm{~m}$ away as a 
350-MJ yield target gives to walls $\sim 4 \mathrm{~m}$ away in an IFE power plant target chamber. There are also several experiments that could be performed using the laser only with energies of $100-400 \mathrm{~kJ}$. A NIF operational issue may be the diagnosing and handling of liquids used in neutron isochoric heating experiments. Table 3.3-2 contains some experiments needed to address these issues.

\subsubsection{Target Response}

Target $x$ rays and debris drive many target chamber phenomena and must be well understood. Verification of computer codes used to simulate the emissions during disassembly of targets will be important to the design of an IFE power plant. Though the targets in NIF will not be exactly the same as in an IFE power plant, they will serve as a good basis on which to do this verification.

The NIF is unique in its ability to provide the proper conditions for these experiments. Some information could be obtained from Nova target experiments if the diagnostics to measure the time and direction-dependent spectra are employed. However, significant thermonuclear yield will only be obtained on NIF, which is an important test of the computer codes. Since the target experiments will be performed, there are no additional requirements for the NIF facility except more diagnostics will be needed. We

Table 3.3-2. Materials response experiments on the NIF.

\begin{tabular}{|c|c|c|c|c|c|c|}
\hline $\begin{array}{c}\text { Issues } \\
\text { addressed } \\
\end{array}$ & Experiment & Purpose & $\begin{array}{l}\text { Ignition } \\
\text { required? }\end{array}$ & $\begin{array}{c}\text { Special } \\
\text { diagnostics } \\
\text { required }\end{array}$ & $\begin{array}{c}\text { Use } \\
\text { second } \\
\text { chamber? }\end{array}$ & $\begin{array}{c}\text { NIF } \\
\text { modifications }\end{array}$ \\
\hline Ablation & $\begin{array}{l}\text { Ablation } \\
\text { issues }\end{array}$ & $\begin{array}{l}\text { Bench-mark } \\
\text { ablation in } \\
\text { codes }\end{array}$ & Desired & $\begin{array}{l}\text { Pressure } \\
\text { transducers* } \\
\text { and mass } \\
\text { spectrometer }\end{array}$ & Yes & $\begin{array}{l}\text { None } \\
\text { (extra } \\
\text { material near } \\
\text { target) }\end{array}$ \\
\hline $\begin{array}{l}\text { Erosion } \\
\text { (ablation, } \\
\text { melting, } \\
\text { surface } \\
\text { chemistry) }\end{array}$ & $\begin{array}{l}\text { Response of } \\
\text { materials to } \\
\text { single/ } \\
\text { multiple } \\
\text { pulses }\end{array}$ & $\begin{array}{l}\text { Measure } \\
\text { response of } \\
\text { surface } \\
\text { materials }\end{array}$ & Desired & None & Yes & $\begin{array}{l}\text { None } \\
\text { (some impact } \\
\text { from vapor- } \\
\text { ized or } \\
\text { spalled } \\
\text { material) } \\
\end{array}$ \\
\hline $\begin{array}{l}\text { Isochoric } \\
\text { heating, } \\
\text { cavitation, } \\
\text { splash of } \\
\text { liquids }\end{array}$ & $\begin{array}{l}\text { Liquid } \\
\text { responses }\end{array}$ & $\begin{array}{l}\text { Study } \\
\text { isochoric } \\
\text { heating, fluid } \\
\text { mechanics }\end{array}$ & Yes & $\begin{array}{l}\text { Pressure } \\
\text { transducers } \\
\text { and strain } \\
\text { gauges }\end{array}$ & No & $\begin{array}{l}\text { Prepare for } \\
\text { liquid metals, } \\
\text { Si oils, etc. \& } \\
\text { graphite } \\
\text { placed near } \\
\text { target }\end{array}$ \\
\hline $\begin{array}{l}\text { Ablation } \\
\text { impulse, } \\
\text { pressure } \\
\text { loading, stress } \\
\text { parameters }\end{array}$ & Wall stresses & $\begin{array}{l}\text { Bench-mark } \\
\text { stress codes }\end{array}$ & Yes & $\begin{array}{l}\text { Pressure } \\
\text { transducers* }\end{array}$ & No & $\begin{array}{l}\text { None } \\
\text { (vapor } \\
\text { condensation) }\end{array}$ \\
\hline Activation & $\begin{array}{l}\text { Pulsed } \\
\text { Neutron } \\
\text { Activation }\end{array}$ & $\begin{array}{l}\text { Study pulsed } \\
\text { neutron } \\
\text { effects }\end{array}$ & Yes & None & No & None \\
\hline
\end{tabular}

* Fast-response and high-sensitivity transducers.

recommend target experiments to develop capabilities to control the following: 1) the

partition between $x$-ray and debris energy, and

2) the spatial distribution of the energy. Some 
additional NIF requirements may be required for these experiments.

The $x$-ray diagnostics may not require additional development, while the debris measurement will likely sequire additional development. For the $x$ rays, time-dependent $x$-ray spectrometers will be positioned to observe $x$ rays coming 1 ) from the capsule directly out the laser entrance holes, 2) coming from the inner surface of the hohlraum case out the laser holes, and 3) through the hohlraum case. The debris needs to be measured in the same directions. Some diagnostics for measuring the debris (ion energies and species, ion flux vs time, shrapnel size and velocity) need to be developed. For IFE power plants, shrapnel may not be an issue and the development of shrapnel diagnostics is of lower priority than ion diagnostics. Computer codes that simulate $x$-ray and debris generation will also be developed. In fact, these experiments will greatly aid in code development.

\subsubsection{Material Response}

Material response is fundamental to target chamber dynamics. This is indicated especially by the many first-surface issues shown in Table 3.3-1. Most of these issues deal with the characterization of first surface ablation, erosion, and other net changes in surface materials, including cumulative damage. The experiments recommended in Table 3.3-2 (except for neutron activation) are based on these issues. We expect that all of the proposed NIF experiments for IFE concerning material response will require ignition, because this environment is so similar to IFE conditions (including neutron effects) than could otherwise be provided. Most of the proposed NIF experiments for IFE concerning material response can be performed within the existing NIF specifications. But an ion mass spectrometer is needed, and some diagnostic development is required to obtain fast-response high-sensitivity pressure transducers.

Preparatory $x$-ray response experiments for some of these effects can be performed on Nova. It would also be desirable to perform ablation and erosion experiments in a second NIF chamber where neutrons are excluded, and where radiation fluence levels would be higher than in Nova but lower than in the NIF.

To address the liquid responses of curtains and jets specific to particular fusion-chamber designs, the NIF must deal with the possibility of liquid ejecta and vapors throughout the chamber. These experiments may be significantly more expensive than other proposed experiments because of the preparation and cleanup required. Additional experiments for measuring hoop stresses and stress concentrations, and pulsed neutron activation of wall materials should be performed concurrently as part of general NIF operations. All of these experiments will serve to benchmark codes for extension of predictive capabilities to IFE conditions.

\subsubsection{Gas Dynamics}

Key Issues. Gas dynamics plays an important role in the transport of materials ablated by $x$-rays and target debris, momentum transfer to liquids and structures, and condensation. Gas dynamics is important to chamber clearing, along with other mechanisms such as the sweeping action of "fresh" injected liquid jets. Modeling of gas dynamics and condensation for IFE must address several fundamental topics: dissociation and recombination, equations of state, chemical kinetics, radiation transport, sticking efficiency and surface chemistry, role of noncondensables, vaporization, condensation, droplet control, venting through structures, ion beam transport loss (stimulated Raman scattering loss for laser beams), and vacuum requirements.

Needed Experiments. Considerable information that validates chamber gas dynamics codes can be obtained from shock tube experiments, including shock propagation through complex geometries. ${ }^{2}$ For condensation phenomena, experimental information is also available, however, only for conditions (temperatures, vapor velocities, and time scales) well outside the range of interest for IFE. The transport and condensation of ablated materials in IFE chambers involve tremendous variation in the material chemical and thermodynamic states (densities from solid/liquid to gas to vacuum, temperatures from $500^{\circ} \mathrm{C}$ to several $\mathrm{eV}$ ). 
Experiments will be required to validate equations of state, radiation transport models and assumptions, and multidimensional hydrodynamic models. Condensation at surfaces, required to attenuate struictural loading and to restore chamber vacuum, will involve chemical kinetics, mass transport with multiple chemical species including noncondensables, sticking efficiency and surface chemistry. Additional experiments will be required to address these areas, experiments requiring capabilities that will only be available with the NIF facility.

Experiments are currently underway on the Nova facility to provide initial verification of design codes. However, fluences on Nova are too small to provide sufficiently large pressure signals $(>10,000 \mathrm{~Pa}$ ) with sufficiently long time scales ( $>20 \mathrm{~ms}$ ) for experimental resolution of shock propagation. The NIF facility is therefore required to benchmark the suite of codes, which will be employed for IFE chamber clearing design. Experiments addressing condensation phenomena will involve significantly different time scales. Thus, a number of different experiments will be required to address the issues listed above. The specific experiments proposed by the workshop are:

Ablation, gas dynamics, and condensation experiments. As discussed earlier, experiments using small cylindrical chambers are currently underway on the Nova facility. $X$ rays enter through a small opening at one end of the chamber, ablate copper at the base of the cylinder, and generate a shock front.

Condensation of ablated materials is measured by post-test chemical analysis, while pressure transducers measure propagation of the shock. The relatively low power of the Nova facility requires a short stand-off distance for the ablated surface, giving a small chamber dimension and a short time scale for shock propagation, only marginally within instrument resolution. A larger experiment fielded on NIF will give detailed, useful benchmarking data.

Condensation effects. This series of experiments will focus on condensation on surfaces. Initial experiments will study onedimensional (1-D) condensation of ablated material on a plane surface to validate fundamental models; later experiments will examine condensation on droplets to study multidimensional effects where condensation occurs while shocks propagate through coolant spray. The experiments are important for validating models for condensation of hot vapors on relatively cold surfaces (e.g.; chamber designs using liquid jet blankets). For the 1-D experiments, available $x$-ray diagnostics would be used. For the droplet spray experiment, droplets will be collected to measure energy gain using calorimetry.

We recognize that many of the experiments that have been carried out, and the codes that have been developed and validated as part of the Nuclear Weapons Effects Tests (NWET) program may be relevant to IFE. This body of knowledge, if it could be made available, would be exceedingly useful to IFE.

First wall condensation experiment. These experiments will attach plates of various materials to the NIF chamber wall; it will use the NIF wall itself to investigate condensation of hot vapors on a hot surface. The experiments are aimed at studying phenomena related to the net erosion of material, as well as the sticking of vapor atoms and surface chemistry. Pressure transducers may be employed to measure material dynamic response.

Performance evaluation of the cavity during self clearing. These experiments focus on chamber clearing issues for wetted wall chambers. The primary issue is clearing time, which determines repetition rates for these designs. Laser diagnostics for vapor density and temperature measurements will be employed.

Facility Requirements. The experiments described above can be performed in the NIF facility without major modification. Because the gas dynamics and condensation phenomena are generated by $x$-ray and debris energy, fusion yield is not required for the all of the experiments. Yield is, however, desirable for some experiments, and is required for the first wall condensation experiment. The primary concern is that these experiments inherently involve ablation of significant quantities of materials. Some of the materials will potentially deposit on the debris shields, thereby decreasing 
the number of possible shots before maintenance is required. The possibility of adding a second, smaller chamber run from one bank of beamlets to provide a test facility where ablation and contamination with IFE materials would be more acceptable should be seriously investigated.

Developments Required. Improvements and development of instrumentation capabilities are desirable for these experiments; however, modest extensions to existing instrumentation are adequate for obtaining useful experimental information. Thus, these experiments can be performed at modest cost. It would be desirable, though not critical, to have a second smaller chamber available for gas dynamics and condensation experiments where constraints on optics contamination could be relaxed (with a smaller number of debris shields to be cleaned and with simpler access without NIF activation levels).

\subsubsection{Beam Propagation}

Ion drivers lead the list of preferred drivers for IFE. NIF should be used, as much as possible, to assess potential IFE scenarios for ion beam final transport and for focusing in the reactor chamber. This includes both HIF and LIF scenarios. Experiments fall into four areas as listed in Table 3.3-3.

1) Experiments that assess the effects of the various fusion yield source terms ( $x$-rays, neutrons, ions, debris, shrapnel, etc.) on the final focusing elements. For LIF scenarios that use a solenoidal focus lens, experiments should be performed on NIF to assess the following: lens shield material erosion, effects on the lens current-carrying system, and erosion effects on the central lens plug. For HIF ballistic transport scenarios, the effects of the various source terms on the final focusing quadrupole triplet and its shielding should be assessed.

2) Many ion beam transport scenarios use a background gas in the reactor chamber.

Pressures may be from $10^{-3}$ to $\sim 10$ torr. Gases at the higher pressures and/or higher atomic number $(\mathrm{Z})$ can be effective in absorbing $x$ rays from the fusion yield. However, higher pressures and higher $Z$ lead to increased scattering and emittance growth of the beam. NIF could be used to help assess the optimum pressure for these scenarios. This information would be valuable for both HIF and LIF, for ballistic transport in the 1 torr regime, and for free-standing channel transport in the 1-10 torr regime. Specifically, gas-fill pressure experiments could examine:

i) X-ray absorption and fireball effects at $\sim 1-10$ Torr that protect the first wall

ii) Materials protection effects for ion beam optics or laser beam optics

iii) Gas degradation effects on cryogenic targets, delivered laser energy (including stimulated Raman scattering), and NIF diagnostics.

3) All ion beams may have transport losses that depend on the gas pressure and the transport scenarios. These beam transport issues cannot be addressed on NIF because they require ion beams. Several codes (IPROP, IVORY, QUICKSILVER, BUCKSHOT, etc.) can be used to address these issues. These codes will be benchmarked with experiments on ion beam facilities.

4) All ion beam transport scenarios for IFE require that the chamber be cleared after each fusion shot, and that the appropriate conditions for efficient ion beam transport be reestablished again before the next shot. NIF could be used to assess several of these cavity clearing issues for both HIF and LIF transport scenarios.

These experiments could be staged progressively from Nova, to a second chamber on NIF, to the main NIF chamber, to high-yield NIF. No substantial modifications to the NIF baseline design are required for these experiments. The effects of extra material being deposited on the debris shields in NIF must be considered. New diagnostic development is required to adequately diagnose the gas/plasma conditions in these experiments. 
Table 3.3-3. Beam propagation experiments on the NIF.

\begin{tabular}{|c|c|c|c|c|c|c|}
\hline Issue & Experiments & Nova & $\begin{array}{c}\text { NIF } \\
\text { second } c \\
\text { hamber }\end{array}$ & NIF & $\begin{array}{c}\text { High- } \\
\text { yield } \\
\text { required? }\end{array}$ & $\begin{array}{c}\text { New } \\
\text { developments }\end{array}$ \\
\hline $\begin{array}{l}\text { Ion driver IFE } \\
\text { final transport } \\
\text { and focus optics } \\
\text { degradation }\end{array}$ & $\begin{array}{l}\text { 1) Täget yield effects on HIF } \\
\text { solenoidal lens (less shield, } \\
\text { lens winding, annular } \\
\text { beam plug) } \\
\text { 2) Target yield effects on HIF } \\
\text { final focusing optics } \\
\text { (quadrupole triplet) }\end{array}$ & $x$ & $x$ & $\mathrm{x}$ & $x$ & . \\
\hline $\begin{array}{l}\text { Gas-fill pressure } \\
\text { effects for IFE }\end{array}$ & $\begin{array}{l}\text { 1) X-ray and debris } \\
\text { absorption in a fill gas } \\
\text { (consider pressures of } 10^{-2} \\
\text { to } 10 \text { torr) Specifically, } \\
\text { examine: } \\
\text { - X-ray absorption and } \\
\text { fireball effects at } \sim 1-10 \\
\text { torr to protect the first } \\
\text { wall } \\
\text { - Materials protection } \\
\text { effects (ion beam optics } \\
\text { or laser beam optics) } \\
\text { - Gas degradation effects } \\
\text { for a) cryo targets, } \\
\text { b) reduction of } \\
\text { delivered laser energy } \\
\text { (including stimulated } \\
\text { Raman scattering), and } \\
\text { c) NIF diagnostics }\end{array}$ & & $x$ & $x$ & $x$ & $\begin{array}{l}\text { Diagnostics } \\
\text { and codes }\end{array}$ \\
\hline $\begin{array}{l}\text { Ion beam } \\
\text { transport losses }\end{array}$ & 1) Cannot be done on NIF & & & & & \\
\hline $\begin{array}{l}\text { Cavity clearing } \\
\text { for IFE }\end{array}$ & $\begin{array}{l}\text { 1) Time to reestablish } \\
\text { quiescent background } \\
\text { conditions for next shot }\end{array}$ & & & $x$ & $x$ & $\begin{array}{l}\text { Diagnostics } \\
\text { and codes }\end{array}$ \\
\hline
\end{tabular}

\subsubsection{Miscellaneous}

Key IFE Issues. Several additional issues can be addressed on NIF:

1) The vulnerability of electronics used for diagnostic sensing and control of an IFE fusion chamber to target EMP generation needs to be measured and modeled with predictive codes.
2) IFE success should not depend solely on one driver concept; an affordable development of a potentially efficient "rep-ratable" driver alternatives to the mainline heavy ion approach is needed for a robust driver development program.

3) The credibility of $\sim 5 \mathrm{~Hz}$ IFE operation cannot be completely established by single-shot experiments alone.

4) IFE development cost is justified only if IFE offers a competitive advantage; lower cost 
energy conversion unique to IFE is needed to address the dominant balance-of-plant cost.

Needed Experiments. EMP measurement and modeling, tests of compact, multipass regenerative laser amplifier chains and optics damage, and tests of target plasma energy conversion at high-power density are needed. For rep-rate operation, the changes in debris and $x$-ray ablation of first wall surfaces caused by surface morphology and temperature gradients from previous shots under hi-rep rate conditions needs to be determined; chamber dynamics code benchmarks need to be extended from single shots to at least multishot bursts at high rep-rate.

Use of NIF. The above IFE issues could be addressed on NIF through the suggested EMP, Laser Driver, High Rep-Rate Chamber Dynamics, and MHD experiments listed in Table 3.3-4. The Laser Driver demonstration is based on the single-shot NIF design; it does not address diode pumping and component cooling issues needed for an IFE laser driver.

NIF Use Justification. All these issues could be addressed in new separate facilities. Since NIF can be used to address these proposals with little or no modification or additions of equipment, it is cost effective to perform these experiments in NIF. EMP and Laser Driver demonstration can be performed concurrently with other NIF experiments. MHD may need dedicated shots with LiH target shells. All these proposals should cost less than a few million dollars in specific capital/operating. High-Rep Rate Chamber Dynamics would need to use all 1.8 MJ of NIF beams stagger fired in a burst; this would be very expensive to duplicate in any separate facility.

NIF Facility Requirements. No new facility requirements are needed for EMP and Laser
Driver. Addition of trace oxygen to the NIF vacuum recovery system may be needed for LIH condensate cleanup for the MHD Conversion experiment. A 4-to 12-shot repeating injector for foil targets would be needed for the High RepRate Chamber Dynamics experiment. A . basketball-size model rep-rate chamber would need to be inserted into the NIF target chamber for this latter experiment.

NIF Baseline Modifications. No modifications are required to the NIF baseline design for these experiments, except possibly for sound-vibration damping modifications for the beam transport optic mounts in the High RepRate Chamber Dynamics experiment. In-chamber EMP diagnostics would be added to the NIF Phase I diagnostic set in the EMP experiment. No additional diagnostics are needed for Laser Driver or MHD, except for probes built into the MHD generator channel; the probes are integral with that item inserted in a manipulator.

\subsubsection{Preparation for NIF Experiments}

The activities required in preparation for conducting IFE experiments on NIF include development of new diagnostics, use of Nova, use of ion beam facilities, consideration of a second smaller NIF chamber, and code development.

New Diagnostics. There is a need for devices to measure the ion velocities (energies), species, and flux originating from target source debris. Also, there is a need for modest extensions of existing instrumentation in experiments related to gas dynamics and condensation phenomena. Moreover, there is a

Table 3.3-4. Miscellaneous NIF experiments.

\begin{tabular}{llcc}
\multicolumn{1}{c}{ IFE issue } & \multicolumn{1}{c}{ Experiment } & Nova & NIF fusion \\
pre-exp? & yield? \\
\hline \hline Electronic survivability & EMP & No & Yes \\
Alternate IFE driver & Laser Driver Demonstration & No & Yes \\
$\sim 5 \mathrm{~Hz}$ IFE operation & High Rep-Rate Chamber & No & No \\
Energy conversion cost & MHD Conversion & No & Yes \\
\hline
\end{tabular}


need for diagnostic development to adequately diagnose the gas/plasma conditions in potential beam propagation scenarios.

In addition to specific diagnostics, there is a general need for diagroștic protection against electromagnetic interference, especially from the high-yield environment arising from gammainduced EMP. Here, the IFE community can benefit from the NWET community, which has already attempted measurements in highradiation environments.

Use of Nova. Experiments should be performed on Nova to further characterize emitted $x$-ray and debris characteristics (including anisotropy) from target foils made of materials considered for IFE targets. Experiments onx-ray responses of first-surface ablation, erosion, and some other surface effects (e.g., cumulative $x$-ray and debris damage and multiple-shot debris effects) should also be performed on Nova. Additionally, preparatory experiments should be performed to examine $x$-ray and debris effects on ion-beam final focus magnet materials.

Use of Ion Beam Facilities. Experiments on ion beam facilities are needed to benchmark codes for ion beam transport losses that depend on gas pressure and on transport conditions that will be present in NIF.

Development of a Second Smaller NIF Chamber. A smaller chamber may be cost effective for gas dynamics and condensation experiments, where only a few hundred kilojoules of laser energy is needed. The anticipated heavier debris-shield contamination could be tolerated and be spared the activation associated with the main NIF chamber. Work could be done on $x$-ray responses of first surface ablation, erosion, and multiple-shot surface effects such as cumulative $x$-ray and debris damage, and cumulative heating effects. Partial simulations of a multiple-shot environment for gas dynamics could be studied by having at least two debris sources (e.g., hohlraum and target inserter, with other possible inserters). Further preparatory experiments could be performed on the smaller NIF chamber to investigate $x$-ray and debris effects on ion-beam optics materials, and on gas-fill pressure.

Code Development. Although there are many useful codes for estimating NIF behavior, many of these will require additional physics or modifications to meet IFE needs. There are three classes of codes that require development:

1) multiphase (solid, melt, vapor) hydrodynamic, 2) gas hydrodynamic including condensation and some chemical effects, and 3) ion beam codes. Table 3.3-5 shows examples of a few such codes and the developments needed. In addition, some ion beam codes will need benchmarking.

Table 3.3-5. Example IFE chamber codes.

\begin{tabular}{|c|c|c|}
\hline Code & Type & New developments \\
\hline CONRAD 6 & $\begin{array}{l}\text { 1-D radiation-hydro, ablation physics } \\
\text { (no } \mathrm{T}-\mathrm{N} \text { burn) line transport }\end{array}$ & Laser deposition, 2-D version \\
\hline HYADES 7 & $\begin{array}{l}\text { 1-D radiation-hydro } \\
\text { (T-N burn) laser deposition }\end{array}$ & $\begin{array}{l}\text { Ion deposition, low energy density physics, } \\
2-D \text { version }\end{array}$ \\
\hline SRIPUFF 8 & 1-D hydro (fracture, no T-n burn) & $\begin{array}{l}\text { Integrate with HYADES for radiation } \\
\text { transport Cumulative damage models }\end{array}$ \\
\hline L2D9 & 2-D hydro (fracture, no T-n burn) & $\begin{array}{l}\text { Integrate with 2-D HYADES for radiation } \\
\text { transport }\end{array}$ \\
\hline PHD $4^{9}$ & $\begin{array}{l}\text { 1-D radiation-hydro } \\
\text { (T-N bum, ion deposition) }\end{array}$ & Laser deposition \\
\hline TSUNAMI $^{1}$ & 2-D hydro, simple ablation boundaries & Radiation transport, boundary conditions \\
\hline
\end{tabular}


Many of the basic target chamber dynamics issues of IFE identified from past IFE power plant conceptual design studies ${ }^{3-5}$ that can be addressed on the NIF are: 1) characterization of IFE target soft $x$-ray and-debris emissions, 2 ) the response of first wall materials and protective wall fluids to those target emissions, and 3) the subsequent gas dynamics of the vapor blow-off in chamber clearing, vapor condensation, and vacuum recovery. Compared to Nova, NIF has a larger laser energy than NOVA to test ablation and shock response of material samples large enough to exhibit time and spatial scales more relevant to IFE at energy fluxes representative in a power plant. A typical NIF target output at a full target yield of $20 \mathrm{MJ}$ will put $\sim 48 \mathrm{~J} / \mathrm{cm}^{2}$ of soft $x$-rays and target debris plasma on surfaces $\sim 1 \mathrm{~m}$ away from the target; this fluence is similar to the deposition from $350 \mathrm{MJ}$ yields on walls $\sim 4 \mathrm{~m}$ away in an IFE power plant target chamber. Thus, the NIF can provide a reduced-scale test chamber environment representative of an IFE power plant, for a limited number of shots. CONRAD, ${ }^{6}$ HYADES, ${ }^{7}$ SRIPUFF8, ${ }^{8}$ L2D, ${ }^{9}$ PHD $4,{ }^{9}$ and TSUNAMI $^{1}$ are a number of $1-D$ and 2-D hydrodynamics and radiation hydrodynamics codes that could be calibrated and improved with NIF chamber dynamics data. To acquire the data needed for these codes, NIF chamber dynamics experiments will need new diagnostics to measure the ion velocities (energies), species, and flux originating from targets; NIF also needs modest improvements of existing instruments to measure gas dynamics and condensation phenomena (e.g., fast response pressure transducers).

Figure 3.3-1 is an example of a possible NIF experiment designed to test the predictions of a chamber dynamics code such as TSUNAMI. The test assembly consists of a conical chamber in which a test material at the back surface is ablated by $x$ rays that are admitted through a hole in the larger front plate. The conical shape will provide a better test of the 2-D modeling capability of the codes than would a cylindrical chamber. The high fluences attainable on NIF will permit using a relatively long chamber (for longer time-scale gas dynamics), while still providing a large amount of ablated vapor from the rear surface. Passage of the expanding vapor is recorded by pressure transducers placed along the length of the cone. This information will enable us to confirm the predicted velocities and shock reflection strengths (from the plate at the large end of the cone) of the model. The experiment will also allow is to estimate condensation rates to be obtained from the reduction in pressures at later times. Distribution of condensed material will be determined from post-shot analysis of the cone's inner surface. Examination of the ablated disk after the shot will determine the amount of material ablated, an important input parameter for the gas dynamics calculations.

\subsection{Fusion Power Technology}

\subsubsection{Introduction}

Fusion power technology (FPT) in IFE includes components whose primary functions are energy conversion, tritium production and processing, and radiation shielding. These components include the first wall and its protection mechanism, the blanket, vacuum vessel, shield, and tritium processing systems. Also relevant to FPT are remote maintenance systems and those elements of targets and drivers that provide either the source term and/or

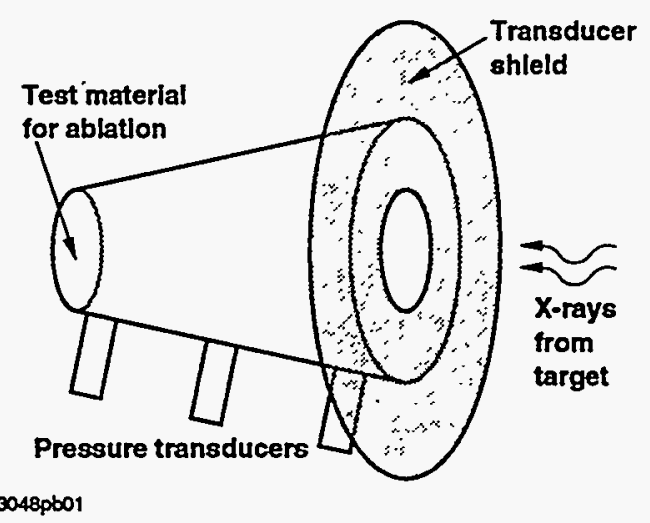

Figure 3.3-1. Example of a possible NIF experiment designed to test the predictions of a chamber dynamics code such as TSUNAMI. 
have their performance significantly affected by the radiation environment.

The dominant issues for FPT in IFE power plants concern the nuclear and material performance of components to achieve economic competitiveness and to realize safety and environmental advantages. NIF will provide valuable information to IFE-FPT, both from the demonstrated performance and operation of the basic facility itself and from data obtained from experiments designed specifically to test FPT issues.

NIF's relevance to FPT are both in its prototypical size and configuration, and in its prototypical radiation field (neutrons, $x$ rays, debris) spectra and intensity per shot. For example, the neutron fluence from a 20-MJ NIF target at $1-\mathrm{m}\left(5.7 \times 10^{13} \mathrm{n} / \mathrm{cm}^{2}\right)$ is similar to that from a 350-MJ IFE target at 4-m $\left(6.2 \times 10^{13} \mathrm{n} / \mathrm{cm}^{2}\right)$. These features allow the possibility of conducting a range of valuable FPTrelated experiments on NIF. The most important limitation of NIF for these FPT experiments is the low repetition-rate. The planned repetition rate in NIF is too low to produce time-averaged relevant bulk nuclear heating and reactions. The consequences of the NIF features and limitations are clear; experiments that can give information in a single shot on NIF are generally relevant and useful, but experiments that require many successive (back-to-back) pulses are generally not meaningful on NIF.

\subsubsection{Overall Contribution of NIF to IFE/FPT}

Analysis of FPT issues and the range of possible experiments on NIF suggest that the most important contributions of NIF to FPT development for IFE are the following:

1) Achieving ignition

2) System integration (i.e., design, construction, and operation of the NIF facility will provide important information on the interactions among many IFE subsystems including FPT omponents)

3) Testing of first-wall protection schemes

4) Obtaining data on dose rate effects on radiation damage in materials
5) Obtaining data relevant to tritium self sufficiency in IFE power plants (e.g., tritium burn-up fraction in target, some tritium inventory and flow rate parameters, and data on achievable tritium breeding rate),

6) Neutronics data on radioactivity, nuclear heating, and radiation shielding. Efforts and deliberations by the workshop participants suggested specific FPT experiments on NIF that appear to be both viable and useful. Future efforts will be needed to address the details of such experiments and their impact on the NIF design and its operation. Below, specific examples of the utility of NIF to address a number of FPT-related issues are summarized.

\subsubsection{Material Damage}

One area of concern to IFE is radiation effects. NIF is unique in that it provides a very high dose rate in each shot, equivalent to about 10 to 1000 displacements per atom per second (dpa/s). However, the low rep-rate limits the dose to a maximum of about $10^{-4} \mathrm{dpa}$ in one year. Therefore, NIF will not produce a design database for IFE in metal alloys and composites (e.g. swelling, creep, embrittlement, Young's modulus, and thermal conductivity). However, NIF will be useful for basic physics of radiation effects in materials. Examples include:

a) cascades (morphology, size, fraction of free and clustered defects, impurities), b) microstructural evaluation, c) electrical properties, d) optical properties (fiber optics, coatings), and e) molecular cross linking. NIF can provide data essential to test Molecular Dynamic Simulation (MDS) codes, ${ }^{10}$ leading to a capability to predict microscopic bulk material responses to neutron damage. Figure 3.4-1 illustrates how MDS calculations that predict overlap of damage clusters (a dose rate effect). This can be compared with a sample exposed to a single NIF shot.

The incentive to produce low-activation hazard structural materials for IFE also creates a dilemma. While the neutron activation characteristics may be fairly well understood, their mechanical properties under IFE conditions are generally unknown. The impact on the mechanical properties of materials compared to 


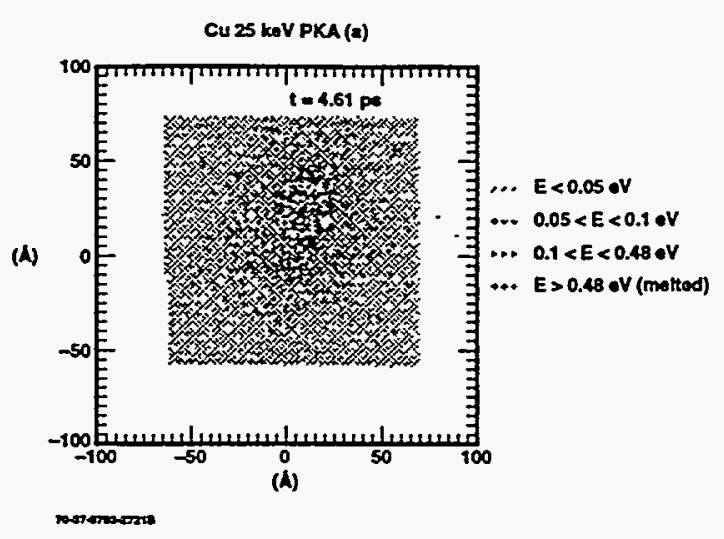

Figure 3.4-1. MDS predictions can be validated with observations using MDS results like this.

continuous irradiation (such as in MFE) is not known. In addition, this short-pulse neutron effect must be understood in conjunction with other effects such as $\mathrm{H}, \mathrm{He}$, and transmutation production, high-cycle fatigue, and thermal cycling, all at in-reactor temperatures.

In some low-activation materials like $\mathrm{SiC}$, we predict that for IFE, a large number of damage sites are produced in each pulse. Interstitials from one damage site combine with the vacancies created not only at that site, but also from adjoining or nearby sites, such that there is an increased rate of recombination compared to the steady state situation. Interstitials that do not find sinks in vacancies create clusters together, which increases clustering over the steady state example. Increased recombination tends to reduce void swelling, while increased clusters tends to increase embrittlement.

A predictive capability that calculates material responses to IFE irradiation would be a powerful IFE design tool. A candidate for part of this capability is MDS. This is a deterministic approach to predicting damage; MDS calculates responses at an atomic level by quantifying the response of a three-dimensional (3-D) array of atoms to knock-on atoms that impinge on the matrix from a range of angles, and with a range of energies as a result of an incident neutron flux. Potentially, MDS capabilities may include predicting the number of vacancies and interstitials that will result from neutron irradiation, as well as the cluster fraction of defects, atomic mixing and solute precipitation, and predict phase transformations. Its accuracy is dependent on the accurate modeling of interatomic potentials.

An overall material property predictive capability would use the results of MDS predictions along with a Monte Carlo defect annealing treatment; couple this 'source term' to firite element codes, and then predict the changes in bulk mechanical properties (e.g., ultimate strength, Young's modulus, and fatigue lirnits). Data to benchmark MDS is essential if it is to be used as part of such a predictive capability. $\mathrm{NiF}$ can provide a source of relevant data for this purpose.

On NIF, neutrons will be produced two ways: 1) from exploding pusher (directly driven) experiments that are frequently used to calibrate neutron diagnostics, and 2) from indirect-drive targets, with ignition. (Neutrons will be produced from NIF nonignition indirect-drive shots, but the neutron yield of these, at best, will likely be about the same as the direct-drive shots). With 1.8-MJ laser energy and a larger exploding pusher target, NIF will produce $\sim 10^{15}$ neutrons. Samples to be exposed to this neutron fluence could potentially be placed within $1-2 \mathrm{~cm}$ of the target, provided shrapnel generation was tolerable. Such experiments would be possible as soon as the NIF laser is activated ( 2001). A series of experiments could be conducted to produce neutron damage in very small, thin samples. The first samples chosen for exposure would be those that have been well characterized, and are most computationally scrutinized by MDS, such as Ag, $\mathrm{Cu}$, and $\mathrm{Fe}$. The damaged samples would be examined with electron microscopic techniques to determine such damage parameters as the number, distribution, and sizes of defect clusters (see Fig. 3.4-2). These measurements would be compared to MDS predictions. Table 3.4-1 shows that NIF would create more than enough damage sites to allow confident validation of MDS techniques. 


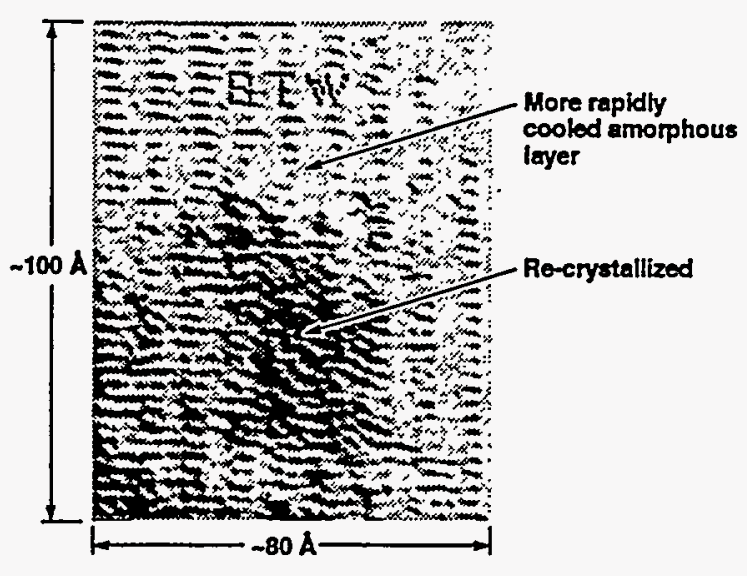

70-37-0793-2722ppb01

Figure 3.4-2. High resolution electron microscope image of damage site in high temperature superconductor (Y-Ba-Cu oxide).

Another area of importance to material behavior is thermomechanical effects. NIF is unique in that it provides IFE prototypical thermal surface loads by $x$ rays and debris, in the range of $10^{-1}$ to $10^{-3} \mathrm{~J} / \mathrm{cm}^{2}$. Therefore, a limited amount of information for design and code verification can be obtained for IFE. Examples include: a) mechanics of bonded structures (composite interphase interfaces, mirror coatings, protective coatings), b) materials evaporation/ ablation response, c) surface damage (flaking, spalling, material loss, shrapnel), and d) thermoelastic wave bulk damage (fatigue, fracture).

\subsubsection{Wall Protection}

A large number of wall protection concepts have been proposed for IFE power designs, covering a diverse range of concepts with different testing needs. There are many issues associated with wall protection. They can be classified in three main categories:

1) Blast effects on film protectant thickness and stability. In thin film schemes, control of the film thickness is required over large surface areas which are exposed to different amounts of $x$-ray, debris, and vapor shock loadings. If the liquid surface disassembles (for example by ejecting droplets or creating aerosols), then cavity clearing may become impossible on a time scale consistent with reactor repetition rates. Both thick and thin films are subject to various types of disassembly due to high-intensity energy deposition and instabilities. In addition to these "surface forces," pulsed volumetric isochoric heating causes forces which can disassemble liquid films and jets. Experiments are needed under reactor-prototypical loading conditions to demonstrate adequate control over protectant film thickness and to observe any liquid ejection and/or major disruptions to film surface.

2) Film/substrate interactions due to blast impulse. In some thin film designs, a porous (either rigid or flexible) substrate holds and/or feeds the film protectant. This porous substrate is expected to have a stabilizing effect on the film, preventing droplet ejection or other effects listed

Table 3.4-2 NIF creates more than enough damage sites for MDS.

\begin{tabular}{ccccc}
\hline Material & $\begin{array}{c}<\mathrm{T}> \\
\left(\mathbf{E}>\mathbf{E}_{\mathbf{d}}\right) \\
(\mathbf{k e V})\end{array}$ & $\begin{array}{c}\sigma_{\text {int }} \\
\text { (barns/neutron) }\end{array}$ & $\begin{array}{c}\text { Displacements per } \\
\text { atom (dpa) } \\
\left(\times \mathbf{1 0}_{-9}\right)\end{array}$ & $\begin{array}{c}\text { Number of NIF } \\
\text { neutron damage } \\
\text { sites* }\end{array}$ \\
\hline \hline $\mathrm{Si}$ & 500 & 1.8 & 4.8 & 180 \\
$\mathrm{C}$ & 689 & 1.2 & 0.8 & 270 \\
$\mathrm{SiC}$ & 600 & 1.5 & 3.0 & 150 \\
$\mathrm{Fe}$ & 228 & 2.5 & 4.6 & 425 \\
$\mathrm{Cu}$ & 190 & 2.85 & 4.7 & 500 \\
$\mathrm{Ag}$ & 72 & 4.1 & 2.5 & 500 \\
\hline \# of damage sites $=\sigma_{\text {int }} \times \phi \times \mathrm{V} \times \rho ; \phi=2 \times 10^{14} \mathrm{n} / \mathrm{cm}^{2} ; \mathrm{V}=10 \mathrm{~mm}^{2} \times 2000 \AA=2 \times 10^{-11} \mathrm{~cm}^{3}$
\end{tabular}


above. In addition, the ability of the film to move through the substrate can attenuate the effects of the blast on the underlying structures.

3) Damage rates at dry spots on film-protected surfaces. The effects described above may lead to dry spot formation on the solid materials behind the protectant. Extended operation with dry spots is probably not tolerable. Experiments are needed to characterize damage rates at localized dry spots, including effects such as vapor shielding from the surrounding covered areas. The damage to dry spots should be characterized and the possibility of self-correcting of dry spots should be explored.

Many aspects of the issues above can be addressed in separate small-scale facilities. For example, basic film flows, film wetting and compatibility of the film and substrate do not require NIF. However, as described above, experiments and model development are needed to characterize the effects of the blast on these issues. A pulsed, high-power $x$-ray source would be useful to simulate surface loading from the blast. Simulation of volumetric loading also would be very useful, although no adequate facilities have been identified.

For wall protection issues, NIF provides unique capabilities for producing prototypical environmental conditions as compared with other available devices. For example, power plant relevant energy densities are achievable near the target. The use of prototypical targets also results in power plant relevant reaction product composition and spectra, and in prototypical instantaneous power density near target. This allows a unique capability to test simultaneous, synergistic effects at reactor-relevant loadings.

The overall cavity geometry is large and similar to a fusion power reactor, although this advantage is lessened to some extent by nonprototypicality of components, and by operating conditions within the cavity. The space available is large enough to test sizable ( $\sim 2 \mathrm{~m}$ length) elements of reactor components. Space becomes less available closer to the target due to beamline interference, but many important tests can be performed at 1-m or larger radius.
One of the most severe restrictions for component testing is the lack of continuous pulsing. Many important component issues depend on building up a high-average power density and/or require a large total number of pulses. Other limitations are related to the * surrounding geometry and/or environmental conditions. The cavity wall is expected to operate at low temperature, which will have a major impact on any issues related to cavity vapor transport and recondensation. Tests involving this important class of issues may require extensive modification to the cavity to properly simulate a reactor environment. The large number of laser beams ( 192) restrict the size of possible test articles, particularly close to target. For example, at a radius of $20 \mathrm{~cm}$ from the target, the maximum linear dimension will be $3-4 \mathrm{~cm}$. Additional interference with the tests will be introduced by the target holder apparatus, target diagnostics, and other equipment placed within the cavity during the tests.

The types of tests that can be performed in NIF include those used to verify code predictions and those aimed at providing direct experimental data for concept screening and validation. Examples of test geometries on NIF are shown in the figures below. For some tests, the $x$-ray yield is adequate at distances of $1 \mathrm{~m}$ or more from the target. At this radial location, space constraints are not critical. Figure 3.4-3 is an example of a film protectant experiment located within a 10-inch manipulator (TIM) for easy access to the chamber. Many protection schemes require larger dimensions, particularly in the vertical direction. These schemes may require specialized port access and support systems within the chamber. For protection experiments in general, liquids will be directly exposed to the vacuum chamber. These include liquid metals (such as $\mathrm{Li}, \mathrm{Pb}$, or $\mathrm{LiPb}$ ), Flibe, or simulant materials. Restrictions on exposure due to chamber contamination need to be clarified.

Prior to performing tests in NIF, a large amount of $R \& D$ will be needed to derive the maximum benefit from testing. Several types of development are needed: 

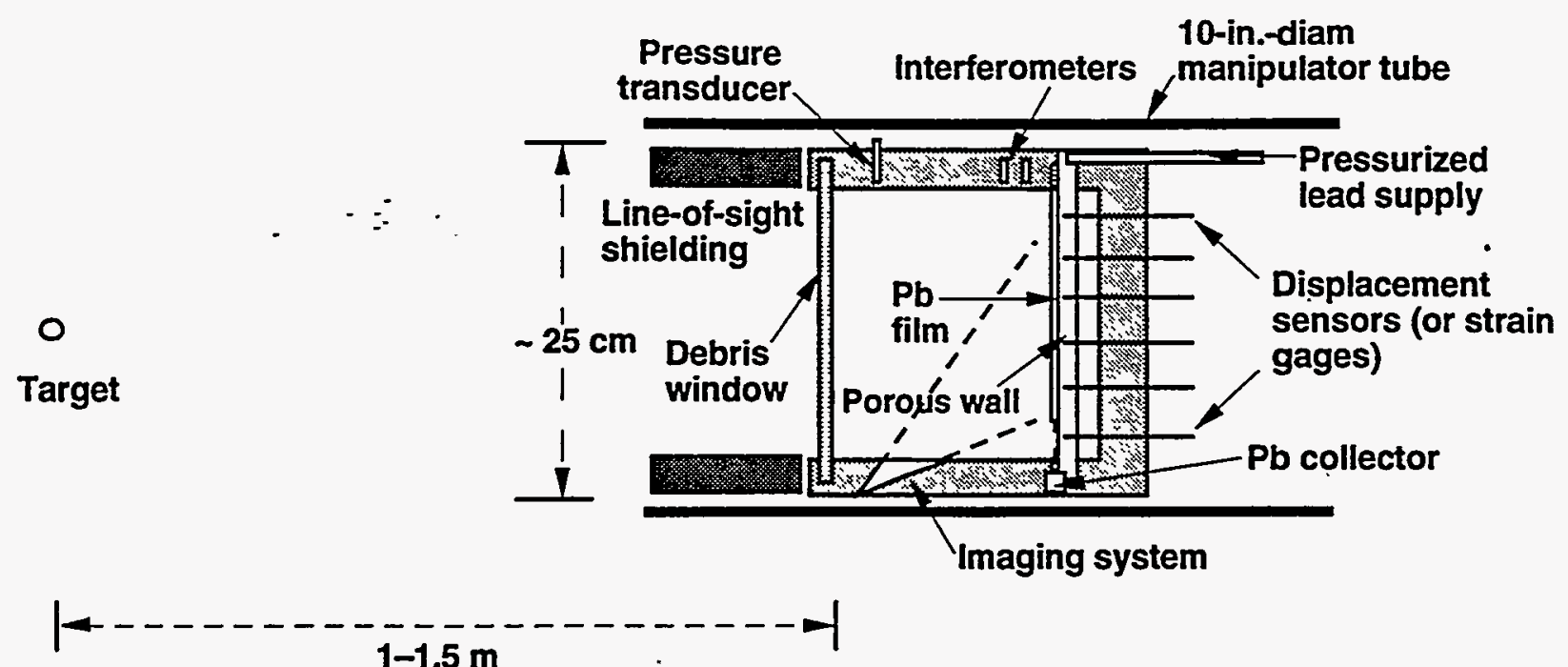

70-37-0185-0251pb01

Figure. 3.4-3. Thin film experimental setup.

1) Diagnostics. Numerous diagnostics are being developed for NIF to diagnose the target responses and energy yields from the blast. A large number of "engineering" diagnostics will be required to obtain useful information from testing. Some examples of needed measurements include: a) film shape, thickness and motion; b) surface temperature and density of vapor leaving surface; $c$ ) net impulse and deflection of panels;

d) mechanical response in structures;

e) cavity vapor temperature, pressure and composition; and $f$ characteristics of aerosols. While many of these diagnostics are well developed for conventional applications, special effort will be required to demonstrate their effectiveness in the NIF environment and/or develop new instruments, which can survive the blast.

2) Model development. A number of useful models currently exist. However, these tend to be simplified, often 1-D, approximations. Many of the key issues involve more complex phenomena resulting from nonuniformities or complex interactions. Further model development will be needed to interpret the results of tests, and to provide validation of codes which are sophisticated enough to be useful for design purposes.

3) Basic properties. In some cases, measurements of basic physical and chemical properties are needed to provide inputs to codes, and to interpret the results of tests.

4) Small-scale experiments. A large fraction of the remaining issues for wall protection can be addressed in existing or new facilities, which are cheaper or more useful than NIF. For example, basic film flow measurements are very important as a reference point for measurements in NIF, which include the effects of the blast.

\subsubsection{Radioactivity and Nuclear Heating}

A number of important neutronics experiments relevant to IFE appear to be viable on NIF, particularly for radioactivity/decay heat, biological dose and nuclear heating. These experiments include: a) radioactivity measurements in samples of materials to measure both short and long half-life isotopic activities; b) measurements of radioactivity from activated debris and shrapnel; c) measurement of biological dose outside the cavity and outside the building; and d) measurements of nuclear heating rate in 
samples of materials using microcalorimeter techniques. These measurements will be used to do the following: a) examine effects of high dose rate on double or triple neutron-induced reactions and transmutations; $b$ ) verify calculation codes and nuclear data libraries; and c) provide experimental data for licensing future facilities such as ETF.

Figure 3.4-4 shows the possible locations for measurements of radioactivity, biological dose and nuclear heating in NIF. Note that the average neutron fluence at the first wall $(\sim 5 \mathrm{~m}$ from the target) obtainable from an NIF target with a fusion energy yield of $20 \mathrm{MJ}$ is $\sim 2 \times 10^{12} \mathrm{n} / \mathrm{cm}^{2}$ per shot. Measurements have been conducted successfully recently at the Rotating Target Neutron Source (RTNS) facility in Japan and in
Tokamak Fusion Test Reactor (TFTR) with DT shots at comparable fluences.

Figure 3.4-5 shows a typical foil packet for radioactivity measurements. The experimental requirements for the radioactivity measurements include the following: a) fusion yield with fusion energy per shot $>0.1 \mathrm{MJ}$ (higher yields are better); b) support for 10 samples, each $\sim 10 \mathrm{~g}$ located $\sim 20 \mathrm{~cm}$ from the target; c) protection of samples from heating or damage; d) protection of samples from radioactive debris; and e) fast withdrawal ( $<10$ minutes) of irradiated samples following the shot.

Figure 3.4-6. shows a simplified picture of the microcalorimetric technique for measurements of . nuclear heating. The state of the art of present techniques is such that temperature rise greater

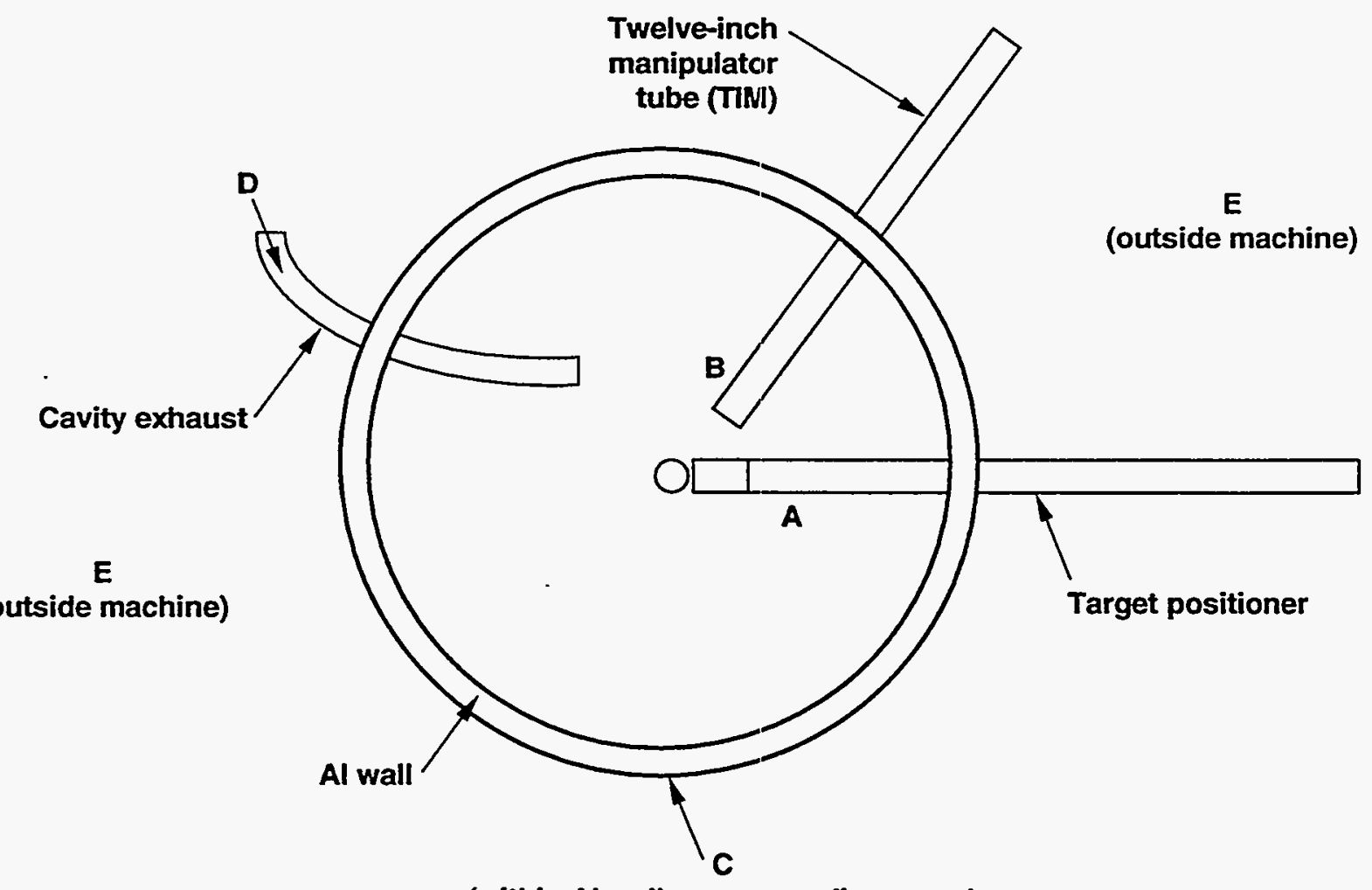

(within Al wall or surrounding areas)

Radioactivity and dose measurements at: A, B, C, D, E Nuclear heating measurements at: A/B, C

70-37-0195-0003p601

Figure 3.4-4. Locations of radioactivity, biological dose, and nuclear heating measurements for NIF. 


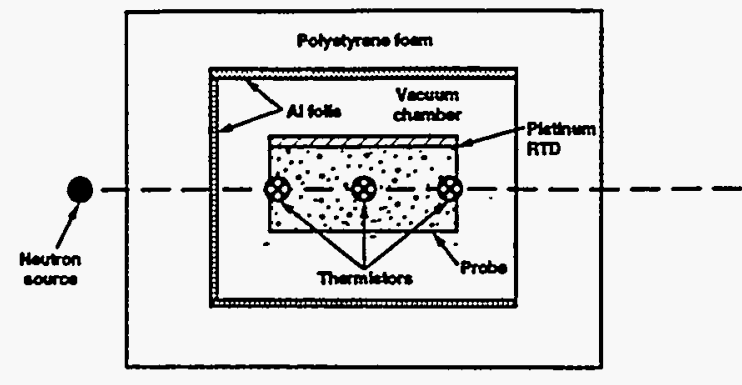

70-37-0195-0004pb01

Figure 3.4-5. A typical foil packet for irradiation.

than $0.001 \mathrm{~K}$ can be measured. Such a temperature rise is obtainable in NIF at $\sim 0.2 \mathrm{~m}$ from the target with a few MJ fusion yield. Higher yields will improve the accuracy of the nuclear heating measurements. Other experimental requirements to measure nuclear heating in NIF include a) support 5 to 10 calorimeters (each $\sim 800 \mathrm{~g}$ ) at symmetric locations, $b$ ) protection against non-neutron heating, c) protection against physical damage, d) extraction within 5 to 10 minutes after shot.

\subsubsection{Tritium Self Sufficiency}

NIF can make partial but important contributions to demonstrating tritium self sufficiency by performing the following measurements: a) those related to the required tritium breeding ratio (e.g. tritium fractional burn-up in the target, tritium flow rates, and tritium inventories wherever it is possible in the facility), and b) the potentially achievable tritium breeding ratio in prototypical blanket modules.

\subsubsection{Radiation Bulk and Penetration Shield}

The leakage of neutrons out of the target chamber could cause significant activation and radiation damage in out-of-chamber components of experimental, demonstration, and commercial reactors that will follow the NIF. The two primary components to the leaking neutrons are: 1) deep penetration through the chamber wall, blanket (when it exists) and shield, and

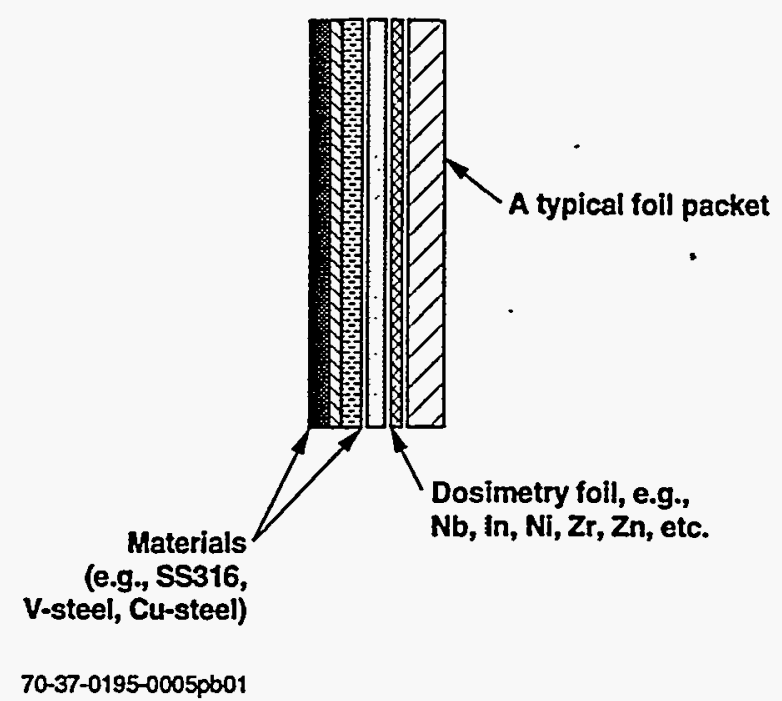

Figure 3.4-6. Simplified picture of a singleprobe calorimeter.

2) streamlining of neutrons through the beam ports and other penetrations in the large chamber.

The deep penetration of neutrons through the bulk shield is a relatively common deeppenetration problem. In principle, the bulk shield can be designed to be thick enough to reduce the neutron leakage to any specified level.

Benchmark experiments to verify codes and data can help reduce uncertainty, the safety factor for design, and hence the cost of the bulk shield.

- The streaming of neutrons through the beam ports and other penetrations in the target chamber is quite unique to IFE reactors, due to the large number of penetrations and to their radial orientation (with their axis crossing the target). The activation and radiation damage to components which are in the "line of sight" of the IFE target is relatively straightforward to address, since the neutron flux is well defined. It is more complicated to reliably predict the neutron field in out-of-chamber, out-of-line-sight components. Penetrations contribute to out-of-chamber component activation via scattering in the optical and structural components located in the beamline and scattering in the chamber wall and shield in the vicinity of the penetrations. The specific geometry and materials to be used in the shield (and blanket) regions adjacent to the 
penetrations will have a significant effect on the neutron leakage probability. Due to the complicated nature of these neutron interaction pathways, benchmark experiments aimed at verifying our ability to accurately account for the effect of different penetration shield materials and geometries on the leakage enhancement pathways will be essential.

For ion beam driven IFE reactors, the leakage neutrons can induce radiation damage to the super-conducting magnets, which surround the beam ports (the purpose of which is to focus or to bend the beam). In such reactors, it is essential to be able to accurately characterize the neutron field in the vicinity of magnets.

In indirect-drive IFE targets such as heavy ion targets, it may be possible to significantly reduce the fraction of fusion neutrons which leak through the beam ports by using in-vessel placed liquid jets or solid pencil-shaped objects attached to the target. This "neutron beam stopper" will use two unique features of indirect-drive targets: 1) their small volume of origin $(<<1 \mathrm{~mm})$ at the center of the capsule (see Fig. 3.2-1), and 2) the fact that the beam aim point is separated from the small dimension neutron source point by about $10 \mathrm{~mm}$ (so that the neutrons streaming towards the beam port follow a slightly different path than the ion beam path). The NIF could provide the first opportunity to experimentally verify the feasibility of this concept by using one of the ion targets shown in Fig. 3.2-1, with lasers aimed at the two converters, from two-off axis sets of beams.

The NIF will provide intense enough neutron pulses and representative geometries to perform benchmark experiments which are expected to resolve most of the shielding issues of importance for the design of IFE reactors which will follow the NIF. Specifically, we are proposing to carry out the following shielding related experiments with the NIF: 1) activation and dose measurements throughout the out-of-chamber volume, 2) addition of a dummy beam tube assembly combined with a provision to install and replace different penetration shield modules to study the effect of different penetration shield materials and geometries on the out-of-chamber source of neutrons, 3) bulk shield experiments, and 4) use of liquid jets or equivalent pencilshaped solids to shield against the streaming of neutrons through the beam ports.

\subsubsection{Thermomechanical Structural Response}

NIF can provide important information relevant to some of the thermomechanical structural response of the vacuum vessel and the in-vessel components. For the vacuum vessel, information can be obtained to assess the effect of multiple penetrations on the integral response of the vessel to a 2-20-MJ point source of energy release. Examples of information to be obtained include in-vessel strain rate, stress concentration, vibrational modes, displacements, and energy damping rate. Such information can be used to partially benchmark design codes for IFE.

Information can also be obtained on the effects of a multi-MJ point source of energy release on the survival of in-vessel components. Examples of information to be obtained include temperature gradient, wave propagation, and effect of support mode. This information can help determine damage threshold survival rate for invessel components.

\subsubsection{Reliability and Remote Maintenance}

Remote maintenance capabilities should be designed with NIF, as they were for Joint European Torus (JET). They should be practiced during routine operation to develop efficient procedures, and to uncover flaws of hardware, software, and procedures. Component failure rate data should be collected on NIF components. An extensive component failure rate database will be needed as a prerequisite for doing probability/risk assessments essential for estimating accident frequencies.

\subsubsection{Safety}

As we consider safety issues for an eventual IFE power source, the main concern is protection of the workers, public, and environment, including the consequences of accidents, exposures to radioactivity or toxic materials from routine operations and waste products. Specific 
issues include tritium and activation product inventories and gamma shielding to reduce doses to personnel. The standard industrial safety issues of asphyxiation, electric shock, high pressures, burns, and toxic chemicals must also be taken into account. Keeping in mind the future decommissioning and decontamination of an IFE reactor, we should try to minimize the quantities of radioactive waste and hazardous wastes that it generates, and try to recycle target materials (such as tritium and heavy metals) as much as possible.

NIF operation can provide useful data on routine tritium emissions, on tritium inventories in reactor components, and on the effects of neutron steaming on personnel doses. We can look for the production of mixed hazardous/ radioactive waste during NIF operation, devise strategies that minimize waste. We need to verify predictions of activated materials produced from target materials, structural materials, or other components unique to IFE. For example, we can devise strategies for dealing with potential boron toxicity.

Coupons can be placed in NIF to study the effects of tritium implantation, trapping, and release, as well as surface modification, radiation damage, and segregation of activation products.

Experience has shown that a key element of overall facility safety is the existence of adequate remote instrumentation that operators would need in responding to emergencies. Such safetyrelated diagnostics can be developed during NIF design and operation.

Some additional technology development projects that are not required as experiments for NIF but that should be done in parallel with NIF to prepare for the DEMO that will follow. These include

- Demonstration of IFE coolant purification, buffering, and recycling of heavy metal target materials

- Demonstration of tritium removal from IFE coolants

- Development of techniques for tritium removal from potential IFE reactor structural materials.

The NIF is not unique for IFE safety and environment studies, but safety/environment analyses and design features are essential for the success of NIF.

\section{NIF Environmental and Safety Considerations}

Our analyses, presented in documents available to the general public, ${ }^{11-13}$ have determined that the NIF will present negligible environmental or safety risk to the general public or the NIF work force.

\section{Radiological Aspects}

Natural background radiation from our environment (naturally occurring elements, cosmic radiation, etc.) is approximately $0.3 \mathrm{rem}$ per year. This does not include exposure to other sources of radiation such as airplane flights and medical diagnoses.

- The routine annual dose due to NIF at its site boundary is expected to be $0.00013 \mathrm{rem}$, or $0.13 \%$ of the DOE/EPA guideline, and is $350 \times$ less than the radiation dose from a $1-G W_{e}$ coal-fired power plant.

- The average annual additional dose to a NIF worker will be about 0.01 rem, while the maximum for any worker will be $<0.5 \mathrm{rem}$. This is $<10 \%$ of what is allowed under DOE guidance. The average annual dose received by flight attendants is 0.5 rem, by comparison.

- The maximum tritium inventory for the NIF facility will be $300 \mathrm{Ci}(0.03 \mathrm{~g})$. (Naturally occurring tritium in the atmosphere, produced by cosmic ray interactions, totals $700 \mathrm{~g}$ ). The NIF inventory is $<3 \%$ of the routine inventory of the National Tritium Labeling Facility (used for tagging biomedical samples) in Berkeley, CA. A single NIF target will contain $<2 \mathrm{Ci}$ of tritium, $1 / 5$ the inventory of a typical theater exit sign. More than one million such signs are sold each year.

- There are no significant radioactive or hazardous effluent levels for NIF. For example, the projected maximum emission of tritium is $<10 \mathrm{Ci} / \mathrm{y}$, or the equivalent of the tritium contained in a 
single exit sign. The annual dose from all NIF effluents is $600 \times$ less than that from a single cross country airline flight.

- The worst-case accident considered in our safety assessment assumes the release of all of the tritium $(300 \mathrm{Ci})$ in its worst biohazardous form, tritiated water. The effect of a 20-MJ yield experiment (the maximum yield) is also included. This postulated (yet highly unlikely) accident would result in a dose of 0.056 rem at the NIF site boundary, or $0.2 \%$ of the DOE siting guidelines.

\section{Waste Generation}

For conservatism, we are reporting quantities larger than expected for the waste streams for NIF. Our assessment has yet to fully benefit from the application of waste minimization techniques, such as $\mathrm{CO}_{2}$ cleaning, which will be an important continuing design activity after Key Decision 1. All of the NIF projected waste is included in the approved 1992 EIS/EIR ${ }^{14}$ for the LLNL site.

- The annual hazardous waste stream is about $3200 \mathrm{~kg}$. The majority of this stream, about $2300 \mathrm{~kg}$, is comprised of 20 boxes of paper-soaked capacitor oil, similar in composition, but less in quantity, than that generated over the same time period by an automobile oilchanging facility. Certified contractors will routinely dispose of this NIF waste.

- The annual mixed waste stream, $270 \mathrm{~kg}$ of solid and $3.8 \mathrm{~m}^{3}$ of liquid, a small fraction of the quantity generated today at LLNL, will be significantly reduced (and the liquid mixed waste perhaps even eliminated) by the use of $\mathrm{CO}_{2}$ cleaning.

- The annual solid low-level radioactive waste stream, $900 \mathrm{~kg}$, also a small fraction of the quantity currently generated at LLNL, will be less than $1 / 6$ of that produced by a major university.

- The liquid low-level radioactive waste stream will be disposed of in accordance with applicable guidelines.

\section{Filling Targets with Tritium}

ICF targets have been, are, and will be used in many ICF facilities throughout the country, such as Nova, PBFA-II, Omega Upgrade (future), and NIF (future). The research and fabrication of targets, including some which require the filling of capsules with deuterium and tritium, is a stand-alone activity of the national ICF Program providing support for all ICF facilities. Target activities are conducted at several sites [e.g., General Atomics (La Jolla, CA), W. J. Schafer Associates (Livermore, CA), University of Rochester (NY), LLNL, and LANL]. Since this national capability already exists, NIF will not include a dedicated target facility, but will receive targets from these other sites. Less than $5 \mathrm{~g}$ of tritium will be needed in a facility that provides fusion capsules for NIF. These target facilities have their own safety and environmental documentation, and systems engineered to protect workers, the public, and the environment by safely confining their tritium. NIF targets will be transported in prescribed Department of Transportation certified containers, and in accordance to regulations (49 CFR 173).

\section{Conclusion}

Based on its review of the Preliminary Hazards Analysis for the National Ignition Facility, ${ }^{2} \mathrm{DOE}$ Oakland concurred with the categorization of the NIF as a low-hazard, non-nuclear facility, meaning that the operation of the NIF will have minor onsite and negligible off-site consequences. This hazards categorization will be reviewed after each subsequent safety analysis report.

\subsection{Target Systems}

\subsubsection{Introduction}

Most IFE target requirements will be developed in an IFE target development program separate from the NIF, but the NIF can provide some important performance tests of candidate 
IFE targets. IFE target systems include two areas:

1) target fabrication (target materials and configuration selection, capsule production, hohlraum production, target assembly, characterization, fill, and layering); and 2) target transport (transport from the target factory to the target chamber, and target insertion, tracking, and protection inside the target chamber). With regards to the above two areas, we discuss the issues that must be resolved to successfully develop an inertial fusion energy power plant, and the experiments that could be done in the NIF to help resolve these issues.

\subsubsection{Target Fabrication}

Fabrication Issues with IFE Targets. The targets that fuel an inertial fusion power plant based upon the indirect-drive approach will be similar to the ignition and high-gain targets developed for the NIF. The IFE fuel capsules, however, will be two-to-three times larger in diameter since capsule size scales according to the cube root of the absorbed energy. For a laser driver, hohlraums will scale similarly. Ion drivers will require substantially different hohlraums and $x$-ray converters. As with NIF high-gain targets, IFE targets will be cryogenic. The major issues associated with developing these targets, broadly stated, are: 1) assuring the target component quality specifications as sizes increase and fabrication techniques change; 2 ) assuring a fast enough fuel-fill rate to be able to maintain the plant tritium inventory at acceptable levels; and 3) developing fabrication and inspection techniques that produce high-quality, economically viable targets. A fourth issue, dealt with in the following section on target transport, is providing targets that can withstand the * accelerations attending chamber injection and the chamber environment once injected.

Table 3.5-1 lists more fine-grained issues that must ultimately be addressed to produce and use IFE targets, together with an assessment of the importance of the NIF in developing solutions. In summary, most IFE target requirements can be developed "off line." Those that require full scaling to IFE sizes, such as capsule surface morphology, cannot be completely tested to ignition on the NIF. Those that do not require such scaling, such as changes in capsule material or target assembly techniques, should be tested on the NIF. The NIF would be a unique testbed, since the relevant test of success is ignition.

Currently, each target for ICF experiments costs thousands of dollars to fabricate. For a . commercially viable IFE power plant, however, the cost per target must be around thirty cents. Immense cost reductions will accompany an increase in production rate from roughly $1000 / \mathrm{yr}$ at present to $10^{9}-10^{10} / \mathrm{yr}$ needed for an IFE-based fusion economy. This reduction will require using cheaper mass production techniques and perhaps some changes in materials. Savings may also be realized from a relaxation of target

Table 3.5-1. Target fabrication issues.

\begin{tabular}{lcc}
\hline \multicolumn{1}{c}{ Issue } & $\begin{array}{c}\text { NIF } \\
\text { usefulness }\end{array}$ & $\begin{array}{c}\text { NIF } \\
\text { uniqueness }\end{array}$ \\
\hline \hline $\begin{array}{l}\text { Low-cost mass-production techniques for capsules and their } \\
\text { effect on quality, materials choice, and gain }\end{array}$ & 2 & 3 \\
Low-cost mass production of laser driver hohlraums & & 3 \\
The effect of cryogenic layer quality on gain & 2 & 3 \\
Automated cryogenic assembly techniques & 2 & 3 \\
Fast fill techniques for.low tritium inventory & 3 & 3 \\
High-throughput quality inspection techniques & 2 & 3 \\
* Usefulness: $3=$ Complete resolution, $2=$ Partial resolution, 1 = Useful information, $0=$ No use. \\
** Uniqueness: $3=$ NIF unique and required, $2=$ NIF not unique but could be used, 1= Issue addressed better or \\
cheaper in new facility, $0=$ Issues addressed better or cheaper in existing facility.
\end{tabular}


parameters or development of new target configurations that may allow easier fabrication. The first three rows of Table 3.5-1 contain issues of low-cost production. They cannot be completely resolved for IFE targets due to limitations in the NIF output energy.

The fourth and fifth items in Table 3.5-1 address minimizing the tritium inventory. This requires developing a fast fill technique (e.g., drill, fill and seal) or a capsule material with very high permeability. Capsules would have to be filled prior to assembling into hohlraums to minimize the volume of the batch filling apparatus. Thus, since the capsule will very likely have to be kept at cryogenic temperatures to avoid rupture, a technique would have to be developed for cryogenic assembly of the capsule into the hohlraum.

The last item in Table 3.5-1 would investigate the range of acceptable target component qualities. Broadening this range would not only help reduce fabrication costs, but would considerably ease the inspection requirements, which may be essential for high throughput.

In Table 3.5-1, we have not included issues specific to heavy ion driven targets. However, NIF experiments relevant to these targets are proposed in the section on Target Physics, and may require additions to the table. In any case, the generic issues of low-cost, low tritium inventory, and high target quality apply to these targets as well. Finally, as mentioned above and explained more fully later, high rep-rate injectable targets may require additional robustness, whose effects on ignition performance would have to be tested on the NIF. The issues surrounding injection of targets are summarized in Section 3.5.3.

Proposed Target Fabrication-Related Experiments. Targets for NIF ignition and highgain experiments will be fabricated with the best available technology. Clearly IFE targets cannot receive the fabrication attention that ICF targets get. But there will also be limits on the compromises that can be made in the fabrication of IFE targets without decreasing their performance to the point where their gain drops unacceptably (or they won't ignite). An experimental series exploring the effects on the target gain of the fabrication modifications dictated by the economics of IFE would be very useful to IFE.

We propose to test the effect of cheaper hohlraum fabrication techniques (e.g., stamping, drawing, and spinning) on target performance. Alternate materials that would aid in cost reduction would be tested. Similarly, the proposal would probe the performance of capsules selected that would aid in cost reduction by cheaper, automated characterization techniques for wall thickness, uniformity and fill pressure. These and other deliberate (and wellcharacterized) defects could be included such as imperfections in material boundaries and point defects in capsules. These effects could be tested separately and in combination, by selectively ircluding components made via proven methods. All these targets would be characterized extensively in the usual ways to provide quantitative measures of the fabrication compromises. The goal would be to define the performance costs of mass production techniques suitable for IFE, where target costs have a significant effect on plant economics. An appropriate number of shots for each variation (several hundred over 4-6 years) would be compared to the data set for high-gain targets to provide statistically significant limits on IFE fabrication techniques.

\subsubsection{Target Transport}

Target Transport Issues. Under the category of target transport, we include both transport from the target fabrication facility to the target injector, and the injection of the target to the point of ignition. Completed targets will be stored in a cryogenic storage system prior to transport to the injector; the targets must be kept at constant temperature throughout the entire transport and injection process. Even after the target leaves the injector and enters the chamber environment, the allowed temperature rise of the cryogenic fuel is very low, estimated to be less than $0.2 \mathrm{~K}$. Targets must also survive the acceleration process. The target transport issues for IFE are listed in Table 3.5-2. 
Table 3.5-2. Target transport issues.

\begin{tabular}{lcc}
\hline \multicolumn{1}{c}{ Issue } & $\begin{array}{c}\text { NIF } \\
\text { usefulness }\end{array}$ & $\begin{array}{c}\text { NIF } \\
\text { uniqueness** }\end{array}$ \\
\hline \hline Injection techniques for-high-rep rate cryogenic operation & 0 & 1 \\
Time and space accuracy and sensing & 0 & 1 \\
Integration & 2 & $1-2$ \\
Target survival under acceleration & 2 & 3 \\
Thermal protection and temperature control & 2 & 2 \\
Chamber environment effects on trajectory & $1-2$ & 3 \\
Demonstration of high-rep rate operation & 2 & 3 \\
\hline
\end{tabular}

- Usefulness: 3 = Complete resolution, $2=$ Partial resolution, $1=$ Useful information, $0=$ No use.

** Uniqueness: 3 = NIF unique and required, $2=\mathrm{NIF}$ not unique but could be used, 1 = Issue addressed better or cheaper in new facility, $0=$ Issues addressed better or cheaper in existing facility.

To proceed with development of inertial fusion for energy, we must design and develop a target handling system to transport targets from the fabrication facility to the injector on the reaction chamber. This must operate at cryogenic temperature, operate at the required rep rate $(\sim 1-10 \mathrm{~Hz})$, and operate without damage to the target or sabot. In addition, we must design and develop a high-pulse rate injector that is similarly capable of cryogenic operation at a high rep-rate, that will operate without mechanical or thermal damage to the target, and may involve use of a sabot. This injector must exhibit high reliability and highly repeatable accuracy. To be able to fire the driver at the right time, we must design and develop a target tracking system that will detect the target in transit in chamber; the target tracking system must be capable of providing adequate position and timing information to the driver. These tracking systems must be protected from the radiation environment of the chamber. The effect of the postshot environment on next target is also of concern. The target must be protected from the effect of thermal radiation from chamber walls and from convective heat load from residual gases; the target must be protected from the radiation pulse of the prior shot while waiting for injection. In addition, the effect of chamber vapor on the target trajectory must be accounted for.

Finished targets must be handled in a way that does not degrade the quality with which they were manufactured. The entire handling and transport to the target injector must be designed to operate at the required pulse rate and at cryogenic temperatures. This will involve conveyors and robotic handling equipment, which need to be developed and demonstrated.

Several options for injectors have been proposed including gas guns and electromagnetic launchers. The key issues are to design and demonstrate a highly reliable system capable of operating at high rep-rate with cryogenic targets. The targets must be protected from damage during the acceleration process and during transit through the chamber. Protective sabots, which detach from the target prior to ignition, have been proposed for protection during injection. These must be demonstrated.

Conceptual solutions to target tracking and beam pointing have been developed. Most rely on tracking the target before it enters the chamber, so the tracking systems can be protected from the radiation environment. Tracking systems that can provide position and timing information that will precisely illuminate the target must be developed.

IFE chambers use either liquids or gases to protect the first wall from damage by short ranged target emissions. The vaporization and condensation of liquids or blast waves in gas protected chambers create a dynamic environment that results in heat loads to the target (radiative and convective); this condition may affect the trajectory of the next target in a nonuniform manner. Radiation from the hot 
chamber walls could lead to excessive heating of the target prior to ignition. The thermal capacity of the target may be sufficient to keep the fuel layer from overheating (especially for hohlraum targets), or a sacrificial frozen gas layer might be added to the outer surface of the target.

Proposed Target Transport-Related Experiments. While most of the required development for target transport must be developed off line and is not needed for NIF, actual integration and demonstration will require a reaction chamber environment. Further, some aspects of target transport systems may be useful to NIF.

Target Alignment and Positioning. We propose to measure the performance penalty for target positioning errors. Targets for IFE applications would be placed into the target chamber with intentional alignment and positioning errors. Targets would be illuminated in other than the optimal geometry. A series comparing the performance of deliberately misaligned targets would delineate the gain penalties of targets that were displaced from chamber center. High-gain targets would be positively positioned above (or below) the ideal focal position; in the mid-plane but off-axis from the ideal illumination location, a combination of the two; and displaced angle from a axial location. The performance of these shots would be compared to the database of targets illuminated in the ideal location with the goal of determining the required beam pointing and target aiming for IFE target injection systems. A total of about 50 shots would be required. These experiments would provide performance specifications for the target injection experiments described below. Combined injection/ positioning experiments may be possible.

Target Injection. The ability to ignite targets on the fly is essential for inertial energy power plants. Once components of inertial fusion energy target injection have been developed off line, they could be demonstrated in an integral manner on NIF.

Three experiments are proposed for injecting targets on NIF which should first be developed and demonstrated off line. The first involves dropping a cryogenic target into the NIF chamber. The target falls through a laser timing gate at a point between the release point and the center of the chamber. This detector provides timing information for initiating the laser pulse. The target would probably be released from within the chamber to minimize target heating and more easily control target position.

The second proposed experiment is more closely related to expected IFE target injection procedures. A gas gun is used to accelerate targets into the fusion chamber. Photodiodes are used to track and time target arrival. If sufficient target position repeatability is not achieved by this method, it may be possible to electrostatically steer the targets onto the correct trajectory.

The third experiment reduces target heating. A thin frozen layer of neon (or other suitable material) is adhered to the hohlraum. During injection, this material will ablate off the surface reducing target heating. We will measure the effect of possible nonuniform ablation on target trajectory. An alternate method of minimizing the heating of a falling target is to drop the target through a cryogenic tube during the initial slow moving part of the fall.

Remote target injection methods may also be beneficial to NIF. In the baseline design, cryogenic cooling tubes lead to the target. The tubes will be destroyed; they will produce shrapnel debris with each shot. Replacement cost and potential shrapnel damage could be avoided by remotely injecting targets and igniting them on the fly. This would be accomplished at the expense of reduced target position certainty.

Target Chamber Environment. NIF offers a chance to study postshot environment under conditions that could be extrapolated to an IFE power plant. The influence of the chamber environment on the injection of subsequent targets and beam transport is critical to successful operation of an IFE power plant.

We propose an experiment to look at the repeatability and accuracy of various target injection schemes. The $5-\mathrm{m}$ NIF vacuum chamber is roughly the same size as estimates of chambers needed for operational IFE power plants. Initial studies can be done concurrently on an injection shot immediately after an ignition shot. The idea is not to fire the laser at the injected target, but to determine the effects of the postshot environment 
on the injected target (specifically trajectory and target temperature). The test target would be injected 0.05-0.2 $\mathrm{s}$ after the shot. This could present the injected target with conditions that would be present in an IFE power plant operating at a 5 to $\sim 10 \mathrm{~Hz}$ rep-rate. For trajectory experiments, the postshot injected target need not be cryogenic, but should have the same size and mass as a real target. Injection schemes suitable for both direct and indirect drive should be considered. Instrumentation would include diagnostics to measure gas pressure, species, clearance time (due to vacuum pumping); chamber temperature and profile; target position, orientation and velocity; and, if possible, temperature and inner surface quality of a cryogenic target. This last measurement should first be done off line or in a static experiment after the chamber conditions are determined and simulated.
If fluence conditions equivalent to that of an IFE power plant are desired, a dedicated series of shots would be made by inserting a 1-m-radius chamber (with holes for the laser beams) inside the NIF chamber $\left.[20 \mathrm{M}] \times(5 / 1)^{2}=500 \mathrm{M}\right]$ simulation]. This condition is illustrated in * Fig. 3.5-1. Nonvacuum conditions (e.g., 1 torr of neon gas consistent with the ability to fire the NIF lasers) could also be studied.

Before experiments of any kind can be done on NIF, R\&D will be needed to develop ignition targets. To develop targets for IFE and costeffective fabrication techniques for these energyrelevant targets, and to develop target transport systems for IFE, will require further effort. Some of this effort will be needed before IFE target systems experiments can be fielded on NIF. See Table 3.5-3.

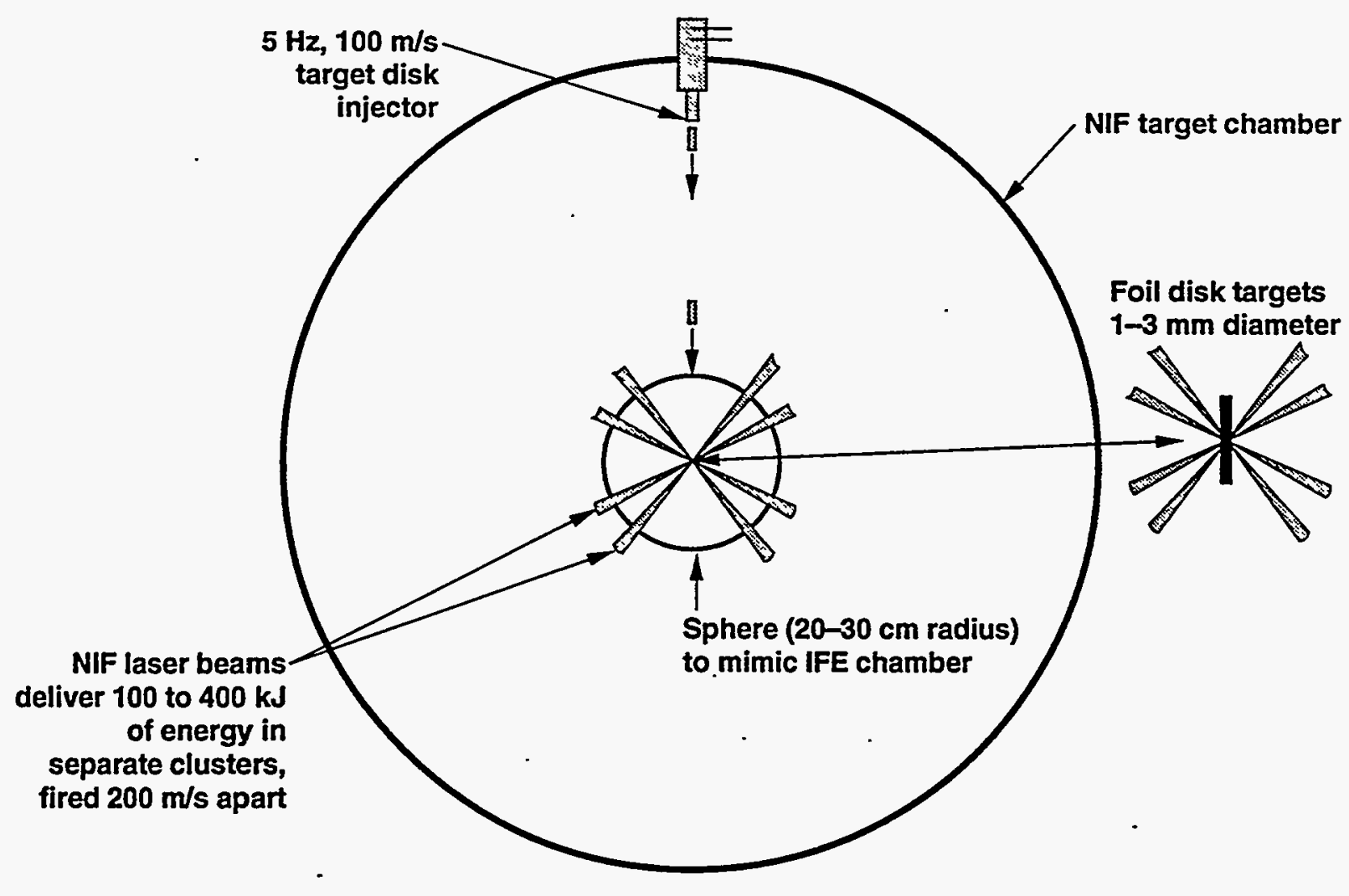

70-37-0195-0002pb01

Figure 3.5-1. A 1-m radius inner chamber is used to simulate the fluence of a full-scale IFE chamber. 
Table 3.5-3. Target systems $R \& D$ needs.

\begin{tabular}{|c|c|c|c|c|}
\hline $\begin{array}{c}-\because \\
\text { Development item }\end{array}$ & $\begin{array}{l}\text { Needed for } \\
\text { NIF }\end{array}$ & $\begin{array}{l}\text { Needed for } \\
\text { IFE } \\
\text { experiments } \\
\text { in NIF }\end{array}$ & $\begin{array}{l}\text { Needed for } \\
\text { IFE }\end{array}$ & $\begin{array}{l}\text { Current } \\
\text { R\&D } \\
\text { activity* }\end{array}$ \\
\hline \multicolumn{5}{|l|}{ TARGET FABRICATION } \\
\hline \multicolumn{5}{|l|}{ Targets for ignition } \\
\hline Ignition target design & $x$ & & & A \\
\hline $1-3 \mathrm{~mm}$ capsules & $x$ & $\mathrm{X}$ & $x$ & B \\
\hline High-pressure DT fill and condense & $x$ & $x$ & $x$ & $\mathrm{C}$ \\
\hline Cryogenic layering & $x$ & $x$ & $x$ & $\mathrm{D}$ \\
\hline Cryogenic characterization & $x$ & $x$ & $x$ & $\mathrm{D}$ \\
\hline Cryogenic assembly & $x$ & $x$ & $x$ & - \\
\hline \multicolumn{5}{|l|}{ Targets for IFE } \\
\hline IFE target design & & $x$ & $x$ & - \\
\hline IFE target fabrication & & $x$ & $x$ & - \\
\hline Cost-effective fabrication & & & $x$ & - \\
\hline \multicolumn{5}{|l|}{ TARGET TRANSPORT } \\
\hline \multicolumn{5}{|l|}{ Transport systems } \\
\hline Transport to reaction chamber & $x$ & $x$ & $x$ & C \\
\hline High-throughput transport & & & $x$ & - \\
\hline \multicolumn{5}{|l|}{ Injection and tracking } \\
\hline Stationary mounting system & $x$ & $x$ & & $\mathrm{C}$ \\
\hline Free-fall injection & & $x$ & & - \\
\hline High-speed injection & & $x$ & $x$ & - \\
\hline $\begin{array}{l}\text { High-rep rate, radiation hardened } \\
\text { injection }\end{array}$ & & & $x$ & - \\
\hline Target tracking & & $x$ & $x$ & - \\
\hline Hardened target tracking & & & $\mathrm{x}$ & - \\
\hline $\begin{array}{l}\text { A: NIF Design Activity } \\
\text { B: Target Fabrication Development Activity } \\
\text { C: OMEGA Upgrade Design } \\
\text { D: National Cryogenic Target R\&D Program }\end{array}$ & & & & \\
\hline
\end{tabular}

\subsubsection{Target Systems Research and Development Needs}

Target Fabrication. Designs exist for ignition targets. Some development will be needed to fabricate these targets; additional designs will be needed in the course of the NIF experimental program. Preliminary designs exist for IFE targets, but more detailed designs must be made and fabrication techniques must be developed before such designs could be tested in NIF.

Targets for Ignition. The present designs for NIF ignition targets are very similar to current targets shot on NOVA, just larger and cryogenic. Some development, however, will be required. Target quality capsules about $1-3 \mathrm{~mm}$ in diameter will be needed, and are not currently available. A method is needed to fill these 
capsules with DT to about 1000 atm pressure, and then cool them to cryogenic temperatures to condense the DT. The DT layer inside the capsules must meet stringent specifications for uniformity ( $>99 \%$ ) and sürface roughness $(<5000 \AA)$. R\&D is underway as part of the National Cryogenic Target R\&D Program to develop these capsules. Techniques for assembly of cryogenic capsules and hohlraums, or a method to fill capsules while they are inside ignition target hohlraums, are needed. These development needs must be filled for the NIF itself, immaterial of IFE experiments. Some of the capsule and cryogenic capsule fill needs are being addressed for cryogenic targets for the OMEGA Upgrade experiments at the University of Rochester. This work will directly benefit NIF and ultimately IFE. Work on hohlraums and cryogenic assembly, however, is not yet being done, and will be needed for NIF. This effort will then directly benefit IFE.

Targets for IFE. Targets developed for IFE must be inexpensive, robust for handling, transport and injection, and compatible with the chamber environment of an IFE power plant. Target materials, fabricat ion techniques and fill methods for IFE will surely be different than those currently envisioned for the NIF ignition targets. Prototypes of such targets must be developed and fabricated for testing on NIF.

We recommend that studies be done to design IFE targets. These studies would be analytic and would be relatively inexpensive. Designs relevant for testing on NIF would then be selected, and prototype targets fabricated for NIF experiments. These prototypes would exhibit the features of IFE targets that are different from those needed for ignition alone, and would examine the effect on yield of features required for IFE. Actual development of mass production capability for such targets and demonstration of target fabrication economics will require further effort and equipment, but is not needed for the experiments on NIF.

Target Transport. To be able to do ignition experiments on NIF will require cryogenic target transport systems to mount targets in the reaction chamber. Large-scale, high-throughput, highly reliable target transport, injection and tracking technologies will be needed for IFE. The experiments proposed for NIF will not require large numbers of targets, nor the automation or reliability needed for IFE. Nevertheless, some development will be needed on injection and tracking techniques to field the experiments on NIF. This development will be directly relevant to IFE, and in fact, NIF will play an important role in this development.

Transport Systems. To get cryogenic ignition or prototype IFE targets from the fill station to the center of the target chamber will require cryogenic target transport systems. These must be part of the NIF experiments, and will probably not be in any way prototypic of transport systems for IFE. Development of such systems for NIF will be able to build on the development of the cryogenic target transport system for OMEGA Upgrade.

Injection and Tracking. Ignition experiments on NIF will utilize stationary mounted targets; no injection or tracking is needed. IFE will require sophisticated target injection and tracking systems to inject targets into the hostile environment of the reaction chamber at the rate of $1-10 \mathrm{~Hz}$. The IFE target system experiments proposed for NIF will require partial development of some of these systems. A free-fall target injector will not be adequate for IFE, but it may work for NIF and is a first step toward the high-speed injection system that will be needed for IFE. Development of the injection mechanisms for free-fall and high-speed injection, and development of the tracking and firing systems to use these injectors will be needed for the proposed experiments to be fielded on NIF. The high rep-rate, high reliability, and radiation hardening that will be required for IFE will not be required for experiments in NIF.

We recommend that studies be done to design target injection and tracking systems for IFE application. Based on these studies, appropriate tests on NIF can be planned, and the development needed to field this hardware can be carried out. By making it possible to move all hardware farther from the target ignition point, target injection systems for NIF may help facilitate other experiments and reduce the 
effect of blow-off from the target stalk on

NIF diagnostics.

Summary of Development Needs. Target fabrication and injection systems for IFE will require development. Some of the target fabrication issues must be faced in order to field ignition targets on NIF; this effort will require continuation and expansion of current target development activities. The testing proposed in NIF of IFE-relevant targets will require that those targets first be developed. Relatively little has been done in that area to date. We recommend a program of target design and fabrication R\&D to have prototypic IFE targets ready for testing on NIF. This testing will come after the NIF primary mission of ignition has been achieved.

Cryogenic ignition experiments on NIF will require cryogenic target transport systems.

Development of these systems will benefit greatly from work on similar systems being developed for OMEGA Upgrade. IFE will require sophisticated target injection and tracking systems that have yet to be seriously studied. IFE target experiments on NIF will require a portion of the development needed on these systems to be done. We recommend that IFE tracking and pointing studies be done to define the systems that could be tested on NIF, followed by development of the required hardware for these experiments. These experiments would logically follow the static tests of IFE prototype targets described above.

\subsubsection{Summary for Target Systems}

The Target Systems session of the Workshop on NIF Experiments for IFE reviewed the target systems design results from several recent IFE power plant design studies; the workshop developed a list of the critical issues that needs to be resolved before these designs could be successfully built and operated with confidence. These issues can be broadly categorized into two areas: target fabrication issues and target transport issues. We reviewed a number of proposed NIF experiments and found that the " NIF can play an important role in developing target systems, and in resolving critical target system issues for IFE.

For the most part, the target fabrication issues must be resolved by development work done in the laboratory, not in NIF; many must be resolved before the NIF can be successful. However, the investigation of how target yield varies with target design changes (needed to develop economical, mass-produced targets for IFE) will have to be done in actual implosion experiments; the NIF is needed for such experiments. While these NIF experiments can be done with minimal impact on the design of NIF, several hundred high-yield shots could be needed, which could impact the NIF operating schedule and planned lifetime.

Target transport issues related to target handling, insertion and tracking must also be acldressed by development work in the laboratory. However, integration of these components into a complete system, and demonstration of this system could be done most convincingly on the NIF. Use of a target injection system may, in fact, be useful to NIF in that the effect of target insertion system hardware (blowoff and debris) on NIF experiments could be reduced. The effect of inertial fusion reaction chamber conditions on the survival and performance of targets must be determined under actual reaction chamber conditions; experiments in NIF could help in this effort. The target transport issues experiments will require insertion system hardware and an inner chamber to simulate IFE postshot conditions be added to NIF, however, this equipment will have little impact on the basic NIF design. The total number of shots needed for these experiments should be less than 100 .

In short, much of the development work needed for IFE target systems must be done in the laboratory, not on an implosion experiment in NIF. However, experiments done in the NIF could play a valuable role in developing targets for IFE, and in verifying performance of IFE target injection systems. These experiments should have minor impact on the basic design of NIF, but could require several hundred dedicated, high-yield shots. 


\subsection{Use of the NIF to Test Heavy ION Target Physics}

\subsection{Introduction}

The current DOE strategy in establishing the feasibility of IFE requires two primary demonstrations: 1) Defense Programs (ICF) will demonstrate inertial fusion ignition and energy gain through the NIF, based on a glass laser; 2) Energy Research (Office of Fusion Energy) will demonstrate the feasibility of an IFE driver with heavy ion accelerators (recommended by the 1990 FPAC review) through the completion of the ILSE program. This strategy raises the question of the relevance of NIF laser-driven target physics to heavy ion targets designed for heavy ion drivers. Section 3.2 indicated the generic IFE target physics issues that NIF target experiments could address for both ion (light and heavy ions) and laser drivers, and for both direct and indirect drive. The NIF laser and its associated technology development also has a very significant relevance for a solid state laser IFE driver option. A diodepumped solid-state laser (DPSSP) could be a viable IFE driver option if diode costs can be reduced further and if target gains can be increased (see Section 5.5). However, this section will be limited to discussing how NIF can be used to validate heavy ion targets, since heavy ion accelerators are the primary driver development now being supported by Energy Research for IFE. In particular, we will discuss how the achievement of ignition in the NIF (the ICF ignition campaign for defense applications) will demonstrate understanding and control of fuel capsule implosion physics to a degree even greater than required for adequate performance of a heavy ion target in an IFE power plant, and furthermore, how experimental techniques recently developed on NOVA can be exploited in specific NIF targets designed to better simulate the hohlraum plasma and $x$-ray transport physics in a NIF-like hohlraum geometry.

\subsection{A Heavy Ion Target Point Design for IFE}

To understand the relevance of NIF ignition tests to heavy ion IFE targets, we first briefly review the heavy ion point design of Ho et al. ${ }^{1}$ Figure 4-1 is the baseline heavy ion target configuration, which is axisymmetric and illuminated by two clusters of heavy ion beams (HIB) coming from the two ends. The heavy.ion beams penetrate the thin high- $Z$ radiation cases surrounding the $\mathrm{Pb}$-doped beryllium converters of radius $r_{c}$, which heat up and convert the heavy ions' kinetic energy into soft $x$-ray radiation. The soft $x$-ray radiation diffuses out of the channels of radius $r_{0}=1.5 \mathrm{~mm}$ and into the main hohlraum cavity of $7-\mathrm{mm}$ radius, also made of lead. The high soft x-ray reflectivity (albedo) of the lead

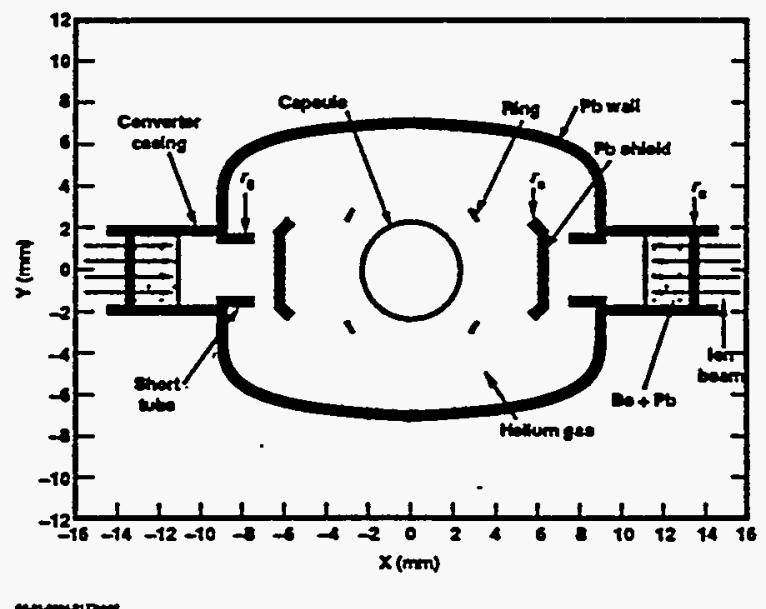

Figure 4-1. Baseline HIB target. 
walls allows the radiation to spread around the hohlraum cavity with a significant fraction of the $x$ rays uniformly absorbed by the DT fuel capsule in the center of the hohlraum. The soft $x$-ray ablation of the outer layer of the capsule creates a high ablation plasma pressure, which implodes the inner DT fuel layer of the capsule to densities and temperatures sufficient for fusion ignition and gain.

A pair of lead radiation shields on the axis of symmetry, plus an additional pair of annular lead rings near $45^{\circ}$ reduce the time-averaged $\mathrm{P}_{2}$ and $\mathrm{P}_{4}$ asymmetries of the soft $x$-ray flux on the capsule $\left(P_{n}\right.$ refers to Legendre polynomials of order $n$ ). The capsule and lead shields are to be supported by thin plastic membranes or other transparent support structures (not shown) of sufficient strength to survive acceleration of the target when injected. ${ }^{2}$ For simplicity, the effects of the plastic membranes on hohlraum soft-x-ray transport and symmetry are ignored in this work, but will need to be incorporated in future HIF target designs.

Figure 4-2 is the fuel capsule structure. The beryllium ablator absorbs $1 \mathrm{MJ}$ of soft $\mathrm{x}$ rays to drive the capsule implosion producing a fusion yield of $430 \mathrm{MJ}$. Figure 4-3 shows the hohlraum radiation temperature profile required for the implosion, which peaks at $260 \mathrm{eV}$. Although beryllium is assumed for both the radiation converters and capsule ablators in these

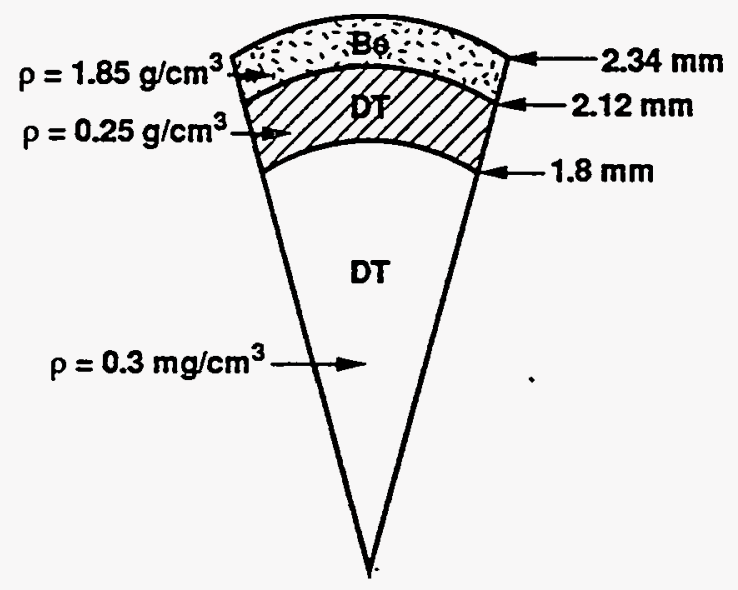

$50-01-00204-3165 p 001$

Figure 4-2. Fuel capsule structure.

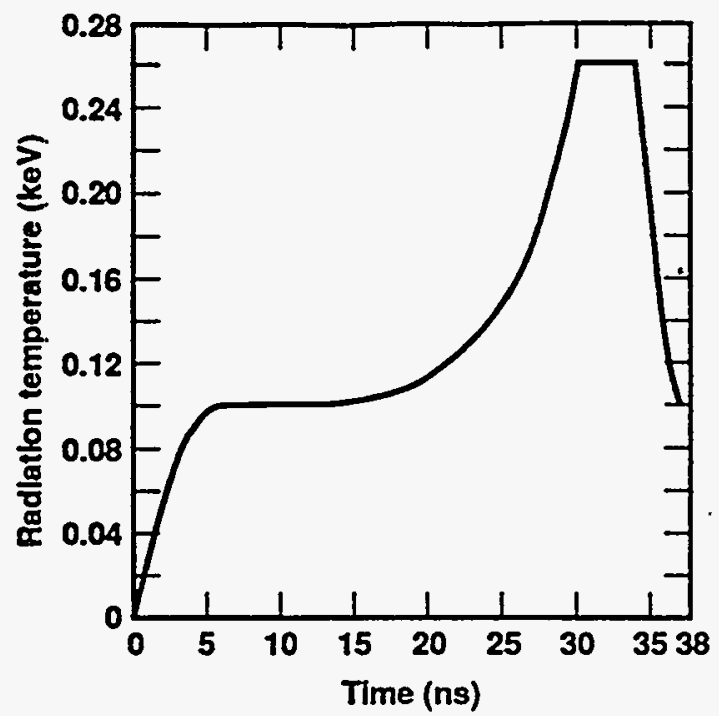

50-01-0894-3164pbo1

160Nathon

Figure 4-3. Hohlraum radiation temperature profile required for the implosion.

calculations, we expect that similar performance can be achieved with cheaper doped-plastic materials in future designs for lower-cost IFE target fabrication. For a capsule that absorbs about $1 \mathrm{MJ}$ and has an initial radius of $2.34 \mathrm{~mm}$, the minimum initial capsule-to-hohlraum surface area ratio that can provide an adequate timedependent symmetry requirement for the implosion is about 0.075 , based on calculations using the view-factor code GERTIE. ${ }^{3}$

Figure 4-4 shows the resulting timedependent soft $x$-ray symmetry on the capsule in terms of the ratio of amplitudes $A_{2} / A_{0}$ and $A_{4} / A_{0}$, which are the coefficients of the corresponding Legendre polynomials. This design has an overall energy coupling efficiency between the hohlraum and the capsule of $21 \%$ and a target energy gain of 80 . With an expected heavy ion accelerator efficiency in the range of 30 to $40 \%$, this target gain provides a product of driver efficiency and target gain of 24 to 32 , which is more than adequate for an IFE driver recirculating power fraction of less than $10 \%$. Future heavy-ion target designs can be better optimized for even higher gain. 


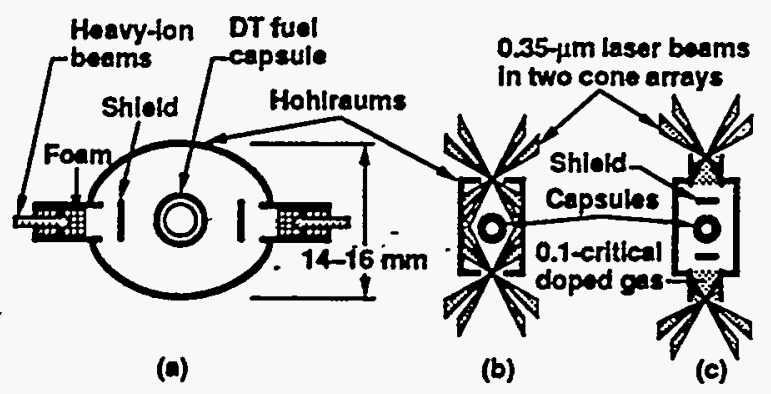

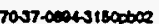

Figure 4-4. Schematic cross-sections of indirectdrive targets for (a) heavy ion power plant, (b) ignition test design for the NIF, and (c) a NIF model simulating the geometry of (a).

\subsection{The Use of the NIF to Validate Heavy Ion Target Designs for IFE}

In principle, heavy ion beams can be used for direct drive and indirect-drive targets. However, most conceptual studies of HIF power plants ${ }^{4-6}$ have selected indirect drive because of the greater complexity and cost of beam transport, and because of fusion-chamber interface associated with the larger number of beams and spherical illumination geometry required for direct drive. Thus we will limit this discussion to the use of the NIF to study indirect-drive HIF target physics. Figure 4-4 shows side-by-side crosssections of indirect-drive targets representative of the heavy ion-driven target design described in Section 4.2 [Fig. 4-4 (a)], the reference design for the NIF ignition target [Fig.4-4 (b)], and a conceptual NIF-HIF model target [Fig. 4-4 (c)] to better simulate the HIF hohlraum geometry of Fig. 4-4 (a).

Table 4-1 below compares some of the major target physics parameters of the heavy-ion (IFE) and laser ignition (NIF) targets of Fig. 4-4 (a) and 4-4 (b), respectively.

Table 4-1 shows that to demonstrate ignition, the NIF will test a higher capsule convergence ratio and a higher in-flight aspect ratio than required for HIF, both of those parameters indicate that NIF will demonstrate control of capsule hydrodynamic stability and fuel mix to a degree greater than HIF requires. Capsule implosion physics driven by soft $x$-rays in an indirect-drive hohlraum at a given hohlraum radiation temperature does not depend on the source that generated the soft $x$ rays, as long as the proper $x$-ray symmetry driving the capsule is maintained. In addition, the NIF target will require a higher hohlraum temperature and implosion velocity compared to HIF. The high temperature and high implosion velocity tend to increase hohlraum plasma generation affecting soft-ray transport symmetry within the hohlraum. In this respect also, the NIF will demonstrate control of hohlraum plasma effects on $x$-ray transport and drive symmetry to a degree greater than required for HIF. Thus, a successful ignition demonstration in the NIF will prove the feasibility of solving the most important and most difficult target physics requirements for HIF.

In addition to demonstrating the most important capsule implosion and hohlraum $x$-ray transport physics, the NIF can also be used to test targets such as depicted in Fig. 4-4 (c) designed to simulate the HIF target hohlraum geometry with greater fidelity, to further optimize HIF target design. The NIF will employ 192 laser beams illuminating the NIF target [Fig. 4-4 (b)] in two cones with different angles as shown in Fig. 4-4 (b). This allows experimental flexibility in controlling $\mathrm{P}_{2}$ and $\mathrm{P}_{4}$ soft $x$-ray asymmetries

Table 4-1. Comparison of target parameters for HIF and NIF.

\begin{tabular}{lcc}
\hline & $\begin{array}{c}\text { (a) HIF } \\
\text { target }\end{array}$ & $\begin{array}{c}\text { (b) NIF } \\
\text { target }\end{array}$ \\
\hline \hline Driver energy (MJ) & $4-6$ & 1.8 \\
Yield (M]) & $300-450$ & 15 \\
Convergence ratio & 27 & 36 \\
In-flight aspect ratio & 40 & 45 \\
Imploded speed & 32 & 41 \\
(cm/ $/ \mu$ ) & & \\
Maximum density, & $6.5 \times 10^{5}$ & $1.2 \times 10^{6}$ \\
$\rho\left(\right.$ (kg/m $\left.{ }^{3}\right)$ &. & \\
Maximum & 260 & 300 \\
hohlraum & & \\
temperature $(\mathrm{eV})$ & & \\
\hline
\end{tabular}


through different power and pulse shape control of the beams on the two cones. While this flexibility is important in a research facility such as NIF, IFE power plants designers would like to simplify target illumination requirements to minimize the number and separation of fusion chamber penetrations for driver beams.

Typically, HIF targets such as shown in Fig. 4-4

(a) are designed to be illuminated with only 10 to 20 ion beamlets from each end, clustered into a small cones of about 10 degrees around the target axis. Since the soft $x$-ray intensity radiating out of the beam converter sources on the poles, viewed from the capsule, would be higher than the $x$-ray flux from the equator of the hohlraum, "shine shields" such as shown in Fig. 4-4 (a) are typically invoked in HIF targets to improve HIF capsule x-ray drive symmetry. Recent NOVA experiments with shine-shields inserted into indirect-drive laser targets have shown that such shine shields can be used to control the $x$-ray drive symmetry on the capsule?

Recent studies ${ }^{1}$ have found that high- $Z$ plasma blow-off from the HIF hohlraum walls into the channels between the shine shields and the beryllium converters can significantly impede the transport of soft $x$-rays through those channels, and thereby affect the capsule implosion symmetry. A NIF-HIF-model target as shown in Fig. 4-4 (c) could employ two "gas bag" radiators driven by the NIF lasers to simulate the beryllium converter sources of the HIF targets. Gas bags are thin plastic bags filled with dense gas (e.g., $1 \mathrm{~atm}$ of neopentane at $\sim 10 \%$ critical density) so that the laser light can effectively absorb into and heat a resulting volume of plasma. The hydrocarbon gas can be doped with a high- $Z$ gas like xenon to provide similar radiation optical depth as that provided by the $\mathrm{Pb}$ dopant used in the beryllium converters of the HIF target. Recent success in volume heating dense gas bag targets on the NOVA laser facility at LLNL supports the suggestion that such gas radiators could be used to simulate heavy ion converters. ${ }^{8}$ These type of HIF-model targets can and should be first tested in NOVA at $\sim 1-n s$ pulse lengths. But such targets could also be tested in NIF at longer pulse lengths up to 10 to $20 \mathrm{~ns}$ more relevant to the HIF designs, where wall plasma blow-off can be more important. Such experiments in NIF can allow high-fidelity tests of ways to mitigate the effects of hohiraum wall blow-off for HIF targets by optimizing the hohiraum geometry and shine shields in a sequence of target modifications and tests. .

\section{References}

1. D. D.-M. Ho, J. A. Harte, M. Tabak, "Radiation-Driven Targets for Heavy-Ion Fusion," Proc. of 15th IAEA Int. Conf. on Plasma Phys. and Controlled Fusion Research (Seville, Spain, Sept. 6 to Oct. 1, 1994) IAEA CN-60/B-P-13 (1994).

2. R. W Petzoldt and R. W. Moir, "Target Injection Methods for Inertial Fusion Energy," Fusion Technol., 26, 896 (1994).

3. R. C. Kirkpatrick, Los Alamos National Laboratory Report No. LA-UR-86-1038 (1986); G. B. Zimmerman, Lawrence Livermore National Laboratory, personal communication (1993).

4. W. R. Meier et al., "OSIRIS and SOMBRERO Inertial Fusion Power Plant Designs," W. J. Schafer Associates Report, WJSA-92-01, DOE/ER/54100-1 (March 1992). Also see W. R. Meier, "OSIRIS and SOMBRERO Inertial Fusion Power Plant Designs - Summary, Conclusions and Recommendations," Fusion Eng. and Design, 25, 145 (1994).

5. L. M. Waganer et al., "Inertial Fusion Energy Reactor Designs Prometheus- $\mathrm{L}$ and Prometheus-H, Final Report," McDonnell Douglas, MDC 92E0008, DOE/ER-54101 (March, 1992).

6. R. W. Moir et al., "HYLIFE-II: A Molten-Salt Inertial Fusion Energy Power Plant Design Final Report," Fusion Technol., 25, 5 (1994).

7. T. J. Murphy and P. Amendt " $X$-ray Flux Symmetry Control in Advanced Nova Hohlraums," LLNL ICF Quarterly Report, 4, No. 3 (1994).

8. B. J. MacGowan, "The Study of Parametric Instabilities in NIF-Scale Plasmas on Nova," Proc. of 15th IAEA Int. Conf. on Plasma Phys. and Controlled Fusion Research (Seville, Spain, Sept. 25-Oct. 1, 1994) IAEA-CN-60/BP16 (1994). 


\subsection{The Role of NIF Within an Overall IFE Development Plan}

\subsection{A Long-Range IFE Development Plan Based on Defense Programs and Energy Research Cooperation}

\subsubsection{Introduction}

In preceding Sections 2,3 , and 4, we have described many important and essential ways the NIF would contribute to IFE development. In planning the rest of IFE development, it is important to consider the timing and relative costs of these NIF contributions within an overall IFE development plan, which includes the IFE driver development program elements, driver development activities carried out by Defense Programs (DP) in support of the Laboratory Microfusion Facility (LMF), ${ }^{1}$ and other needed IFE technology development activities which are not completely addressed by the NIF (e.g., lowcost target fabrication, high repetition-rate target injection and tracking systems, and fusion chamber technologies including IFE materials development).

This Section describes the role of the NIF in the context of a long-range IFE development plan for the next 30 years; it includes IFE developments needed for a solid basis to proceed with an Engineering Test Facility (ETF) decision by the time the NIF demonstrates ignition. The plan also includes the cost and schedule for the following tasks over 15 years: 1) construct the ETF facility, 2) conduct the ETF test program, and 3) construct an IFE DEMO to demonstrate net power production by 2025 . This preliminary plan, equivalent to a Work Breakdown Structure (WBS) Level-one plan, covers 1) Defense Programs R\&D which is relevant (dual-benefit) to IFE such as the NIF, and 2) IFE-specific R\&D. The
IFE-specific R\&D would need to be funded by Energy Research (ER) and by US industry and utilities for DEMO. This would be done at a pace sufficient to support an ETF decision by the time ignition is achieved with the NIF, and at a pace sufficient to achieve net power demonstration in a DEMO by 2025.

To capitalize on the success of fusion ignition in the NIF (expected around 2005), IFE development will need an Engineering Test Facility to test needed fusion power plant technologies, as called for in the 1990 Fusion Policy Advisory Committee ${ }^{2}$ and 1992 National Energy Policy Act ${ }^{3}$ plans. However, a decision to move forward with an ETF in 2005 also depends on the timely demonstration of feasibility for an efficient, high-repetition IFE driver. DOE's Energy Research Program is currently supporting heavy ion accelerator development as a candidate IFE driver to support the ETF decision.

Quoting from the recommendations ${ }^{4}$ of the 1993 Fusion Energy Advisory Committee Chairman Dr. Robert Conn to then Energy Resource Director Dr. Will Happer: "We recognize the great opportunity for fusion development afforded the DOE by a modest heavy-ion driver program that leverages off the extensive target program being conducted by Defense Programs. Consequently, we urge the DOE to reexamine its many programs, both inside and outside of Energy Research, with the view to embark more realistically on a heavy ion program. Such a program would have the ILSE (Induction Linac Systems Experiment) as a centerpiece, and be done in coordination with the program to demonstrate ignition and gain by Defense Programs."

Figure 5.1-1 shows the various existing and proposed major IFE development elements in an integrated plan, reflecting both DP (red for dual- 


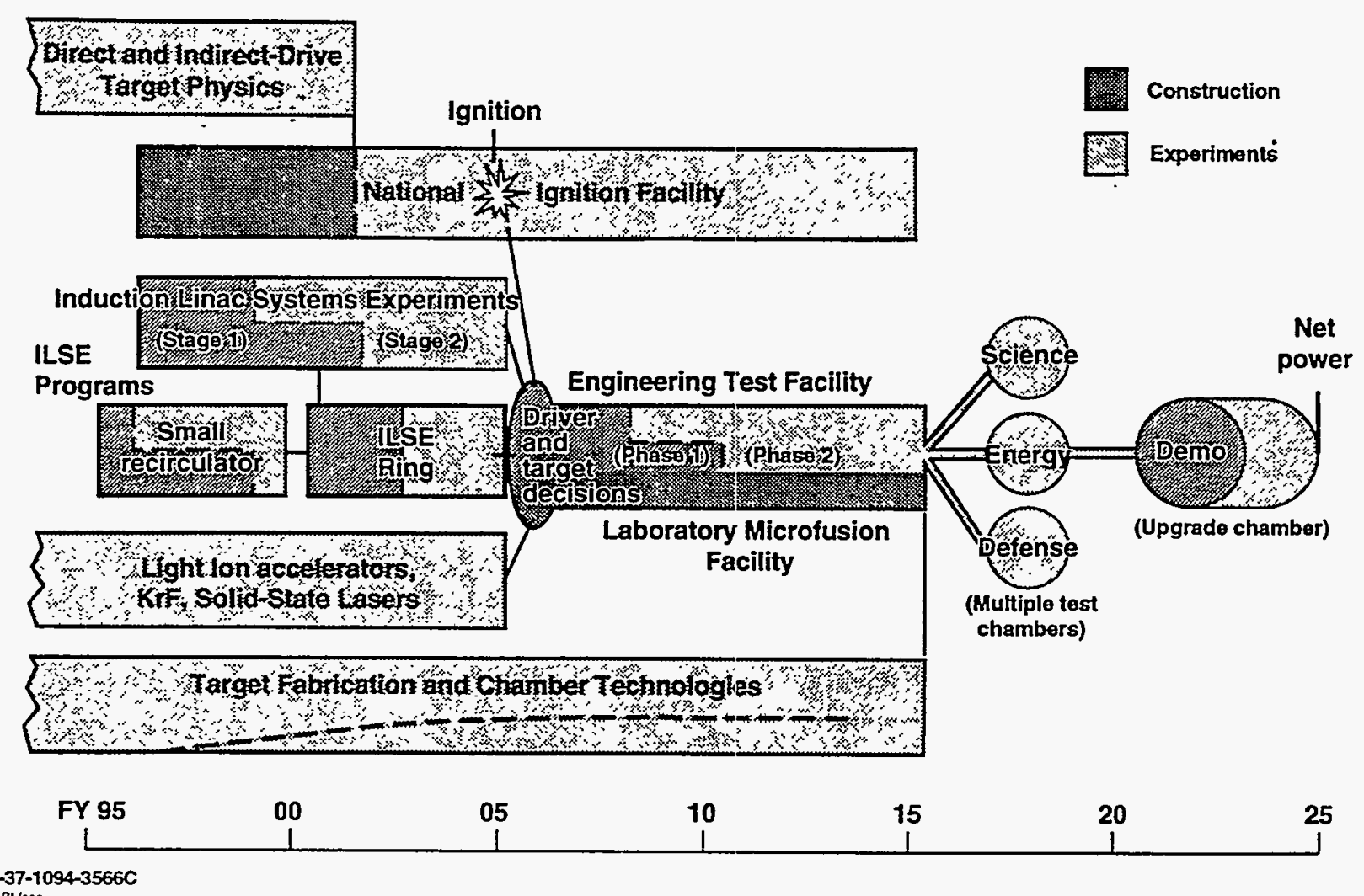

70-37-1094-3566C

Figure 5.1-1. An ICF/IFE long-range development plan to a DEMO with major R\&D elements of both DOE Defense Programs (red), and Energy Research (blue). Ignition (NIF) and IFE driver feasibility (ILSE) are major requirements for an ETF/LMF decision in 2005. A combined ETF/LMF with a common-staged driver is upgraded to a DEMO by adding an appropriate chamber in 2020. The DEMO is expected to be substantially supported and operated by US industries and utilities. This plan is a synthesis of Fig. 5.1-2 (ER-IFE plan) and Fig. 5.1-3 (DP-ICF plan).

benefit activities relevant to both defense and IFE) and ER contributions (blue for IFE-specific activities). Figure 5.1-1 is consistent with the 1990 National Academy of Sciences (NAS) ${ }^{5}$ and Fusion Policy Advisory Committee (FPAC) ${ }^{2}$ reviews of inertial fusion programs, the DOE/DP Inertial Confinement Fusion Five-Year Program Plan, 6 the 1992 National Energy Policy Act of Congress, ${ }^{3}$ and the 1993 Fusion Energy Advisory Committee (FEAC) Panel Seven Review, ${ }^{4}$ with one exception. In Fig. 5.1-1, the ETF for IFE development and the LMF for defense applications are combined into a single ETF/LMF facility for cost saving reasons (see Section 5.3).
The NIF (DP) and ILSE7 (ER) are the two most critical elements needed to establish the basis for an ETF decision in 2005. Using a single driver, an ETF can then be designed to evaluate several fusion chamber technology alternates at reduced power, greatly reducing the potential cost of IFE development leading to a demonstration power plant. Many review committees, including the National Academy of Sciences, the Fusion Policy Advisory Committee, the Fusion Energy Advisory Committee, and the Inertial Confinement Fusion Advisory Committee, have endorsed this parallel approach to IFE development. This approach entails the DOE Defense Programs (using the NIF for fusion 
ignition and gain demonstration), and Energy

Research play complementary roles.

A combined DP and ER strategy for IFE

development provides cost effectiveness three ways:

1. Developing an IFE option for fusion energy does not have to bear the cost of DP's target physics program, including the NIF to demonstrate inertial fusion ignition. The primary mission of the NIF is for national security; its secondary mission is for energy and science.

2. Most DP and ER driver options, including heavy ions as the primary candidate driver for IFE, could in principle, support a combined ETF/LMF dual-use facility following the NIF by driving multiple-target chambers for defense and energy missions.

3. Other IFE-specific development needs (e.g., IFE target design, low-cost IFE target fabrication, and IFE fusion chamber technologies) can be performed concurrently in the large installed base of DP facilities. IFE drivers, targets, and fusion chamber technologies can be largely developed separately in parallel, until integrated tests in the ETF are required. In many cases such as in target fabrication and chamber technologies, ER can augment DP supported facilities to carry out IFEspecific extensions of ongoing DP efforts for ICF needs.

\subsubsection{Dual Benefit of DP's Target Physics and Ignition Program for IFE}

The 1990 NAS/FPAC ${ }^{2}$ and 1993 FEAC Panel Seven ${ }^{4}$ reviews recognized the unique opportunity that an IFE fusion energy option could be developed cost effectively, given the investment by DP-ICF program to pursue inertial fusion ignition. ER funding would only be needed to cover the additional IFE-specific needs (high-pulse-rate IFE driver development, and IFE target design, fabrication, and chamber technology R\&D). The recommended minimum ER funding for these additional IFE-specific needs was approximately $\$ 17-25 \mathrm{M} /$ yr over the 1990s. 2,4 The recommended ER funding is small compared to the current DP-ICF program now running at about $\$ 180 \mathrm{M} / \mathrm{yr}$. The recommended ER funding is also consistent with a more recent evaluation of the cost and schedule needs for IFE, which is discussed below. The most important technical element needed for IFE over the next decade-the demonstration of inertial fusion ignition and energy gain in the NIF-is understandably also the single most expensive element needed for IFE over that period, but the $\sim$ \$1B cost of NIF is justified primarily for ScienceBased Stockpile Stewardship, as a key part of U.S. national security.

The above-mentioned reviews recommended that the ER program should concentrate on heavy ions as the primary driver option for IFE, based on a) the similarity of heavy ion accelerators to the experience of existing high-energy physics accelerators demonstrating high efficiency, reliability, and high-pulse-rates, b) the expected compatibility of heavy ion final focus magnets with the IFE fusion chamber radiation environment, and c) the compatibility of heavyion drivers with indirect-drive as the baseline target design for ignition in the NIF. However, the 1990 FPAC recognized that other driver programs supported by DP could also be backup options for IFE drivers. The DP program continues development of other driver options for the LMF high-yield defense mission, ${ }^{1}$ namely, solid-state lasers, $\mathrm{KrF}$ gas lasers, and light ion pulsed-power accelerators. DP recognizes heavy ion accelerators as another candidate driver for the LMF. Section 5.5 describes an advanced solidstate laser pumped by solid-state diode laser arrays, which can build upon the NIF development of multipass regenerative amplifier architecture. ${ }^{8}$

The solid-state and $\mathrm{KrF}$ laser options also support the possibility of direct drive for defense and IFE missions. ${ }^{*}$ Carrying the DP/ER cooperation theme a step further, Fig. 5.1-1

\footnotetext{
* While indirect drive is the preferred target type for heavy ions, direct drive is also possible with a sufficient number of heavy ion beams. Direct-drive target performance advantages may not outweigh the target chamber complexity required for a larger number of beams in direct drive for IFE, but NIF will provide the ultimate data for such a choice. Solidstate, diode-pumped lasers with an architecture similar to NIF, could become a desirable IFE driver candidate if diode costs are reduced sufficiently, and if target gains over 100 can be achieved. 8
} 
suggests that if a suitable driver can be found for both high gain and pulse rate, a combined ETF/LMF could be considered for both energy and defense missions. While indirect-drive is the highest confidence approach to ignition in NIF, $\mathrm{DP}$ also maintains a strong target physics program in direct drive, principally the OmegaUpgrade ${ }^{6}$ program, which is aimed at providing the basis for demonstrating high gain in the NIF. High gain is important for both defense and energy missions, and DP recognizes the importance of maintaining both direct- and indirect-drive options until the NIF confirms the target physics performance of both options. Thus, driver and target R\&D supported by DP and ER, taken together, allows a diversity of technical options to be kept open until the major decisions for the LMF and ETF need to be made.

To provide a concrete and specific ETF example for this overall plan, we assumed an ETF concept based on the heavy ion driver option described in the LMF Phase II Report, ${ }^{1}$ assuming that option would be selected at the $\sim 2005$ ETF decision point in Fig. 5.1-1. While we recognize that Defense Programs (ICF) supports development of other driver options for the LMF (i.e., solid-state lasers, light ion drivers and $\mathrm{KrF}$ lasers), we have chosen to use the HI driver in this example for the following reasons: (1) it is capable of meeting the requirements of both the LMF and IFE applications, (2) it was recommended by the National Academy of Sciences as the prime candidate for an IFE driver, and (3) it is the focus of the IFE driver program ${ }^{4}$ currently being funded by Energy Research within DOE.

We note, however, that the light ion pulsedpower technology is evolving towards modular induction-core systems with much similarity to that needed for heavy ions; there are many common development issues in light and heavy ion targets and beam transport in the chamber. Thus, we recognize the potential contributions of Defense Program's Light Ion program ${ }^{6}$ to the IFE driver development neẹds for a heavy-ion ETF option. Section 5.3 details the possible benefits of a more coordinated DP/ER development program, possibly leading to an improved common ion driver program for both LMF and
ETF missions. Assuming success of that combined development program, we envision an ETF facility in this IFE plan that, through nultiple target chambers, could in principle drive both an LMF chamber for defense applications as well as chambers to test IFE concepts. We assume, in adopting the heavy ion option for the ETF in this plan, that any common ion driver option in the range from light to heavy-ions would be at least consistent (or cost less) than the heavy-ion baseline. Keep in mind, also, we include both heavy ion driver development costs supported by ER over the next 10 years within the "IFE-specific (i.e., non-ICF)" costs in this plan, and the ICF driver development activities supported by Defense Programs, up until the target and driver decision point indicated in Fig. 5.1-1.

Finally, we note that the heavy ion driver option for this ETF plan is based upon the 5-MJ linear induction accelerator design described in the LMF Phase II study. ${ }^{1}$ At this point for planning purposes, we prefer to be conservative. The discussion in Section 5.2 will show that it will be desirable to conduct reduced-scale fusion chamber technology tests at low yields of 30-40 M], which may only require a driver energy of $2 \mathrm{MJ}$. While heavy ion target performance projections after the first NIF ignition experience may indeed support such a reduced ETF driver energy as an initial, upgradable phase of the facility, we cost the ETF driver now at the conservative level of $5 \mathrm{MJ}$, to preserve the high-yield ( $400 \mathrm{MJ}$ ) capability for the Defense LMF mission. Even at 5-MJ driver energy, other driver options for an ETF, such as a recirculating induction accelerator, may turn out to be less costly than a straight linac, but the other driver options are less-well understood at this time. However, we do include both linear and recirculating heavy ion driver developments prior to the $\mathbf{2 0 0 5}$ decision, since both are part of the ILSE program (the small recirculator experiments contribute to long-beam-path issues common to both linear and circular options, and the full ILSE program plan includes an ILSE ring ${ }^{4}$ following linear ILSE experiments).

The NIF ignition experiments will determine the most important and fundamental target 
physics needed to predict, with confidence, the minimum driver energy for ignition, and the scaling of energy gain with driver energy for a variety of possible IFE driver and target options. ${ }^{9}$ Section 4 described how:NIF data can provide confidence in the performance of heavy ion targets for the ETF and for future IFE power plants, apart from the issue of heavy ion beam focal spot size, which is addressed by the ILSE and other facilities.

\subsubsection{A Dual-Use ETF/LMF Following the NIF}

Figure 5.1-2, taken from the 1993 FEAC Panel Seven review, ${ }^{4}$ along with Figure 5.1-3 from the DOE-DP ICF 5-Year Program Plan, ${ }^{6}$ identify two separate facilities to follow ignition demonstration in the NIF: 1) the LMF for highyield defense applications, and 2) the ETF for developing high rep-rate target and chamber technologies needed for IFE. Both facilities would first require confirmation of ignition in the NIF. The nominal target yield for the LMF applications ${ }^{1}$ is similar to the typical yields that are found to be optimum in IFE power plant studies, namely, a few hundred MJ. The primary difference in LMF and ETF driver requirements is that an LMF mission can be satisfied with a fewshot-per-day capability, while the ETF mission requires testing at pulse-repetition rates of $5 \mathrm{~Hz}$ typical of IFE power plant requirements.

Heavy ion accelerators are the leading candidate driver for IFE in terms of efficiency and pulse rate capability, so that a 5-MJ heavy ion driver option should meet the mission requirements for both the LMF and the ETF. Furthermore, several heavy ion beam power plant studies have shown that a heavy ion driver cost is not a strong function of the driver pulse rate; the studies have also shown plausible ways

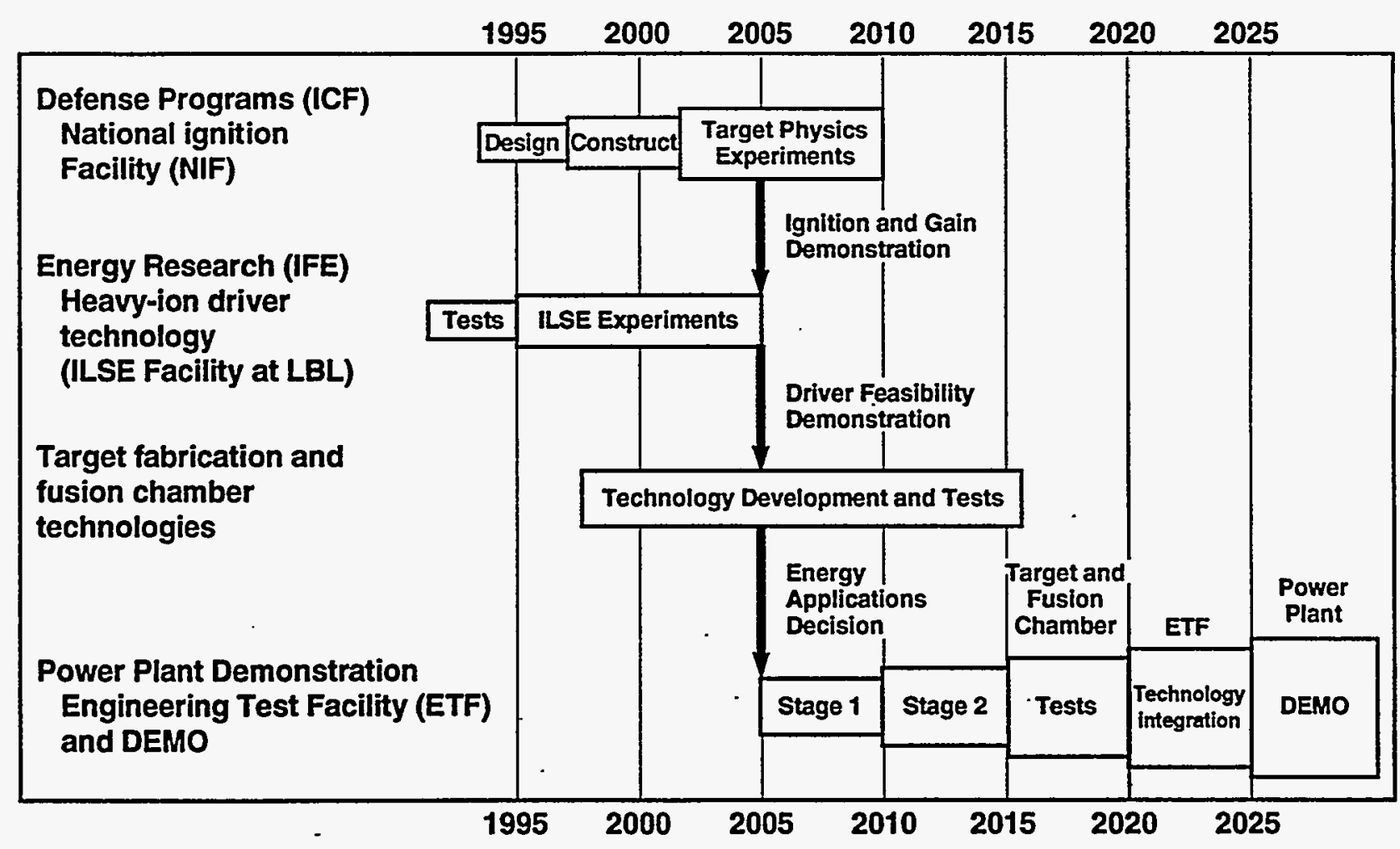

$05-00-0694-2746 \mathrm{pb01}$

Figure 5.1-2. The IFE development plan for DOE Energy Research as considered by FPAC ${ }^{2}$ and FEAC. 4 


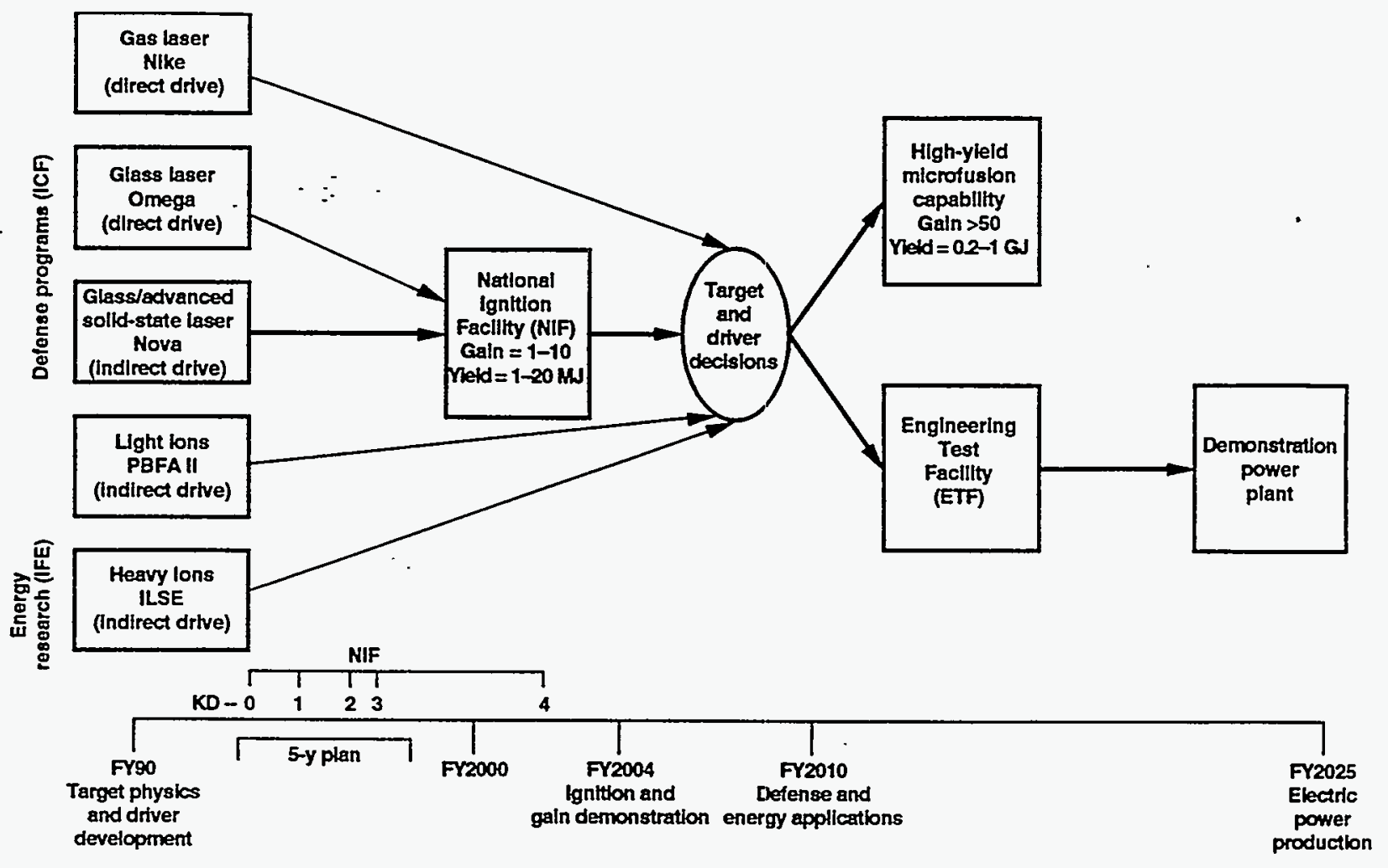

The NIF is being considered a key facility for stockpile stewardship

$70-00-0593-19000$

2/23/95

Figure 5.1-3. The DP-ICF program plan. ${ }^{6}$

for a heavy-ion beam to be switched with magnets to allow one driver for several target chambers. ${ }^{10-11}$ This raises the possibility that a single heavy ion driver might be used to drive both ETF and LMF target chambers. The different target chamber test environments required for the defense and energy missions could be accommodated with different target chambers. Other driver options supported by DP may, if successful, lead to lower cost for a combined ETF/LMF than a heavy ion driver, or, in the case of laser drivers, may be better able to exploit direct drive, should direct drive prove to have sufficiently higher gain than indirect drive (the latter possibility would require demonstration of better control of hydrodynamic instabilities associated with higher aspect-ratio direct-drive capsules). Light ion drivers need to couple to small-radius target chambers, so that separate target chambers for ETF and LMF experiments might be successively moved into place for a light-ion driver (see Section 5.3). Laser driver options could be switched to fixed multiple target chambers by using suitable Pockels Cell arrays. ${ }^{12}$ Thus, all DP and ER driver options indicated in Fig. 5.1-1 could in principle, support a combined ETF/LMF. However, an important caveat needs to be recognized: if a) only single-shot-capable driver options were ready at the 2005 decision point, and b) defense programs had a pressing need to expedite the LMF, then separate LMF and ETF facilities would be forced due to delay in the high pulse-rate ETF.

Since the costs for either LMF or ETF test chambers are expected to be a small fraction of the driver cost, a combined ETF/LMF should result in substantial cost savings compared to two separate facilities. Section 5.2 shows that development of several IFE fusion chamber technology options might be expedited through 
the use of multiple target chambers, and can be tested at reduced scale ( $<1-2-\mathrm{m}$ radius chambers) and at low yields ( $<40 \mathrm{MJ}$ ) with $\sim 5-\mathrm{Hz}$ pulse rates, so that the average fusion power and associated power and-tritium handling costs can be minimized. Since the driver cost to produce $400 \mathrm{MJ}$ yield is typically more than $2 / 3$ of the cost of a driver capable of producing $400 \mathrm{MJ}$ (because of the gain curve and economy-scale effects), the driver cost increase to provide $5 \mathrm{MJ}$ for a highyield LMF should be less than $40 \%$, compared to a 2-MJ driver needed to provide initially lower yields for low power ETF testing. A 5-MJ driver would also provide more design margin to cover target gain uncertainties. Another benefit of an ETF/LMF driver having both high-yield and reprate capability would be that once a satisfactory fusion chamber technology is identified and qualified in the ETF stage at low-average fusion power, an IFE DEMO could then be obtained by scaling up the chosen fusion chamber to be capable of 200-400-MJ yield at a few $\mathrm{Hz}$ to provide enough average power and gain for a confident net electric power demonstration. This would reduce the DEMO-specific add-on costs, thereby facilitating US industry and utility support for the DEMO.

To summarize, Fig. 5.1-1 depicts a new strategy for a combined LMF/ETF sharing a common driver capable of both the LMF and ETF missions, and with separate target chambers optimized for each mission. The LMF mission is to provide sufficient ICF yield to extend weapons physics and NWES capabilities beyond those of the NIF. The ETF mission is to develop IFE fusion chamber technology and driver performance and reliability at high rep-rates representative of an IFE power plant. The goals of this combined DP and ER strategy are:

1. Increase total DOE resources for driver technology development for both LMF and ETF through cooperation by DP and ER on elements leading to a common driver. ER could continue to concentrate on the heavy ion driver option as at present.

2 Reduce the total cost of accomplishing the LMF and ETF missions through a combined facility sharing a common driver, compared to two separate facilities.
3. Increase motivation for the next inertial fusion facility after NIF by offering more diversity for its use and benefits, as in the case of NIF.

It is important to note that there are many common elements between DP's light ion program and ER's heavy ion program in the areas of indirect-drive target physics, highcurrent ion beam transport and focusing, and high-pulse rate induction accelerator technology. Cooperation between these two programs is a good example of possible synergism between DP and ER for the mutual benefit of both programs. The prime motivation for this new strategy is to make the next step after NIF both more affordable and desirable, and to increase the likelihood of having a driver technology ready to support a decision for that new facility by the time ignition is achieved in the NIF.

\subsubsection{DP-ER Cooperation in Low-Cost IFE Target Fabrication, and IFE Fusion Chamber R\&D}

Recent IFE power plant studies ${ }^{13-15}$ have outlined several critical R\&D areas essential to IFE success besides high-target gains and efficient, high-pulse rate drivers, including:

- Low-cost target fabrication methods suitable for large-scale mass production, with an understanding of the trade-off between target gain, the precision limits for those fabrication methods, and the cost per target.

- Long-life fusion chambers able to withstand $>3 \times 10^{8}$ pulses without replacement, and with rapid clearing of target debris to allow pulse rates of 5-10 Hz.

- Development and characterization of low-activation materials, primarily carbon-based composites, for those IFE chamber technologies requiring such materials exposed to substantial neutron fluences, and for damage effects on final beam optic elements, including, where appropriate, high-peak neutron dose rate effects.

Some progress in identifying possible solutions to these critical IFE areas may in fact be 
required to convince the technical committee that would be reviewing the readiness to proceed to an ETF/LMF prior to the 2005 decision point shown in Fig. 5.1-1. Here again, there are opportunities for DP-ER-cooperation for the mutual benefit of both programs:

- When DP completes its cryo-DT target fabrication program ${ }^{6}$ in support of Omega-Upgrade within the next few years, much of the most expensive infrastructure needed for testing any IFE fabrication method will be in place, including: a) tritium-qualified facilities for filling capsules, b) technology for producing smooth DT fuel layers, and c) extensive target diagnostic equipment for characterizing target precision specifications. Additional funding from ER for small-scale feasibility tests for low-cost IFE target fabrication and transport (injection and tracking) would benefit from utilizing the DP target fabrication infrastructure. DP would benefit if suitable and lower cost methods were found from the IFE tests (e.g., lower cost hohlraums for NIF, capsule filling techniques that reduce tritium inventories).

- DP will continue R\&D techniques to make more ablation- and shock-resistant walls and final optics, not only to accommodate the high-yield LMF chamber requirements, but also to permit NIF to explore the possibility of higher target gains that may prove to be feasible. The NIF target chamber dynamics experiments described in Section 3 will contribute critical data in this area. These DP efforts may be utilized in some IFE fusion chamber concepts (e.g., for direct drive). On the other hand, R\&D on IFE concepts utilizing sacrificial (renewable liquid) layers to protect critical inchamber components from soft $x$-ray ablation, could help develop analogs employing sacrificial frost layers to allow NIF or LMF to accommodate higher yields with less-frequent final optics replacements. Most importantly, both
DP- and ER-sponsored experiments in this area can jointly contribute important data needed to calibrate various radiation-hydro codes such as CONRAD ${ }^{16}$ and TSUNAMI, ${ }^{17}$ which are used to design both the NIF target área and several IFE fusion chamber concepts.

- DP has developed several pulsed neutron sources for defense needs (e.g., LLNL Physics Department's 100-MeV electron linac, SNLA's pulsed fission reactors, and a future upgrade of LAMPF called LANSCE at LANL) which can be used to provide critical data on neutron damage dose rate effects important to NIF and LMF (e.g., damage to fused silica, KDP crystals, and low-activation carbon composites used for in-chamber structures). These materials tests for DP can also be important for some IFE concepts (e.g;, for IFE concepts employing structures exposed to significant neutron fluences). Even more important to IFE, however, would be the utility of data on effects of dose rate to damage, to provide a critical and stressing test of nonlinear solid-state physics (overlap of damage cascades before annealing mechanisms can occur) in MDS codes. ${ }^{18}$ MDS codes, many of which are supported by ER in Basic Energy Sciences, may ultimately be able to predict the useful service life of candidate IFE materials needed in IFE candidate designs, reducing future IFE development time and cost. ${ }^{18}$ The same capability developed for ER would also benefit DP materials selection for critical NIF and LMF final optics and inchamber components.

\subsubsection{Overall Cost and Schedule for IFE Development}

Figures 5.1-4 and 5.1-5 present the overall IFE development cost and schedule in the form of two EXCEL spreadsheets: the first for IFE-specific development needs (those important to IFE but not required to carry out the Defense ICF mission), and the second for Defense-Program 


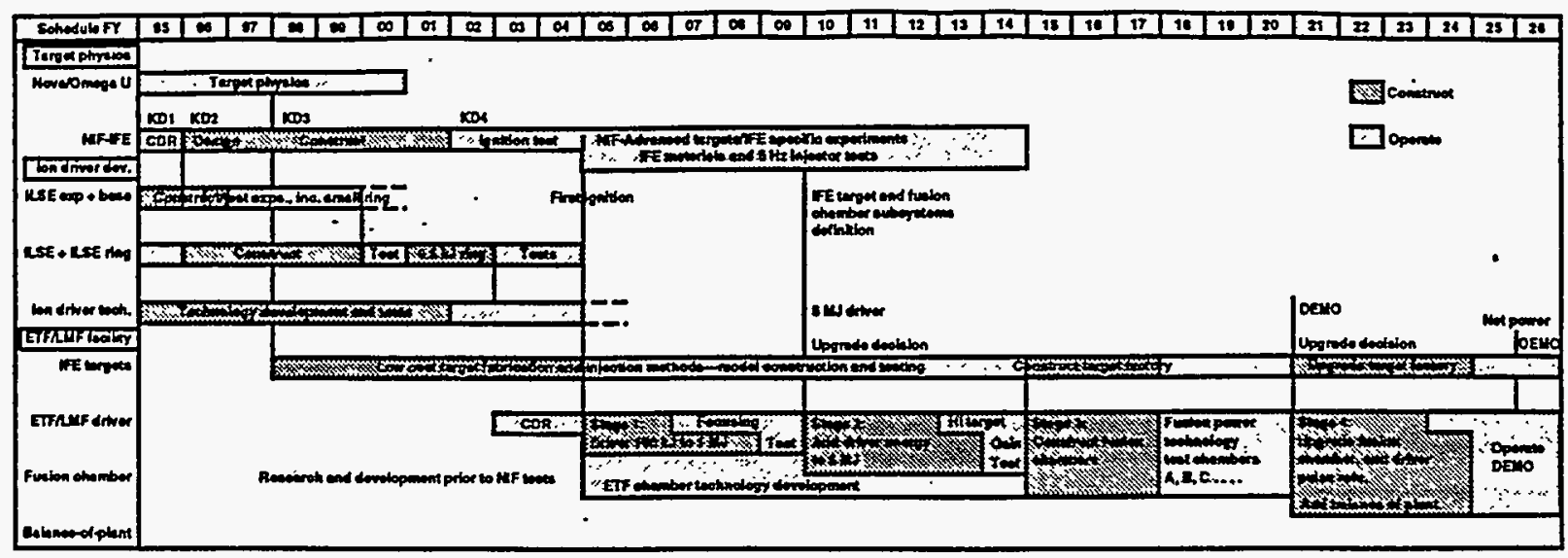

Costs for IFE and ETF facllty, apart from ICFNIF Ignition program and LUF (millions of constent FY95 doilars)

(Operating as woll as conatruetion S's. ETF driver cost besls $=$ twice 10 th-of-kind commercial estimatios $+20 \%$ contingency. Add $+10 \%$ uncertalnty to total IFE costs)

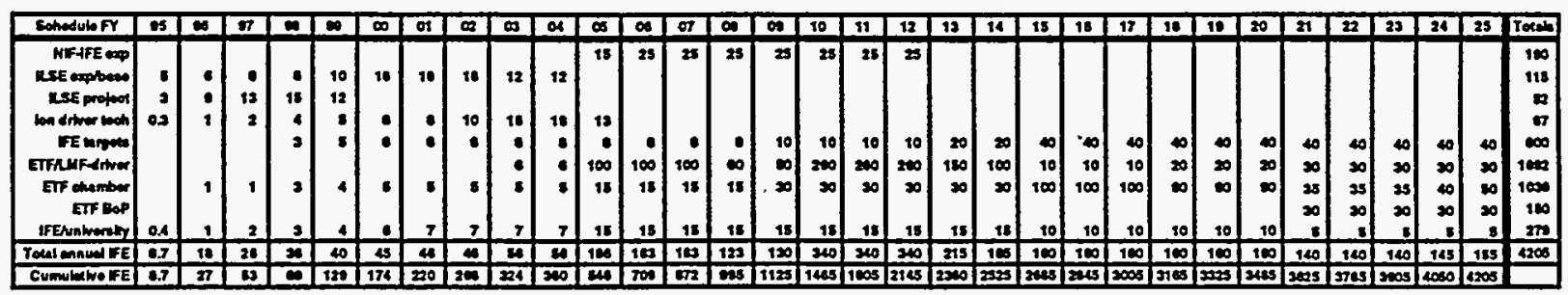

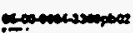

Figure 5.1-4. Cost and schedule for IFE-specific development needs (apart from R\&D covered by DPICF R\&D) out to net power demonstration. Costs are in constant 1995 dollars and include both construction and operating costs.

R\&D programs for Stockpile Stewardship, which are also relevant to IFE as dual-benefit. Both figures together support all the elements shown in the DP and ER strategic plan depicted in Fig. 5.1-1. The main elements are: the NIF, Induction Linac Systems Experiments, and a staged Engineering Test Facility/Laboratory Microfusion Facility with multiple-target chambers for rapid chamber development, test and selection. All costs are in constant FY95 dollars due to the long time scales and uncertainty in projecting effects of inflation over many years.

The 1992 National Energy Policy Act of Congress (EPACT) ${ }^{3}$ calls for the development of both MFE and IFE, setting a goal of a DEMO by 2025 for both programs. In this section, we estimate cost and schedule needs for all IFEspecific (ER and industry supported R\&D) and DP-IFE-relevant development activities to provide a DEMO, paced to meet the 2025 goal. These are cost and schedule needs; they do not necessarily reflect actual DOE ER or DP budget plans in all cases. Lower annual spending rates than estimated here imply a longer time to achieve an IFE DEMO. These estimates are provided for a few broad categories of $R \& D$, and so considerable uncertainty in these estimates exists until more detailed plans are developed. The uncertainty in the total integral costs are likely to be less than the uncertainty in any individual item.

The cost and schedule need for IFE development are summarized in Figs. 5.1-4 and 5.1-5 for IFE-specific and IFE-dual benefit activities. Since DP contributions to IFE are limited to areas of dual benefit to Science-Based Stockpile Stewardship defense R\&D and to IFE, we have separated IFE cost and schedule estimates into two spreadsheets: one for IFEspecific R\&D needs, which are not likely to be covered by defense programs, and one for dual benefit IFE-relevant parts of defense programs. Some readers will associate total IFE development costs to the IFE-specific costs, since the DP-IFE relevant costs are fully justified for 


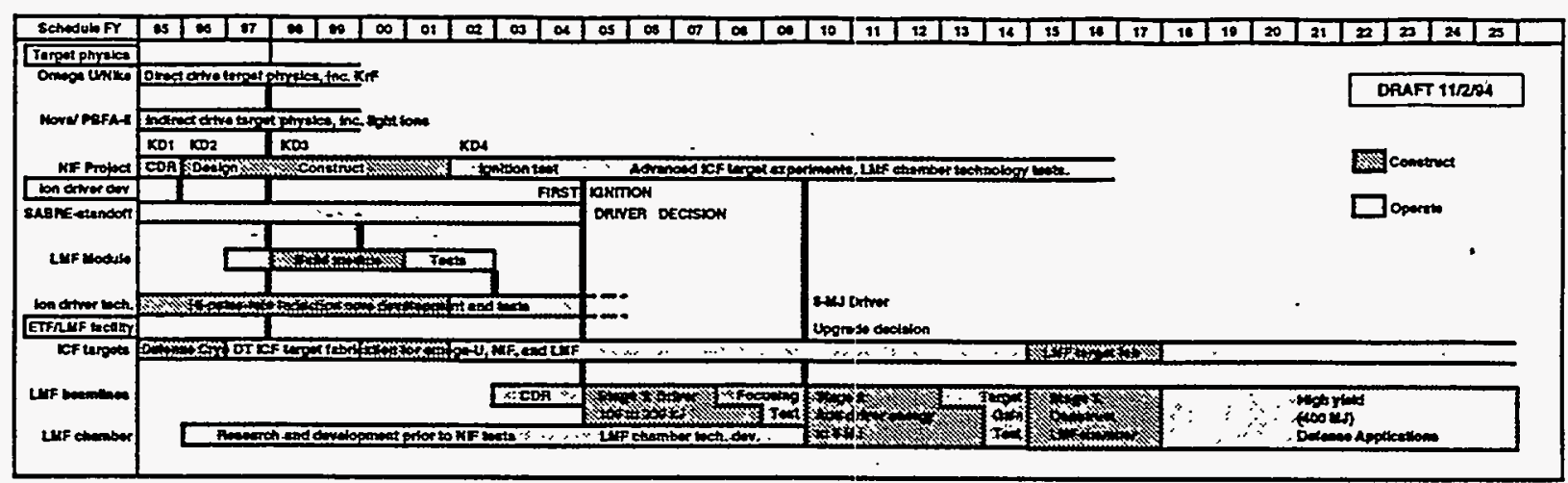

Costs for DP-ICF programs with dual-benefit for defense and energy missions (millions of constant FY95 dollars)

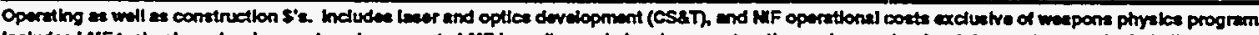

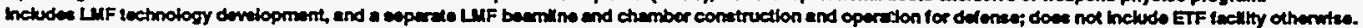

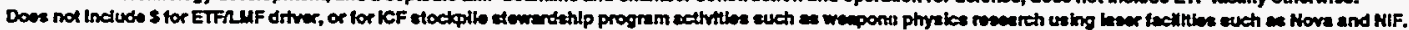

\begin{tabular}{|c|c|c|c|c|c|c|c|c|c|c|c|c|c|c|c|c|c|c|c|c|c|c|c|c|c|c|c|c|c|c|c|c|}
\hline Setheculo FY & is & $\infty$ & 97 & $\boldsymbol{\omega}$ & $\infty$ & $\infty$ & 01 & $\infty$ & $\infty$ & or & os & $\infty$ & or & $\infty$ & $\infty$ & 10 & 11 & 12 & 13 & 14 & 23 & 18 & 17 & 18 & 11 & 20 & 21 & 22 & 20 & 24 & 23 & Torat \\
\hline Omegentrike & 20 & 20 & 24 & 2 & 20 & 20 & & & & & & & & & & & & & & & & & & & & & & & & & & 10 \\
\hline NOTE PBFA of & 4 & $\infty 0$ & $\infty$ & $\infty$ & 25 & 28 & & & & & & & & & & & & & & & & & & & & & & & & & & 274 \\
\hline $\begin{array}{l}\text { MFF Proted } \\
\text { Mr Oparsition }\end{array}$ & 11 & $\omega$ & $\bullet$ & 14 & 200 & 178 & 100 & $\infty$ & $\infty$ & $\infty$ & $\infty$ & $\infty$ & $\infty$ & $\infty$ & $\infty$ & $\infty$ & $\infty$ & $\infty$ & $\infty$ & $\infty$ & 0 & $=0$ & & & & & & & & & & $m$ \\
\hline $\operatorname{css} x$ & es & $\infty$ & 25 & 5 & - & $s$ & - & & & & & & & & & & & & & $w$ & 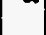 & $w$ & & & & & & & & & & 180 \\
\hline SABRE-2tendod & ? & 90 & t5 & $\infty$ & 20 & 20 & 20 & 20 & $\infty$ & 20 & & & & & & & & & & & & & & & & & & & & & & 17 \\
\hline LWF Mocuin & & & 10 & 88 & 25 & 25 & 15 & 10 & & & & & & & & & & & & & & & & & & & & & & & & 100 \\
\hline 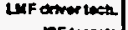 & I & 3 & - & 10 & 10 & 10 & 10 & 10 & * & 10 & & & & & & & & & & & & & & & & & & & & & & is \\
\hline CF taposed & 18 & 18 & is & n & 20 & 20 & 21 & $2 t$ & 2 & 22 & 2 & 22 & 22 & $\mathbf{2 n}$ & 22 & 2 & 2 & $\boldsymbol{n}$ & 2 & 2 & 26 & $\mathbf{2 8}$ & 25 & $\boldsymbol{\infty}$ & $\infty$ & 20 & 20 & 20 & $\mathbf{2}$ & $\mathbf{2}$ & $\mathbf{2 0}$ & as \\
\hline LFF bermismen & & & & & & & & & & & & & & & & & 10 & 10 & $x$ & 30 & $\boldsymbol{\infty}$ & $\boldsymbol{\infty}$ & $\infty$ & $\mathbf{\infty}$ & $\boldsymbol{\infty}$ & 20 & 20 & 20 & $\infty$ & $\mathbf{\infty}$ & 20 & 275 \\
\hline LuF chemoser & & 1 & 1 & 1 & 1 & 1 & 2 & 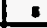 & 8 & 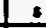 & 8 & 5 & 1 & 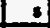 & 3 & 10 & 10 & 10 & to & 10 & 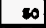 & 20 & $\infty$ & $\boldsymbol{\infty}$ & $\infty$ & $\infty$ & $\infty$ & $\infty$ & $\infty$ & $\infty$ & $\infty$ & $\boldsymbol{\omega O T}$ \\
\hline AgnUal DPAFE & 770 & 200 & 247 & 20 & 253 & 220 & 120 & 170 & 1197 & 197 & 107 & 107 & 18 & 17 & 18 & 17 & 1102 & 102 & 102 & 100 & 173 & in & 118 & $\infty$ & $\infty$ & $\infty$ & $\infty$ & $\infty$ & $\infty$ & $\infty$ & $\infty$ & 1000 \\
\hline Cumvleto DNA & 178 & $\mathbf{m 1}$ & 122 & ess & 1316 & 1603 & IDX & 2010 & 2121 & 24 & 2031 & 2416 & 2305 & 2392 & 2070 & $27 \pi 8$ & $20 \pi$ & 2000 & 300 & 314 & 2050 & 2534 & 2us & 3720 & 3000 & 2910 & 1000 & 4000 & 4100 & $\mathrm{~cm}$ & 408 & \\
\hline
\end{tabular}

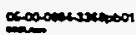

10min

Figure 5.1-5. Cost and schedule estimates for long-term Defense Programs ICF R\&D which is relevant (dual benefit) to IFE, including the LMF, out to 2025. All costs are in constant 1995 dollars and include both construction and operating costs.

national security alone; other readers will interpret total IFE development costs to be the total DP and ER costs. In some IFE development needs, it is not yet clear whether DP or ER should fund the indicated activity, hence the assignment of some items might change in the future.

Several major assumptions were made to allow a complete plan to a DEMO in Figs 5.1-4 and 5.1-5:

1. NIF construction start in FY96, and first ignition by 2005 .

2 Completion of all ILSE program elements by 2005.

3. A combined ETF/LMF facility is assumed with multiple-target chambers for defense and IFE technology tests, sharing a common driver. The ETF/LMF driver cost is arbitrarily included in the list of IFE-specific costs, even though it is used to drive an LMF dedicated target chamber and IFE test chambers.
4. A staged heavy ion linear induction accelerator with a beam switchyard is assumed for the ETF/LMF for costing. purposes as a conservative assumption; other driver candidates are less mature for this mission, but aim for lower costs.

5. US industry and utilities can obtain a DEMO by upgrading a suitable fusion chamber developed in the ETF test phase; the ETF/LMF site is assumed capable of a DEMO.

The cost and schedule estimates for the DP and ER development needs for IFE in Figs 5.1-1 and 5.1-2 are based upon:

1. Schedule and spending rates paced to provide a DEMO by 2025, as called for by the 1992 National Energy Policy Act of Congress, and to have all needed technical data to support a decision to proceed to an ETF by the time ignition is expected in the NIF ( 2005). 
2. NIF Conceptual Design Report ${ }^{19}$

3. ILSE/Elise Conceptual Design Report ${ }^{7}$

4. The FEAC Panel Seven Report and Recommendations $^{4}$

5. LMF Phase II Report ${ }^{1}$ plus the WJSA ITF Study Report ${ }^{20}$ used to provide conceptual basis of a combined ETF/LMF . All current DP and ER driver developments for the LMF and ETF are included up to 2005 decision date. The ETF/LMF driver cost is based upon a straight heavy ion linac option (provided by Ed Lee of LBL) for conservative total IFE development cost estimate.

6. Core Science and Technology Report, ${ }^{21}$ for base ICF technology development needs also relevant to IFE. (See Section 5.4)

7. The February 1994 national NIF-IFE workshop held at UC Berkeley ${ }^{9}$ (See Section 3 for description of the target fabrication and target systems elements considered. The costs were based on the current and planned-out year DP budgets for target fabrication and R\&D, and on a judgment of how much additional $R \& D$ was needed specifically for IFE, relative to DP R\&D).

Fig. 5.1-4 distributes all tasks into nine WBS Level-one cost categories:

1. NIF-IFE experiments. This covers the additional IFE-specific target experiments on the NIF described in the February 1994 NIF-IFE workshop (see Section 3.2) and further discussed in Section 4. The NIF-IFE experiments were costed as 3000 total shots for 5 years after first ignition, at a cost of $\$ 50 \mathrm{~K} / \mathrm{shot}+$ \$40M other.

2 ILSE exp/base. All ILSE program elements are represented in two parts: an experimental/base operating program (this item) and the ILSE project itself (the next item). ILSE Exp/Base includes linac theory and small experiments, small recirculator experiments (see Fig. 5.1-1), and later modifications of the ILSE facility, such as the follow-on ILSE-Ring. (See FEAC Panel Seven Report, Section 3.1 of Ref. 5)
3. ILSE Project. This would be the ILSE line-item project as proposed in the FEAC 7 report. This project may be phased, with the first phase to be called ELISE.

4. Ion driver technology. This covers the heavy ion driver technology and industry technology transfer recommended by the FEAC Panel Seven report. This technology development is needed in addition to the ILSE physics to support the ETF decision in 2005.

5. IFE targets. This item includes development of low-cost manufacturing methods for IFE targets, and for the highrepetition-rate target injection and tracking systems.

6. ETF/LMF driver. This item covers both the construction of a staged heavy ion linac driver for the ETF/LMF, and the costs of operating each accelerator phase to work-out the operational problems and achieve high-reliability $5-\mathrm{Hz}$ rep rates.

7. Fusion chamber. This includes the needed pre-ETF/LMF IFE reactor technology development activities, including NIF-related experiments described in Sections 3.3 and 3.4, as well as the target shooting test phases and multiple-fusion chamber technology test phases of the ETF part of the ETF/LMF program, and the final DEMO fusion chamber upgrade and operation to net power demonstration.

8. ETF BoP. This adds the steam balanceof-plant (BoP) to the end of the ETF test phase, to support the DEMO upgrade to net power demonstration.

9. IFE/University. This is intended to include small university programs supporting the above IFE development activities, as well as IFE systems studies by multilab, university and industry teams. Support for systems studies including universities, while a small fraction of the total IFE expenditures, 
is crucial to training the future generation who will carry out the ETF/LMF/DEMO research programs.

The categories 5 to 8 are chosen to reflect the respective preceding deyelopments needed to support the four basic parts of a generic IFE power plant, shown in Fig. 5.1-1. All nine categories cover all the FEAC Panel Seven recommended IFE development activities. In addition, this plan includes additional IFEspecific target experiments on the NIF in Category 1, as called for in the NIF-IFE workshop (Section 3.2), which FEAC Panel Seven did not include. The "Fusion chamber" and "IFE target" development costs 1998 to 2005 are very uncertain, and depend on the range of IFE chamber and target types to be developed for testing in the ETF/LMF chamber test sequence. The final DEMO stage, mainly an upgrade in rep rate and power handling/conversion, adds about $\$ 600 \mathrm{M}(\sim \$ 100 \mathrm{M}$ in accelerator front end power for higher rep-rate, $\sim \$ 200 \mathrm{M}$ for higher rep-rate target fabrication and injection systems, $\sim \$ 150 \mathrm{M}$ for a high rep-rate chamber upgrade and tritium recovery system, and $\sim \$ 150 \mathrm{M}$ for a $100-\mathrm{MWe}$ steam conversion plant). The $\$ 600 \mathrm{M}$ add-on costs for a DEMO upgrade are assumed to be provided by US industry and utilities, but is included in the "IFE-specific costs in Fig. 5.1-4. The assumption of an upgradable ion driver for the ETF allows the driver to be constructed in stages, so that most of the ETF facility cost with multiple target chambers need not be committed at the ETF Stage-1 start decision in 2005. However, it is assumed that the ETF site and Environmental Impact Statement are chosen to permit upgrades all the way to a final DEMO stage producing a small amount of electric power by 2025. One of the target chambers driven by a common ion driver is assumed to be used as an LMF to reduce total costs between DP and ER for both defense and energy missions (but LMF applications are not included in these "IFE costs"). This ETF/LMF strategy allows time for the IFE experiments using NIF to help define the ETF/LMF target systems and chamber design, while shortening the overall schedule to the DEMO compared to a sequence of separate facilities. This is critical in meeting the goal of a DEMO in 2025; a sequence of ILSE $\rightarrow$ IDF $\rightarrow$ ETF/LMF $\rightarrow$ DEMO separate facilities would take too long.

Figure 5.1-5 distributes all DP-IFE-relevant tasks into 11 WBS Level-one cost categories:

1. Omega Upgrade/Nike. This is the DP target physics programs for direct drive.

2 Nova/PBFA-II. This is the DP target physics program for indirect drive.

3. NIF Project. This is the construction cost [Total Project Cost (TPC)] of the NIF to demonstrate inertial fusion ignition and gain, testing both direct- and indirect-drive performance.

4. NIF Operation. This is the nominal NIF facility operation costs, not counting the specific research programs for weapons physics and Science-Based Stockpile Stewardship (SBSS). This operation, plus the NIF TPC, constitutes the $\$ 1.8 \mathrm{~B}$ NIF lifecycle cost.

5. CS\&T. This is the supporting base ICF program in Core Science and Technology, including laser and optics component development.

6. SABRE standoff. This is the SNL program to develop the transport and focusing of highcurrent ion beams to the target at a distance ("standoff"), using the multistage extraction diode facility SABRE.

7. LMF Module. This is the SNL pulsed-power module prototype for the LMF, based on "voltage-adder" induction accelerator technology.

8. LMF driver technology. This is the SNL high-average power, high pulse-rate induction module technology program (RHEPP facility).

9. ICF targets. This is the DP target fabrication and $R \& D$ program to develop cryogenic $D T$ targets for the Omega-Upgrade and the NIF.

10. LMF beamlines. This is the beam transport line from the common ETF/LMF driver to the LMF chamber.

11. LMF chamber. This is a large LMF chamber dedicated to high yield defense shots driven by the common ETF/LMF driver.

Figure 5.1-6 plots the combined annual spending rates consistent with Figs. 5.1-4 and 5.1-5. Construction costs for the NIF and for the 


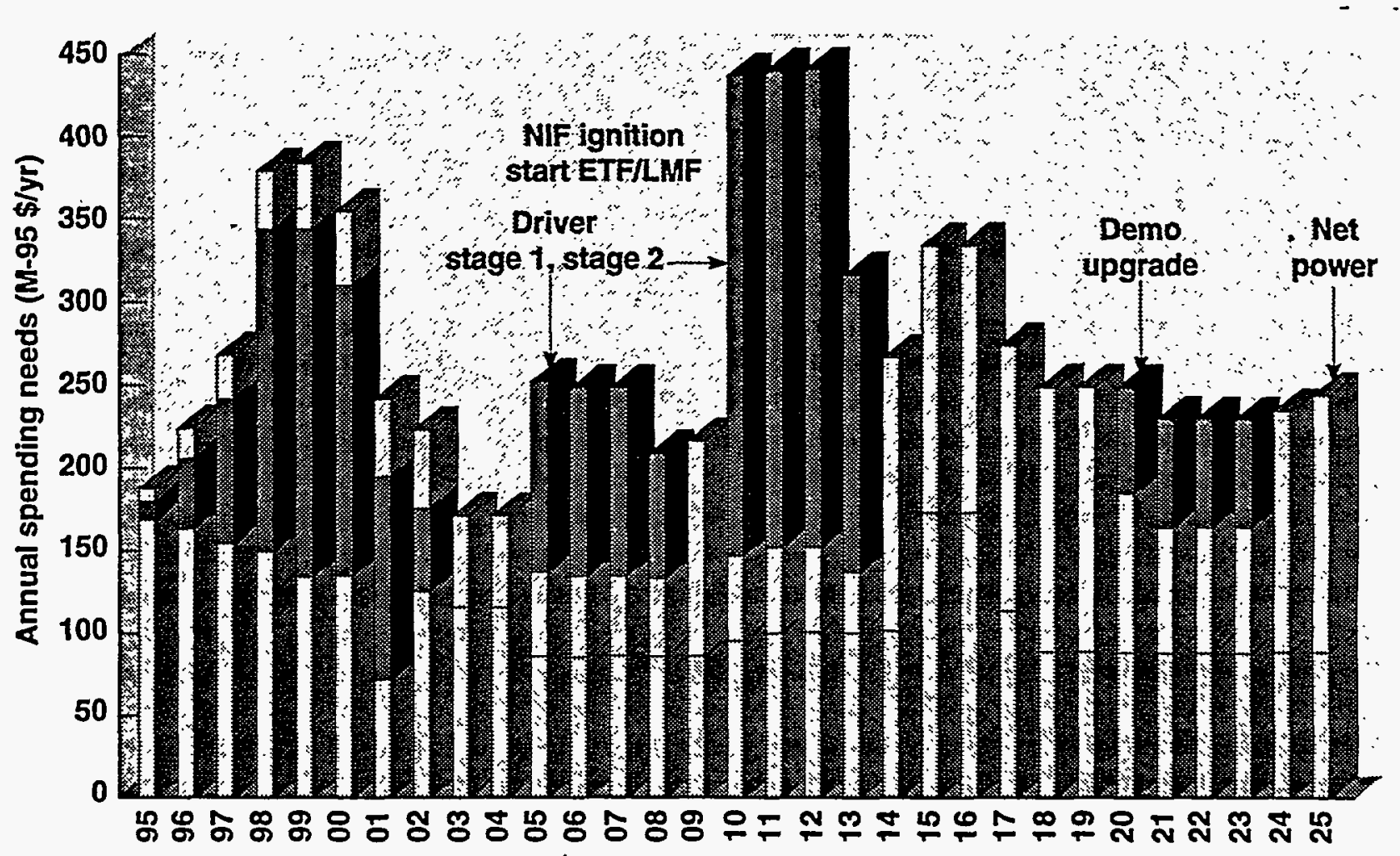

Fiscal year

05-00-1294-3939pb01

Figure 5.1-6. Annual costs for both IFE development R\&D including both ER and industry (blue shades) and DP (red shades), for the estimated long-range budget needs taken from Figs. 5.1-4 and 5.1-5, respectively. The darker shaded portions of the bars indicate the construction costs of the ETF/LMF and NIF, respectively. The darker shaded portions of the bars indicate the construction costs of the ETF/LMF and NIF, respectively. A small fraction of the DP spending over the next decade, the ER spending is critical to insure the full completion of the ILSE program for heavy ion driver feasibility, the primary IFE driver candidate, by the time ignition in the NIF is achieved. This would allow a timely decision to proceed with the integrated ETF/LMF facility for IFE development following the NIF, leading ultimately to an IFE DEMO by 2025. The blue-shaded costs after 2015 include $\$ 600 \mathrm{M}$ for a DEMO upgrade, assumed to be provided by US industry and utilities.

ETF/LMF driver are also indicated in Fig. 5.1-6. In Figs. 5.1-4 and 5.1-5, construction costs for the NIF and the ETF/LMF include $\sim 20 \%$ contingency, but the uncertainty in the ETF/LMF driver is probably much higher. The entire integral cost of both IFE-specific and DP-IFErelevant costs are both probably low by another $10 \%$ for detailed items left out. Future WBS Level-two descriptions may reduce the higher percentage uncertainty in some items such as chamber and target systems for the high-pulse rate ETF/LMF (however, the fusion chambers are still not expected to cost as much as the driver, based on many IFE power plant studies). The most important perspective derived from Fig. 5.1-6 is that while the total R\&D contributions from ER and DP are both comparable over the next 30 years to the DEMO ( $\$ 4 B$ each), the total ER-IFE-specific costs over the next 10 years (largely for heavy ion driver development) is small compared to projected DPIFE-relevant spending ( $\angle \$ 400 \mathrm{M}$ for $\mathrm{ER}$ in contrast to $>\$ 2000 \mathrm{M}$ for DP to 2005). This is as it should be, since there is an urgency to implement SBSS 
in a timely manner to prepare for a Comprehensive Test Ban Treaty. While the ER spending on IFE is a small fraction of the DO spending over the next decade, the ER spending is critical to insure the full completion of the ILSE program for heavy ion driver feasibility, the primary IFE driver candidate, by the time ignition in the NIF is achieved. This would allow a timely decision to proceed with the integrated ETF/LMF facility for IFE development following the NIF, leading ultimately to an IFE DEMO by 2025.

\subsection{A Strategy for the Engineering Test Facility}

\subsubsection{Introduction}

If two features of the inertial fusion process were explored successfully, they can lead to significantly lower costs for demonstrating the feasibility of commercial electric power production from inertial fusion energy. First, fusion capsule ignition and burn physics are independent of reaction chamber size, and hydrodynamically equivalent capsules can be designed to perform at small yield, exactly as they do at large yield. This means that an engineering test of all power plant components and feasibility tests of reaction chamber concepts can be done at much smaller sizes (about 1-2-m first wall radius) and much lower average fusion powers (tens of MWs) than magnetic fusion development facilities such as the International Tokamak Experimental Reactor (ITER). The NIF, in producing similar fusion yields of $20 \mathrm{MJ}$, can therefore provide a target chamber environment (See Section 3) for testing the effects of materials on target emissions and on gas dynamics, which are relevant to an ETF. Second, the driver, which is the most expensive component of currently conceived IFE development facilities, can be used to support more than one experiment target chamber/reactor (simultaneously and/or sequentially). These two factors lead to lower development facility costs, modular facilities, and planning flexibility that will spread costs over time, or do several things in parallel thereby shortening the total time needed for development of Inertial Fusion Energy (IFE).

For one particular example assumed in Section 5.1 and 5.3 for costing purposes, a 5-MJ heavy ion ETF driver capable of both high pulserates for IFE tests, and high yield $(-400 \mathrm{MJ})$ ' for defense applications, is expected to cost only about $40 \%$ more than a $2-\mathrm{M}$ J driver producing much lower yields of $40 \mathrm{MJ}$ solely for reducedyield IFE testing. Thus, such a driver can support an added LMF mission ${ }^{22}$ by adding a separate target chamber dedicated to defense experiments, at an additional cost much less than the cost of a separate LMF facility with a 5-MJ driver. Thus, such a combined ETF/LMF facility would have a lower cost/benefit ratio for the combined DOE missions. The only reason to have separate LMF and ETF facilities would be, if the only driver options ready at the 2005 decision point (see IFE Development Strategy, Fig. 5.1-1) were capable only of single-shot operation, and if there were a pressing defense need to proceed expeditiously with an LMF.

Assuming adequate rep-rate drivers are available by 2005 , there would be other benefits to a driver capability for a combined ETF/LMF: the higher driver energy for the combined LMF/ETF mission would provide a larger driver energy margin to cover the uncertainties of focused ion beam spot size in initial ETF operation. Finally, once a suitable IFE fusion chamber technology was developed in the lowaverage power ETF test phase, a more capable ETF/LMF driver could then support a fewhundred MWe DEMO simply by adding a larger fusion chamber to operate at $200-300 \mathrm{MJ}$ yields and moderate pulse repetition rates of $2-3 \mathrm{~Hz}$. Thus additional cost savings are gained by allowing such an upgrade to a DEMO, and this facilitates US industries and utilities support of the DEMO upgrade.

In this section, we describe a fusion power technology development plan that takes aclvantage of the ability to test-chambers and reactor systems at small scale in order to reduce development time and costs. ${ }^{23}$ The additional economic benefits of using a single driver for a combined ETF/LMF are discussed in Sections 5.1 and 5.3. 


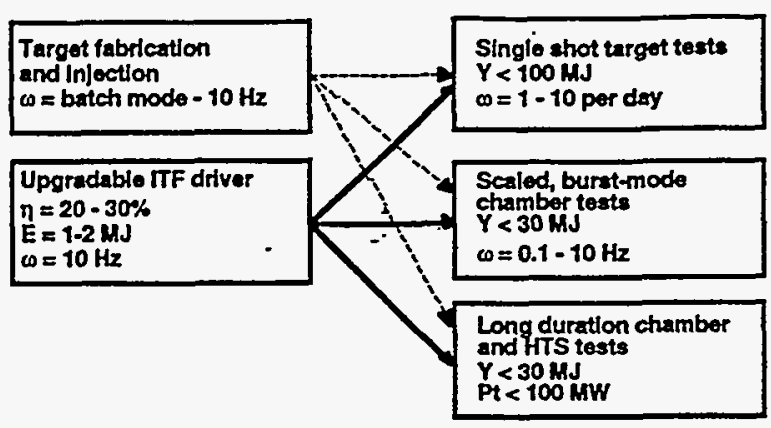

Figure 5.2-1. Schematic of the Engineering Test Facility. The ETF can be built in modules that can operate in parallel or series.

The ETF is discussed here in the context of a heavy ion driver for the reasons previously given. The principles of cost reduction (i.e., lowyield, lower power chamber testing) and rapid development (i.e., testing multiple chamber concepts with a single driver) are applicable to any driver which can operate multiple chamber via beam switching, movable chambers, or other switching concepts.

The Engineering Test Facility. The ETF, illustrated in Fig. 5.2-1, is a key component in a low-cost and rapid IFE development program. ${ }^{24}$ Multiple experimental chambers are envisioned, operating in parallel if the minimum time development program is desired. Initial experiments will demonstrate the first ignition with the ETF/LMF driver using a fixed-position, minimal-ignition, low-yield target. If the heavy ion driver is selected for the combined DP/ER mission, this would be the first ignition with a heavy ion driver. Following experiments will ignite a target "on the fly." Next, multiple-shot, low-yield experiments will be done in a burst mode to determine the pulse rate achievable in various reaction chamber concepts. Finally, longer duration experiments will demonstrate steady thermal power production and removal. With low-yield targets, even long-duration experiments can be done at estimated power levels of 50 to $100 \mathrm{MW}_{\mathrm{t}}$. Initially, targets for these burst-mode tests will be produced in batches. As the technology for continuous manufacture improves, and the need for longer duration tests grows, this technology will be incorporated into the experiments.
At this point, the ETF will have met all the objectives of a Pilot Plant: 25

1. Demonstrate proof-of-principle of a new technology;

2 Integrate, for the first time, all required systems at some scale;

3. Establish, at low cost and small scale, the technical feasibility of the concept; and

4. Demonstrate that the technological steps to a Demonstration Power Plant (DEMO) will primarily consist of scaling up from the Pilot Plant.

Demonstration Power Plant. In parallel with the ETF chamber experiments and prior to the DEMO, experiments will be done to improve target gain to that necessary for an $\eta G$ (driver efficiency-gain product) large enough to reduce the driver power requirement to an acceptably low value (typically $h \mathrm{~h}>10$ ). These experiments will be done in a separate, low-pulse rate (i.e., a single shot) chamber with a fixed target position but capable of higher yield. For the combined ETF/LMF, the LMF chamber serves this function.

If the required $\eta G$ is achieved, and if the long-duration, low-power chamber tests are successful, an integrated, larger scale fusion chamber could be added to the ETF/LMF driver. The power level and size of this fusion chamber would be chosen appropriate for the last demonstrations before the first commercial plant. At this time, the ETF would de facto become the DEMO without actually building a new separate large facility.

A DEMO addresses the issues of reliability, establishes scalability from a Pilot Plant, and is large enough to scale up to the first commercial plant (the Prototype Plant). The DEMO demonstrates licensibility, public acceptability, and cost viability. ${ }^{25}$ It is important that the DEMO-specific upgrade costs, and the DEMO operation be supported by an assumed consortium of US industries and utilities who would also be the commercial vendors and customers of the technology. .

\subsubsection{Scaling Chamber Designs}

A critical issue for the fusion power technology development path outlined above 
and the ability to keep the development costs as low as possible is how small the burst-mode test chambers can be made, and still accurately determine the pulse-rate capabilities of the various chamber concepts. In this subsection, we examined the phenomenology determining chamber pulse rate and how it scales with chamber size and target yield.

Fusion capsule ignition and burn physics are independent of reaction chamber size, and hydrodynamically equivalent capsules can be designed to perform at small yield, exactly as they do at large yield. This means that an Engineering Test of all power plant components and feasibility tests of various reaction chamber concepts can be done at much smaller sizes (about 1-2-m first wall radius) and much lower powers (tens of MWs) than magnetic fusion development facilities such as ITER. In this Section we discuss some important considerations when scaling chambers to do simulations at low yield.

\section{$X$-ray and Debris Effects in Scaled}

Chambers. Some of the most important effects in an IFE chamber are related to the intense energy deposition by $x$ rays and target debris. The sudden vaporization of a thin (10s of $\mu \mathrm{m}$ ) layer of the first surface causes an impulsive pressure load on the underlying material. The vaporized material fills the center of the chamber with a hot plasma that reradiates and vaporizes more material. As this vapor cools, it condenses, and the vapor density in the chamber returns to a low enough level that allows the beams to propagate through the chamber for the next pulse. Understanding and being able to predict the chamber dynamics are the key to determining the maximum possible rep rate for the chamber design, which can affect the overall plant economics.

A series of CONRAD ${ }^{26}$ calculations was carried out to examine key response functions for both full-size chambers (i.e., for future commercial power plants) and small chambers (characteristic of what we envision for the ETF). These calculations were conducted for three representative chamber types: HYLIFE-II, 27 which features a thick liquid (Flibe) wall blanket; Osiris, ${ }^{28}$ which has a Flibe-filled, porous-fabric blanket; and Cascade, ${ }^{29}$ which has a flowing granular blanket (the innermost layer is carbon). The inner radius of each ETF blanket was set so that the total $x$-ray and debris fluence per shot $\left.0 / \mathrm{cm}^{2}\right)$ was the same as for the corresponding full-size design.

A summary of some key results is given in Table 5.2-1. In all cases, the thickness of material vaporized by $x$ rays, debris, and reradiated energy is about the same in the ETF and full-size chambers. Peak pressures and impulsive loads are also very close. Although not shown here, the time dependent features of plasma temperature, radiation temperature, etc., demonstrate similar characteristics.

The final parameter listed in Table 5.2-1 is the vapor energy per unit volume within the first wall at the end of the calculation ( $6 \mu$ s in these cases). This parameter is related to the pressure in

Table 5.2-1. Results of CONRAD chamber dynamics calculations.

\begin{tabular}{lcccccc}
\hline & \multicolumn{2}{c}{ Cascade } & \multicolumn{2}{c}{ HYLIFE-II } & \multicolumn{2}{c}{ Osiris } \\
\hline \hline Scale & Reactor & ETF & Reactor & ETF & Reactor & ETF \\
Yield $(\mathrm{MJ})$ & 375 & 31.5 & 350 & 31.5 & 430 & 31.5 \\
First surface radius $(\mathrm{cm})$ & 310 & 86.2 & 50 & 14.4 & 350 & 90.9 \\
X ray and debris $\left(\mathrm{J} / \mathrm{cm}^{2}\right)$ & 93 & 93 & 3340 & 3340 & 56 & 56 \\
Ablated thickness $(\mu \mathrm{m})$ & 2.9 & 3.1 & 200 & 190 & 20 & 18 \\
Peak pressure $(\mathrm{GPa})$ & 17 & 17 & 90 & 200 & 30 & 28 \\
Impulsive pressure $(\mathrm{Pa}-\mathrm{s})$ & 32 & 31 & 1900 & 1700 & 120 & 100 \\
Reradiation to surface $\left(\mathrm{J} / \mathrm{cm}^{2}\right)$ & 2.4 & 0.6 & 1100 & 1300 & 9.5 & 4.8 \\
Cavity E/V $\left(\mathrm{J} / \mathrm{cm}^{3}\right)$ & 0.6 & 2.5 & 120 & 390 & 0.5 & 2.0 \\
\hline
\end{tabular}


the vapor and is important for the long-term mechanical response of the blanket and chamber structures to the pressure pulse. It will also affect the rate at which vapor blows through the HYLIFE-II jets and the rate at which hot Flibe vapor will be driven into the condensing pool at the bottom of the Osiris chamber.

The $\mathrm{J} / \mathrm{cm}^{3}$ scales approximately as $\mathrm{Y}^{-1 / 2}$ since the energy in the vapor is proportional to the yield, while the volume scales as $\mathrm{R}^{3}$, which in turn is proportional to $Y^{3 / 2}$ when the first surface $x$-ray and debris fluence constant is held constant. For a $\sim 30-\mathrm{MJ}$ ETF, the yield is down by a factor of 11-14 (for chamber designs we considered) and the $E / V$ is about a factor of 3-4 higher in the small chambers. Conducting lower yield experiments in the ETF chamber (by reducing the driver energy or modifying the target design) would allow the E/V to be matched, but at a first-wall fluence that is less than for a full-size chamber. A series of experiments having a range of yields should provide enough information to allow designers to scale the results to the DEMO size chamber.

Another factor to be considered in the chamber response to $x$ rays and debris is that at very low target gain, the driver energy increases the fraction of the yield that is in $x$ rays and debris. At high gains, the contribution of the beam energy is minimal, but at low gain the contribution is significant. For example, if the capsule $x$-ray and debris fraction is fixed at $24 \%$, the total $x$-ray and debris fraction varies from $25 \%$ at $G=100$ to $37 \%$ at $G=15$. This will be important for designs where the combination of neutrons and short range energy effects are both important; HYLIFE-II is an example.

Neutron Effects in Scaled Chambers. We have also examined how neutron energy deposition profiles change as a function of yield. Again, the inner radii of the blankets were scaled as $Y^{1 / 2}$. Results are shown for HYLIFE- $I I$ in Fig. 5.2-2. The exponential fall-off through the blankets is similar at all yields. The specific energy deposition $(\mathrm{J} / \mathrm{g}$ ), however, decreases with decreasing yield, and the fall-off is greatest for the innermost zones of the blanket. This is basically a geometric effect. To first order, a fixed fraction of the neutron energy will be deposited

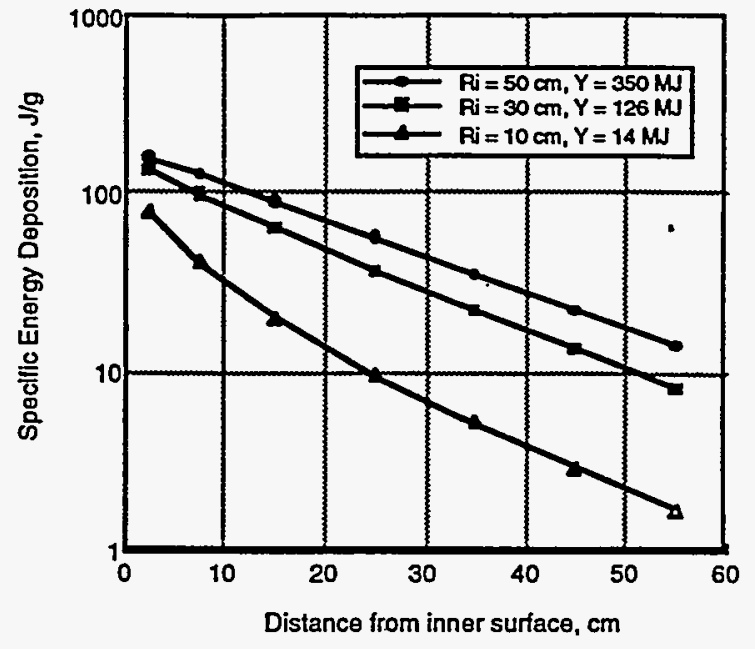

Figure 5.2-2. Neutron energy deposition profiles in HYLIFE-II blankets for different yields.

in a given thickness $(\Delta R)$ of the blanket. Therefore, as the yield approaches zero, the energy deposited in a fixed thickness approaches zero, while the volume of that blanket region approaches the volume of a sphere of thickness $\Delta \mathrm{R}$. The effect is most pronounced in designs that have a small inner radius for the fullsize chamber.

While the $\mathrm{J} / \mathrm{g}$ in the innermost zone of a lowyield HYLIFE-II ETF chamber may be a factor of two lower than for the full-size chamber, it is still higher than the $10 \mathrm{~J} / \mathrm{g}$ needed to break up the Flibe jets. Therefore, the gross effects of jet breakup will still be observed at yields of 10s of MJ.

\subsubsection{Scaling Heat Transfer Components}

As previously discussed, the ETF will also conduct long duration tests to examine the heat transfer and transport characteristics of the designs. These tests must be conducted at a power level that allows scale-up to DEMO-size heat transfer system (HTS) components. Assume, for example, that the commercial plant has a thermal power of $2800 \mathrm{MWt}$ and has four parallel heat transfer loops supplying steam to the turbine. The DEMO will only have to demonstrate a single loop and only at about $1 / 3$ scale in power. Therefore, the DEMO will operate 
at a power of $\sim 230 \mathrm{MWt}$. The DEMO chamber will only handle $1 / 12$ the power of a commercial chamber. This reduced power could be obtained with somewhat lower yield targets and lower rep-rate so that each of these parameters is closer than $1 / 12$ to the full-size plant parameters. For example, the full-scale Osiris operates at $430 \mathrm{MJ}$ and $4.6 \mathrm{~Hz}$. An Osiris DEMO could operate at 150-200 MJ and $1.5-1 \mathrm{~Hz}$ for the power test. It would still be possible to do burst-mode tests at the full $4.6 \mathrm{~Hz}$, but it would not be necessary to have HTS components capable of handling the higher power.

The ETF would also only test a single heat transfer loop, but at a 1/3 scale of the DEMO. For this particular example, the ETF power level would be about $75 \mathrm{MWt}$. With yields of 20 to $30 \mathrm{MJ}$, this would require rep-rates of $\sim 3 \mathrm{~Hz}$. Again, burst-mode experiments at higher reprates would already have demonstrated the reprated capabilities of the designs.

\subsubsection{Conclusions}

In addition to sharing a single driver in the ETF/LMF, one of the key factors in minimizing development costs is the ability to rapidly test multiple chamber concepts at low yield and power. We find that critical chamber phenomenology (e.g., vaporization and condensation dynamics) can be tested with lowyield targets using very compact chamber designs. A single ETF can test several different chamber concepts, a possibility that is unique to IFE. Less than $100 \mathrm{MWt}$ will be required to test heat transfer equipment. This power level is based on reasonable assumptions about component capacity scale-up between each successive facilities; the power is not determined by the ignition physics as in MFE.

\subsection{A Common Ion Driver for Both Defense and Energy Applications}

\subsubsection{Background-Present Strategy}

The presently stated goal of the National ICF Five-Year Program Plan ${ }^{30}$ is "to produce small thermonuclear explosions in the laboratory, with fusion yields of 200 to $1000 \mathrm{MJ}, . .$. for defense and civilian (energy) applications ..." For this laboratory microfusion facility, DOE's Defense Program is developing three candidate drivers: glass lasers, $\mathrm{KrF}$ gas lasers, and light ion pulsed power accelerators-with acknowledgment that DOE's Energy Research Program is developing heavy ion accelerators for an IFE engineering test facility, which is a fourth option for an LMF driver.

Figure 5.3-1 displays DP's present strategy from the above report, which is also reflected in the 1992 National Academy of Sciences (NAS), ${ }^{31}$ Fusion Power Advisory Committee, 32 and National Energy Strategy reports, ${ }^{33}$ all of which call for both an LMF and an ETF starting after laboratory ignition is first demonstrated in the NIF. In parallel with the NIF, both DP and ER would be developing drivers capable of greater energy than the NIF 1.8-MJ glass laser, in order to produce higher yields for extended weapons physics and nuclear weapons effects simulation capability for LMF, and for higher gain for ETF, respectively. Decisions of which driver to use for the LMF and the ETF would be made at the time ignition is expected to be demonstrated in the NIF, approximately 2005 . The main difference in requirements for the $\mathrm{LMF}$ and $\mathrm{ETF}$ drivers is that an LMF driver can be single shot, while an ETF driver must have rep-rate capability, efficiency, and lifetime for a much greater number of shots. For the latter facility, NAS and FPAC recommended development emphasis on heavy ions, due to the need for reliable, efficient, and high rep-rate operation for IFE development. 


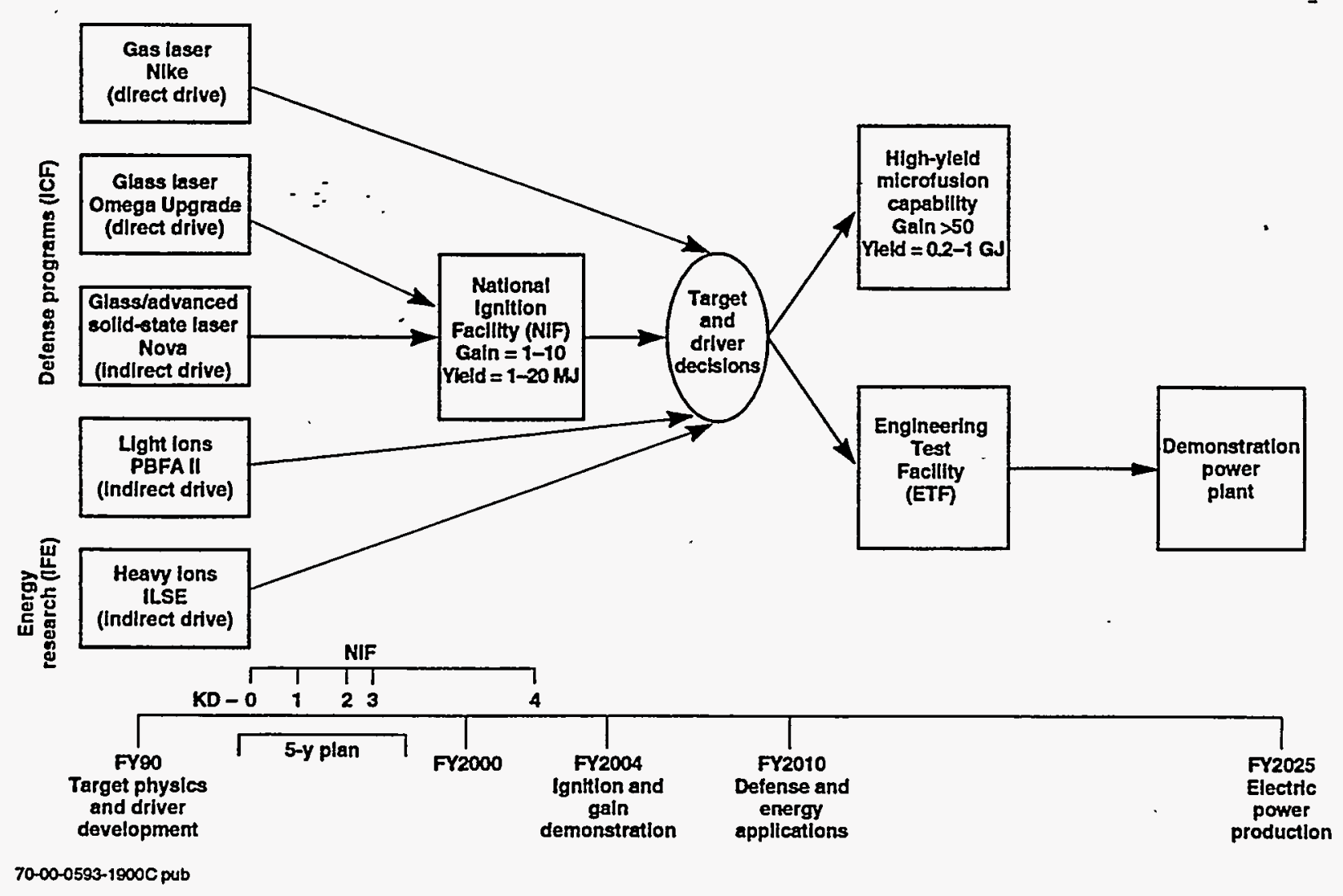

Figure 5.3-1. Defense Program's present strategy for ICF.

\subsubsection{A Proposed New Strategy}

Predicated on the assumption that a near optimum ion driver and target can be found, Fig. 5.3-2 depicts a new strategy for a combined LMF/ETF facility sharing a common ion driver, with a common ion driver development program supported by both DP and ER. This combined facility should be capable of both the LMF and ETF missions, most likely in separate target chambers optimized for each mission. The LMF mission is to provide sufficient ICF yield to extend weapons physics and NWES capabilities beyond those of the NIF. The ETF mission is to develop IFE fusion chamber technology and driver performance and reliability at high reprates representative of an IFE power plant. Most system studies for IFE power plants find an optimum yield of a few hundred megajoules, which matches the desired LMF yield for defense program applications. The goals of this new strategy are:
1. Increase total resources for ion driver technology for both LMF and ETF, through cooperation on elements leading to a generic ion driver.

2 Reduce the total cost of accomplishing the LMF and ETF missions through a combined facility sharing a common driver.

3. Increase motivation for the next inertial fusion facility after NIF by offering more diversity for its use and benefits.

The prime motivation for this new strategy is to make the next step after NIF, both more affordable and desirable, and to increase the likelihood of having a driver technology ready to support a decision for that new facility by the time ignition is achieved on the NIF.

The multiuser strategy also motivates an ion driver architecture that allows switching the ion beams between several different target chambers, as depicted in Fig. 5.3-3, or alternatively, by sequentially moving the target chambers, as depicted in Fig. 5.3-4. Weapon physics 


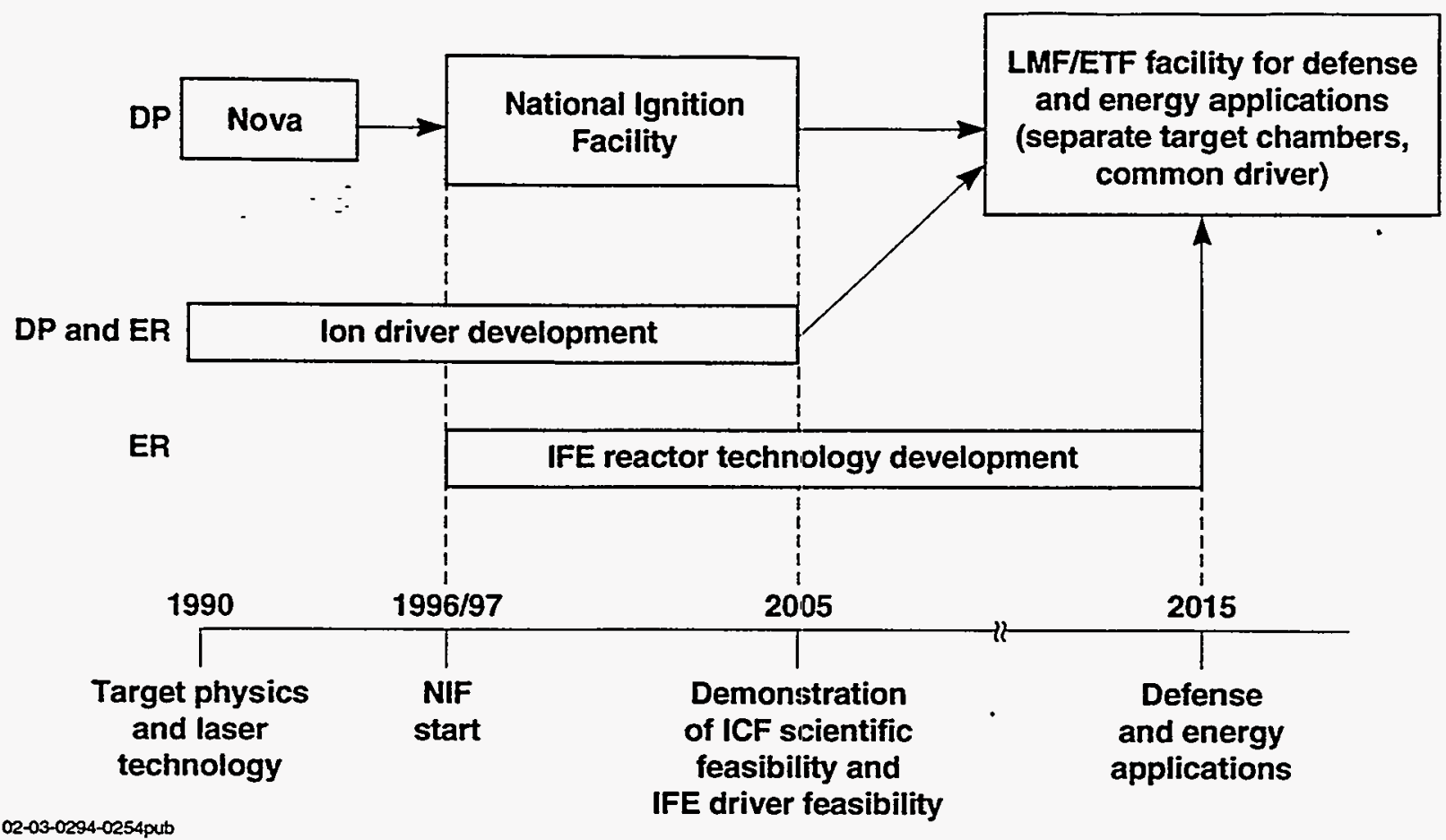

Figure 5.3-2. A new strategy for a combined LMF/ETF facility sharing a common ion driver.

experiments and NWES tests will require extensive and varied diagnostic access in an LMF chamber, with probably many different types of specialized targets that would be fired only in single shots, or for a very limited number of shots. Target chambers for defense experiments may also have to accommodate special ES\&H issues. The types of target chambers for IFE development needs, on the other hand, are likely to be quite different from that of an LMF, in order to qualify blankets for the DEMO. Demonstration of blanket candidates for tritium breeding selfsufficiency is a key role of the ETF; and integrated blanket tests, as opposed to small blanket test modules, may be required. The Integrated Test Facility (ITF), a WJSA/LLNL study of a low-cost version of an ETF, ${ }^{34}$ shows that several reduced-scale target chambers would be needed to test various candidate tritium breeding blankets and liquid-wall or protectedwall schemes to select a chamber concept for an IFE DEMO. Still other types of target chambers are likely implied for other potential users, such as for materials testing as depicted in Fig. 5.3-3 and 5.3-4.

Figures 5.3-3 and 5.3-4 illustrate another highly desirable feature for a suitable ion driver architecture for the LMF/ETF: an ability to build such a driver capability efficiently in two or three upgradable stages, by either adding accelerator sections to increase voltage (Fig. 5.3-3) or by aclding accelerator modules to increase beam current (Fig. 5.3-4). This efficient upgradability feature can allow a large driver to be built in stages to reduce risk, while avoiding long delays implied by separate facilities for each step. Development of one additional new facility on a site suitable to go all the way to DEMO in a series of upgrades, is probably the only way an IFE DEMO could be built by the 2025 schedule envisioned by the 1992 National Energy Policy Act. Ion driver research in both DP and ER should have a goal of determining an optimum accelerator architecture, ion mass and current per beam by 2000 to have a common driver program in place by the time ignition is achieved in 2005. 


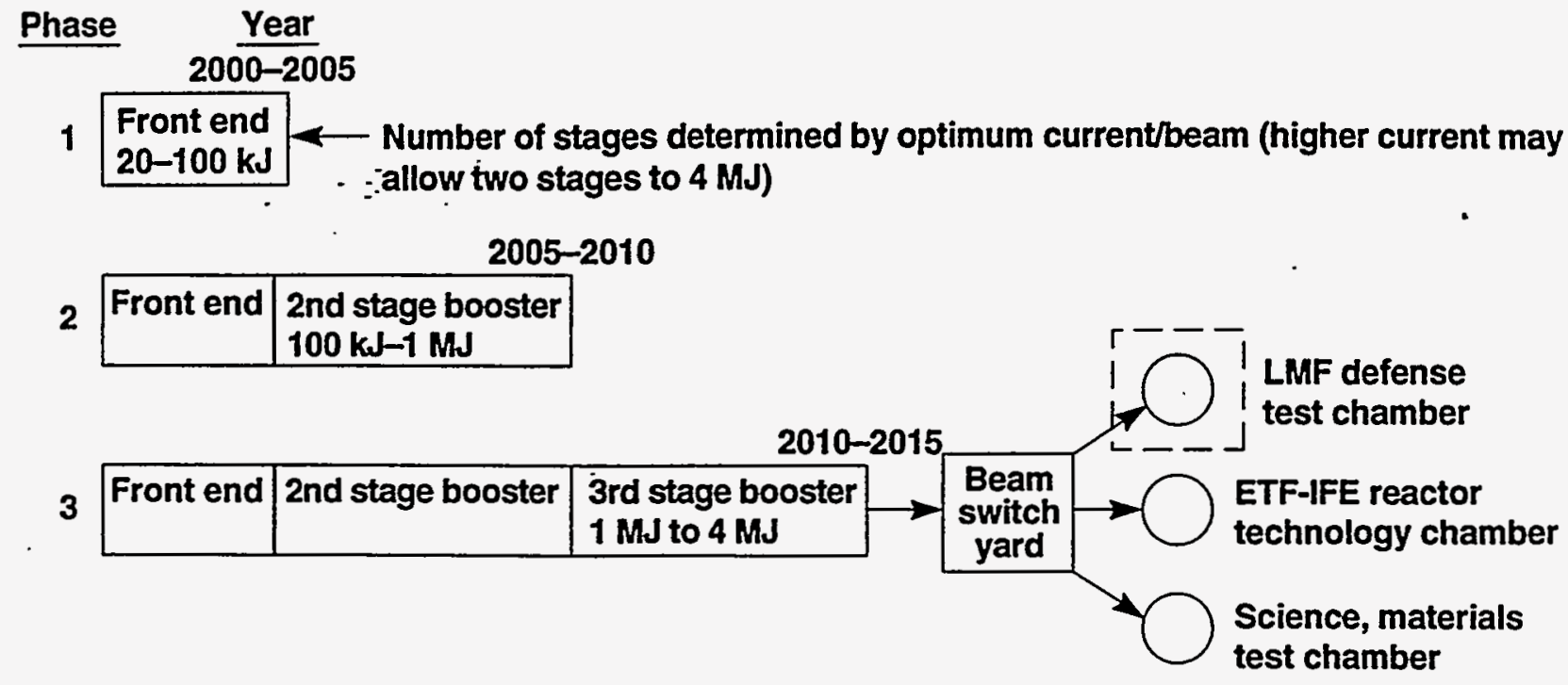

4 Upgrade to DEMO (increase driver availability, new reactor chamber, net power) $02-03-0294-0256$ pub

Figure 5.3-3. An ion driver architecture that allows switching the ion beams between several different target chambers.

1 module

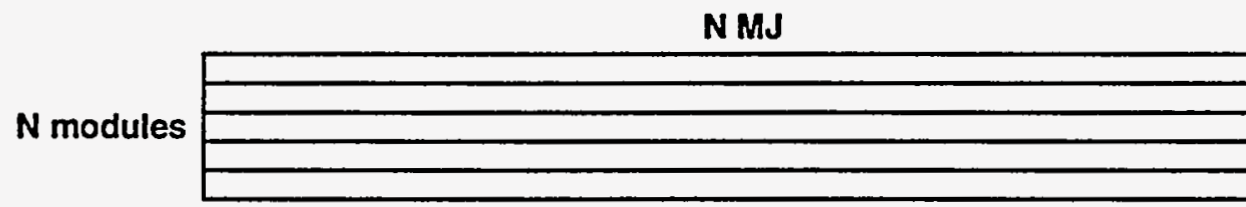

02-03-0294-179 pub

\section{$\sim 1 \mathrm{MJ}$}

Movable chambers

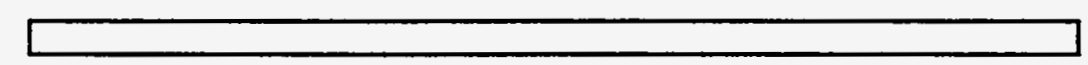

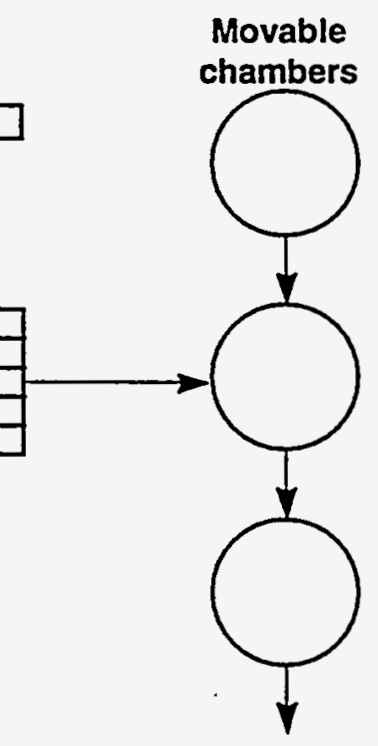

Figure 5.3-4. A facility architecture with sequentially moving the target chambers. 
Then, to test the chosen LMF/ETF driver at reduced scale without committing the full cost of the LMF/ETF, an intermediate accelerator prototype should serve as the first stage of an upgradable LMF/ETF-(Fig. 5.3-3), or as the first several modules (Fig. 5.3-4). The number of stages or modules for each upgrade is an optimization that can be decided later as the optimum current per beam is better determined, before the sequence depicted in Fig. 5.3-3 or 5.3-4 would begin.

\subsection{The Contributions of ICF Core Science and Technologies to IFE}

\subsubsection{Introduction}

The target chamber core technology development program focuses on key development issues to be resolved in the NIF design phase. ${ }^{1}$ The natural progression of this effort leads into issues related to Inertial Fusion Energy. By developing key technologies such as predictive capabilities, material response validation, and decontamination, a solid scientific foundation is laid for NIF and all future advanced ICF facilities. The proposed development activities for NIF that have the most applicability to IFE include:

- Characterization of target emissions.

- Development of first wall and target positioner protective coatings.

- Development of electrical diagnostic component vulnerability and hardening techniques.

- Development of target emplacement, positioning and injection techniques.

These are briefly discussed in the following subsections.

Some of the other NIF technology development activities may have indirect benefits to future IFE systems, but the technologies themselves are not releyant to high pulse rep-rate IFE power plants. These other areas are:

- Development of an absorber for unconverted laser light.
- Development of a disposable, one-shot final optic.

- Development of an automated tritium and activated debris decontamination system $\left(\mathrm{CO}_{2}\right.$ cleaner $)$.

These are discussed in Ref. 35

\subsubsection{Characterization of Target Emissions}

A quantitative understanding of the source terms for and effects of $x$ rays, $\gamma$ rays, neutrons and debris is key to the design of the target area. These source terms and their effects on the target chamber significantly influence the target area design and ultimately the success of the facility. The maximum combined $x$-ray and debris yields could be as high as $5.8 \mathrm{M}$ ] for the $5-\mathrm{m}$ radius NIF chamber compared to $50-\mathrm{kJ}$ yield for the $2.2 \mathrm{-m}$ radius Nova chamber. This factor of $\sim 20$ higher energy fluence at the first wall suggests that $x$-ray ablation of the first wall, deposition of debris and ablated material on optics, and activation of entrant diagnostics, can be major obstacles to the successful operation and maintenance of the NIF facility.

The technical objective of the target emission characterization task is to develop the ability to predict spectra and fluence of $x$ rays, gamma rays, neutrons, shrapnel and debris to within $30 \%$ using unclassified computer codes. Not only must the fluence, spectra, and pulse length be understood but the directionality and orientation to the target and chamber are critical factors as well. Results and predictions from this area drive the baseline target chamber design. Goals for the target emission characterization tasks are (for each type of emission, $x$ ray, debris, shrapnel):

- Develop unclassified predictive capabilities.

- Develop methods for diagnosing emissions.

- Predict Nova emissions and confirm experimentally.

- Predict NIF emissions and confirm experimentally on Nova.

- Confirm NIF emissions on NIF.

- Predict LMF-ETF and IFE emissions and confirm on NIF. 
- Publish Periodic Target Emission Reports.

To prepare the NIF conceptual design, the capabilities of several emission prediction codes were surveyed. The capabilities and limitations of each code are briefly described in the following paragraphs.

HYADES ${ }^{36}$ is a radiation/hydrodynamics code in 1-D (planar, cylindrical or spherical). This code is well-suited to high-intensity laser and $x$-ray interactions with materials, where the lowenergy material properties (such as strength) are not significant. Thermonuclear burn can be modeled. Features under development include ion deposition, material strength models, and 2-D capabilities.

CONRAD ${ }^{37}$ is also a radiation/ hydrodynamics code in 1-D (planar, cylindrical or spherical). This code was written to model target chamber environments, so both x-ray and debris (ion) energy loadings are implemented. Additionally, thermal radiation from hot vapor/ions can deposit energy on surfaces. The ablation model includes the effects of thermal conduction. Features under development include laser deposition and 2-D capabilities.

TSUNAMI $^{38}$ is gas dynamics code in 2-D (planar or cylindrical). This code uses a simple ablation model to determine the initial conditions for the motions of ablated vapor. A target can be included by specifying an initial mass and energy of a few cells. Real gas equations of state can be included, as these are important for dissociation and ionization of species. Features under development include radiation transport and condensation boundary conditions.

PUFF2D ${ }^{39}$ is a shock/hydrodynamics code in 2-D. This code is used to model the effects of $x$ ray-generated (and other) shocks on condensed phases of materials. It includes extensive material and fracture models to predict failure. The initial conditions (energy deposition profile) must be determined with a separate code, so integration with such a code perhaps HYADES is under consideration.

DYNA2 $\mathrm{D}^{40}$ is a shock/hydrodynamics code in 2-D. A 3-D version is also available (DYNA3D). This code is used to model the effects of shocks and other transient loads on solid materials. It includes numerous material and fracture models. The initial conditions (energy deposition profile) for chamber modeling must be determined with a separate code.

All of these codes have been successfully used in applications both within the LLNL ICF program and outside of it. However, to date, none of these codes have been validated to the energy deposition levels anticipated for ICF missions. The accuracy with which these codes can calculate target emissions and its interaction with the target chamber must be evaluated as part of this core technology task. Developing these predictive capabilities will be essential for moving to the ETF phase of IFE, where it is anticipated that several first-wall and blanket concepts will be experimentally evaluated.

\subsubsection{First Wall and Target Positioner Protective Coatings}

The development of first wall and target positioner protective coatings is a primary part of the target chamber core technology program. The technical objective of the first wall development task is to prevent degradation of the target chamber and final optic by material ablated by $x$ rays, and shrapnel generated by the NIF target. While IFE chambers do not use coated metal walls, the predictive capabilities developed as part of this activity will be useful to first-wall design issues for IFE.

This development task entails a cost benefit study of the effects of the various target emission source terms on a number of candidate materials. Code calculation and experiments on Nova will be used to determine the various effects on the candidate wall materials. The net fluxes that will impact the debris shields will also be assessed. This work will lead to the selection of an optimum first-wall material. Benchmarking of code calculations with experimental results on Nova will lead to validated predictive capability for NIF and LMF/ETF first-wall materials. Goals for the first-wall materials task are:

- Establish bounds on target emission source terms.

- Expand the list of potential first wall materials.

- Develop coating application technology. 
- Investigate the effects of source terms on each candidate first-wall material, including repetitive pulses and decontamination.

- Develop and use computer codes to study these effects.

- Perform material tests on Nova and benchmark codes.

- Use benchmark codes to predict the optimum material for NIF and LMF/ETF.

Experiments to test various candidate firstwall materials will be performed on NOVA and Saturn, and on NIF as it comes on line. Comparison with code predictions will lead to benchmarking of the codes. The codes can then Pbe used to make predictions for the higher yield NIF shots and eventually for LMF/ETF parameters.

\subsubsection{Diagnostic Component Vulnerability and Hardening Techniques}

The conceptual design of the NIF target area instrumentation system demands that the instruments must survive in a hostile $x$-ray, neutron, gamma ray, EMP, and debris environment. In addition, data integrity must be maintained for high-resolution images and subnanosecond transient signals while maintaining a demanding operational shot schedule. The technical objective of this task is to develop hardening techniques to allow the required diagnostics instrumentation to survive target area emission and record data.

As IFE develops to a commercial power source, the number of in-chamber diagnostics will likely decrease (e.g., target implosion diagnostics will be eliminated since targets will have been thoroughly developed and tested; they must operate reliably at high rep-rate for IFE to be an acceptable energy technology). Other types of in-chamber diagnostics, however, will be needed (e.g., to measure flow rates, vapor conditions, etc.), particularly in the ETF and DEMO phases of a development program. Therefore, the lessons learned in hardening diagnostics for the NIF will be beneficial to the development of IFE.
The goals of the electrical diagnostic component vulnerability and hardening techniques plan are:

- Determine the neutron, gamma, $x$-ray, EMP, and debris environments expected for the NIF diagnostics.

- Determine the susceptibility of each type of diagnostic component.

- Determine the vulnerability of each diagnostic.

- Develop hardened instrumentation to meet the requirements of the NIF environment.

\subsubsection{Target Emplacement, Positioning and Injection Techniques}

The target emplacement, positioning, and injection systems provide for transport of a room temperature or cryogenic target from the target preparation facility outside the NIF target chamber to the center of the chamber, and then position the target in alignment with the focal spot of the laser beams. The system must then hold the target in position until the laser is fired, maintaining the environment of the target. For a cryogenic target, systems must be developed to layer, characterize, inspect, and transport the target within the facility.

For future IFE power plants, an injection system must be developed which can deliver a target several times per second. It is anticipated that these targets will be cryogenic and will have very stringent positioning requirements. The target injection system must launch targets at high velocity $(\sim 150 \mathrm{~m} / \mathrm{s})$ with excellent repeatability in terms of positioning and orientation. Methods for accomplishing this from a large distance, $\sim 10 \mathrm{~m}$, must be developed for these concepts to move forward. It has been proposed to test an injection system on the NIF.

The specific development goals of this activity are to:

- Determine the vibrational characteristics of the baseline target inserter.

- Determine the vibrational tolerance of the baseline target during transport. 
- Develop and test an inserter concept which meets the vibrational and positioning requirements.

- Develop a cryogenic target inserter.

- Develop a preconceptual design of a rep ratable target inserter for the IFE program.

The major interfaces for this development activities are the Target Alignment System development, the Target Fabrication and Development Activity, and the National Cryogenic Target R\&D Program. Both of the target programs are outside the NIF project, but it is absolutely necessary that a good working relationship be established with these activities.

\subsection{Role of the NIF in IFE Laser Driver Development}

\subsubsection{Overview}

The preceding chapters pertain to an IFE power plant using a heavy ion driver, which is the most promising candidate for an IFE driver because of its anticipated high efficiency $(\geq 25 \%)$. Many of the experiments proposed above for the NIF would also benefit the development of a laser driver, which is an alternative to a heavy ion driver. In fact, the operation of the NIF will itself assist the development of any laser driver in terms of broad considerations such as the following:

1. Deployment of hundreds of beams that must be aligned, synchronized, balanced for uniform delivery of energy, and transported through a switchyard to interface with a target chamber.

2. Arrangement of the beams into cones or other patterns subtending large solid angles upon entrance through the envelope of the target chamber (or small solid angles, if target gain permits).

3. Transport of UV light, which requires the development of high-damage threshold optics and coatings.
4. Use of the modularity of the laser system in the formation of a suitable pulse shape for a high-gain target.

5. Development of bandwidth (i.e., a band of wavelengths) and the investigation of the effect of bandwidth on target performance. Although applicable in such broad ways to the development of any laser driver for IFE, the NIF can play an additional, special role for a particular type of laser driver: a diode-pumped solid-state laser.

A DPSSL is a promising alternative to a heavy ion driver for IFE, that shares many features in common with the NIF laser. ${ }^{41}$ One major difference is that the NIF laser uses Nd:glass side-pumped with flashlamps, while a DPSSL uses a special crystal, $\mathrm{Yb}^{3+}$-doped $\mathrm{Sr}_{5}\left(\mathrm{PO}_{4}\right)_{3} \mathrm{~F}$ (called $\mathrm{Yb}: \mathrm{S}-\mathrm{FAP}$ ), end-pumped with laser diodes. This difference, however, is overshadowed by the similarity of the designs in almost every other respect. Both use four-pass regenerative amplifiers operating near $1.05-\mu \mathrm{m}$ wavelength with an optical switch composed of a Pockels cell and a polarizer. Both use similar beam transport equipment and both use harmonic conversion to $3 \omega$ in $\mathrm{KD}^{*} \mathrm{P}$ crystals. Both must interface with high-gain targets using hundreds of laser beams configured around a target chamber. In addition, the DPSSL output is only about twice as great as that of the NIF laser. 41

The DPSSL is a more attractive option for IFE than other laser driver candidates because it is highly modular, and it can be functionally tested at small-aperture scale. This feature, together with the reliance of the DPSSL design on the rather mature experimental foundation based on ICF research using solid-state lasers such as Nova, enables the DPSSL development costs to be rather low. Operation of the NIF itself will, therefore, serve to validate much of the basic operation of a DPSSL as a promising laser option for an IFE driver. In this sense, the NIF is the next logical step in the direct development of a DPSSL driver for IFE. 


\subsubsection{Normal NIF Operation Verifies DPSSL Functionality}

Because many features of the NIF system are related to those proposed for the DPSSL driver for $\mathrm{IFE},{ }^{41}$ the integration, startup, and operation of the NIF laser will demonstrate the functionality of many aspects of the DPSSL driver. We emphasize this verification of DPSSL performance and equipment functionality in this section. No additional experimental operations or equipment are required; normal NIF operation will suffice.

The goals for the use of the NIF in verifying the functionality of a DPSSL driver are the following:

1. To demonstrate the functionality of the NIF laser equipment in common with a DPSSL driver. This includes, for example, the functional testing of the following equipment:

- Front-end electronics (optical pulsegeneration system). Issues include the type of design, the role of optical fibers, and the way to generate bandwidth.

- Four-pass regenerative laser amplifier. This includes an optical switch composed of a large-aperture Pockels cell and a polarizer. Issues include pulse injection, spatial-filter and pinhole operation, optical relaying, avoidance of ghost foci, technique for beam switchout, and method of apodization.

- Harmonic converters for efficient conversion from $1 \omega$ to $3 \omega$.

- Computerized control equipment for aligning individual beams, and synchronizing and energy-balancing many beams.

- Mechanical and optical equipment to interface hundreds of laser beams to the target chamber.

2 To investigate and understand issues that might impair laser operation for the NIF in the same way that they might for a DPSSL, or that relate to the efficiency of the NIF laser in the same way as for a DPSSL. This includes, for example, the understanding of the following phenomena:
- Damage thresholds for optics and coatings. This includes measuring thresholds, and developing materials and coatings with higher optical (and $x$-ray) thresholds.

- Pulse shaping. The issue is what method to use to produce a particular output pulse shape (e.g., front-end electronics, or a configuration of beams to accomplish optical addition of beams spaced in time).

- Aligning and operating laser beams to achieve adequate symmetry, synchrony, and energy balance.

- Beam transport. Issues include whether beam tubes should be used (or just air transport) and where the beams should be converted to $3 \omega$.

- Survivability of the final optics against $x$ rays, target-plasma debris, neutrons, and condensates.

- Focusability of $3 \omega$ beams on target. This includes understanding the effect of beam quality on the losses in getting the laser energy into a hohlraum.

3. To test laser-driven targets and to understand the workings of target hohlraums at the NIF scale. This includes the following research conducted on the NIF that is directly applicable to the operation of a DPSSL-driven IFE plant:

- Losses in getting $3 \omega$ beams into a hohlraum. This includes effects caused by the closure of the laser entrance holes, and by stimulated Raman scattering of the beams in the plasma created in the chamber gas near the target. (The chamber gas near the target can be ionized by a) multi-photon ionization, b) $x$ rays emerging from the hohlraum, or c) knock-on electrons.)

- Laser-plasma instabilities in a hohlraum.

- Symmetrization of beam energy on hohlraum walls.

- Efficiency of conversion of laser light to $x$ rays.

- Symmetrization of $x$ rays in the hohlraum

- Absorption of $x$ rays by a capsule. 
- Capsule implosion, fuel ignition, and fusion bum propagation.

- Disassembly of a hohlraum after ignition.

\subsubsection{Technical Background and IFE Development Activities on the NIF}

The normal operation of the NIF will assist in the development of DPSSL components in many ways. The design of the NIF laser front end, for example, will provide information that will aid in the selection of the type of system most appropriate for the DPSSL, especially in connection with the role of optical fibers, the way to generate bandwidth, and the role played by the front end in the formation of the desired output laser pulse shape. Although the electronics used for a DPSSL-driven IFE plant may be different because of technological improvements over the next few decades, the functionality of the two systems is similar. Both must produce a pulse for injection into the main amplifier, and the pulse must be suitably shaped and preamplified to maximize the extraction efficiency of the gain medium in the main amplifier.

The configurations of the amplifier cavities for the NIF laser and a DPSSL are different because the NIF laser uses Nd:glass side-pumped by flashlamps, while a DPSSL uses Yb:S-FAP end-pumped by laser diodes through the cavity mirrors. The DPSSL must operate at about $10 \mathrm{~Hz}$, so the gain medium and other optical elements must be gas-cooled. ${ }^{41}$ Nevertheless, the basic operation of the two cavities bears many similarities. Both must address optical relaying, spatial filtering without damage to the pinholes, apodization, configuration of internal beam dumps, and method of beam switchout. Current designs for the NIF laser and a DPSSL both rely on a large-aperture Pockels cell and a polarizer for switchout. Both the NIF laser and a DPSSL must be concerned about phenomena that affect extraction efficiency, such as B-integral, first-tolast-photon gain ratio, and optical damage thresholds (which relate to the development of more robust optical materials and coatings).

The DPSSL and NIF systems use similar beam transport, and both envision the use of
$\mathrm{KD}^{*} \mathrm{P}$ crystals for harmonic conversion to $3 \omega$ (although the DPSSL converter must be gascooled). These systems must rely on computerized alignment equipment and software to align hundreds of laser beams. A switchyard is required for both facilities to redirect the beams and position them appropriately around the target chamber. Both facilities must be concerned about the optimum location of the harmonic conversion (considering how far and through which components it is desirable to transport $3 \omega$ instead of $1 \omega$ ), and both must address the survivability of the final optics. Because the NIF debris shields may have to be changed after every high-yield shot, facility personnel will naturally seek ways to protect the final optics from $x$ rays, plasma debris, particulates, and condensates; such measures may be highly relevant for the protective methods eventually deployed for a DPSSL. The efficiency of any laser driver delivering beams into a hohlraum depends on the focusability as determined by the beam quality, which depends on the wave-front distortions that are introduced and that accumulate throughout the laser because of imperfections in the optical elements. The investigation of these effects for the NIF will be directly applicable to a DPSSL, even though the DPSSL beams may be subdivided into smaller subapertures. Experiments on the NIF to investigate stimulated Raman scattering of the laser light near the target, especially at higher target-chamber pressures, will be directly applicable to a DPSSL. These facilities share similar target designs, so most research on capsule and hohlraum physics for the NIF will be relevant to the development of DPSSL targets.

\subsubsection{Impact on NIF Operations, Diagnostics, or Conceptual Design}

The experiments and tests considered in this chapter have no impact on NIF operations, environment, or support facilities beyond that arising from normal NIF operations. These experiments also do not suggest modifications of the NIF conceptual design to permit these experiments to be conducted, because the normal operation of the NIF facility is the basis of these experiments. Consequently, no additional 
equipment or diagnostics are required, although it may be prudent to add certain laser performance diagnostics.

\subsubsection{Conclusions. :}

There is a direct path leading to the development of a DPSSL driver for IFE that proceeds through the NIF as an intermediate step. Although the two lasers use different gain media and different pump sources, most of their design features are similar. Validation of NIF technologies relating to such areas as optics development, optics protection, harmonic conversion, and beam transport will therefore bear directly on the design of a DPSSL. More specifically, the functional testing and operation of the NIF laser will directly validate some of the basic hardware envisioned for the DPSSL, such as the four-pass regenerative amplifier and its components. The operation of the NIF laser as a solid-state laser will therefore serve to validate the functionality of many of the components for a DPSSL driver for IFE. In this sense, the NIF-is a direct link with IFE. 


\section{6:0 IFE and Nuclear Proliferation}

\subsection{Introduction}

Significant programs on the research of ICF as an energy source are currently being conducted in both nuclear-weapon countries (e.g., US., China, Russia, and France) and in countries without nuclear weapons (e.g., Germany and Japan). The overlap in density-temperature space between nuclear weapons physics and ICF physics, and the fact that both deal with implosion systems, raise concerns that the development of IFE might increase the potential for nuclear weapons proliferation. This subject requires further analysis than we report here. However, based on our preliminary assessments, we believe that the development and commercialization of IFE will not significantly add to the risk of nuclear proliferation. Specific countermeasures are possible that limit the impact of those aspects of IFE technology that may raise concerns. The ultimate commercialization of fusion may itself be a strong counter-proliferation step.

In a manner analogous to a classification made of MFE, ${ }^{1}$ the proliferation issues for IFE can be divided into three main areas:

1. Production of fissile material for fission weapons.

2. Diversion of thermonuclear-weaponsrelevant materials tritium, deuterium, and enriched lithium.

3. Spread of thermonuclear-weapons-relevant information (computer codes, education and training, and enrichment technology). Issues regarding the first two areas are essentially the same for magnetic fusion energy and IFE. The third issue, unique to ICF, deals with using knowledge of inertial fusion implosion physics to design a thermonuclear weapon. The issues and risks, and potential solutions and/or safeguards for each area are discussed.

Of these three areas, the first area, and to a lesser degree, the second area as it relates to tritium, are the only significant concerns for proliferation. Solutions/safeguards exist for both. All other areas are insignificant due to the fact that the material is either far more efficiently drawn from another source, or the information is already available in the public domain due to other activities. They are included in this discussion only in the interest of completely addressing this topic.

\subsection{Production of Fissile Materials}

\section{Issue/Risk}

A fusion reactor could be used to produce fissile material by exposing fertile material $\left({ }^{232} \mathrm{Th}\right.$ or ${ }^{238} \mathrm{U}$ ) to the fusion neutron flux in a blanket region just outside of the fusion reaction chamber. A system designed to do this is called a fusion-fission hybrid. Many hybrid studies have been completed in the US and abroad for both MFE and IFE. 2-3 The large energy multiplication factor provided by adding fissile material to a fusion blanket improves the energy balance compared to a pure fusion design. However, the fusion-fission hybrid carries most of the same environmental and safety concerns as fission reactors. Since pure fusion offers such major potential environmental advantages over fission, and since there is not a shortage of fissile material for fission reactors (the only other reason to pursue fusion hybrids), the policy of the U.S. ICF program is to consider only pure fusion energy research with no research on fusionfission hybrids. 


\section{Solution/Safeguard}

The U.S. should take advantage of their world leadership in IFE technology and try to steer IFE research in nuclear-capable and nuclearborderline countries away from fusion hybrid research and development. Clandestine production of fissile material in fusion reactors could be curtailed by a strict monitoring and detection plan using IAEA safeguards. The $x$-ray signature of fusion-produced fissile material is easily distinguished from pure fusion power plant activation products. The alpha particle emission from actinide decay is also absent in the pure fusion case, which is perhaps the best means to assuredly discriminate as to whether a pure fusion system is being used for fissile production. All material that leaves any significant neutron flux could be sampled for alpha- or unique $\gamma$-ray emission much like screening for weapons in a metal detector. As Holdren et al., comments, ${ }^{1}$

"... it would be extremely difficult, if not impossible, for a govemment or industrial concern to achieve significant fissile material production clandestinely in an ostensibly purefusion [power plant] subject to frequent international monitoring of the sort that can be readily envisioned based on fission practice. The modification to the fusion [power plant] and its operating procedures needed to make and extract fissile material would be easy to detect, especially given that one is looking for fertile or fissile material in an environment where none is expected."

Fission reactors will always be the far easier way to produce fissile material for weapons use. Ultimately, the commercialization of fusion power will eventually remove the need for fission power. As fission technology is phased out in the latter part of the next century, a major reduction in the risks of proliferation will result.

\subsection{Diversion of Thermonuclear- Weapons-Relevant Materials}

\section{Issue/Risk}

Materials useful to nations that possess a fission weapon capability and desire a boosted or thermonuclear capability are available in fusion power plants. The materials are tritium $(\mathrm{T})$, deuterium (D) and possibly ${ }^{6} \mathrm{Li}$. Tritium diversion is the primary concern; a 1-GWe power plant will have a throughput of a couple of kilograms of tritium per day and a plant inventory of up to several kilograms. Depending on the breeding blanket design, it may be possible to produce more tritium than is consumed in the operation of the plant. This excess tritium could be diverted.

The deuterium flow rate and power plant inventory are comparable to tritium. The inventory of lithium depends on the breeding material and blanket design and could range from less than $100,000 \mathrm{~kg}$ to over $800,000 \mathrm{~kg}$. Few IFE power plant designs, however, require $\mathrm{Li}$ enriched in ${ }^{6} \mathrm{Li}$, and ${ }^{6} \mathrm{Li}$ is less important for weapons than is tritium. Deuterium and ${ }^{6} \mathrm{Li}$, while relevant to nuclear weapons, are so easily obtained through other sources that their presence in IFE will be irrelevant to the issue of proliferation.

\section{Solution/Safeguard}

Commercial fusion power plants can be subjected to materials accounting and other safeguards to prevent the possibility of diverting tritium for weapons use. One advantage that fusion has compared to fission, is that the fusion fuel cycle for recovering and recycling tritium will be entirely on the power plant site, increasing the ability to effectively secure it. The orly off-site transport of tritium that will be required will be the delivery of a startup inventory at the onset of plant operations. With regard to tritium, Holdren et al. states ${ }^{1}$ that

"... strong arguments exist for regarding the tritium safeguards issues as fundamentally less problematical for fusion than the fissile-material 
issue is for fission. Most importantly, tritium acquisition is not the limiting ingredient on thermonuclear weapon construction in the way that fissile materials are the limiting ingredient on fission weapon construction. Both the needed fission-bomb trigger and the technical insights required for design and fabrication of thermonuclear weapons are more important barriers than access to fusion fuel, and while access to tritium is convenient in thermonuclearweapon construction, it is not necessary."

As with fissile material production, the most economical and easiest-to-conceal method for a potentially proliferant country to produce tritium is with a fission reactor. It is unlikely that a nation would construct an IFE power plant solely to produce tritium.

The same type of safeguards that could be applied to tritium can be applied to deuterium and enriched lithium. Designing the fusion power plant breeding blanket with natural lithium eliminates the concern of the diversion of enriched lithium or enrichment technology, and should be pursued by IFE designers.

\subsection{Spread of}

\section{Thermonuclear-Weapons-} Relevant Information

\section{Issue/Risk}

Another concern is that the knowledge base built up from years of experience on ICF facilities, especially the NIF, and computer codes used to design ICF targets could possibly be used to aid in the design and development of thermonuclear weapons. Today, part of the main ICF design code used by U.S. researchers remains classified. However, many universities in the U.S. and several other countries have developed computer codes that can calculate ICF physics such as capsule implosions, as indicated by the partial list of non-DOE-controlled codes given in Table 6-1. A concern is that a non-nuclearweapon state could use these codes for some

Table 6-1. List of unclassified computer codes currently being use for ICF target physics.

\begin{tabular}{|c|c|c|c|c|}
\hline Code & Type & Origin & Dimensions & Comments \\
\hline ORCHID & Hydro & $\begin{array}{l}\text { University of } \\
\text { Rochester }\end{array}$ & $2-D$ & Lagrangian \\
\hline SAGE & Hydro & $\begin{array}{l}\text { University of } \\
\text { Rochester }\end{array}$ & $2-\mathrm{D}$ & Eulerian \\
\hline LILAC & Hydro & $\begin{array}{l}\text { University of } \\
\text { Rochester }\end{array}$ & $1-D$ & \\
\hline \multirow[t]{2}{*}{ NORCLA } & Hydro, & DENIM & $1-D$ & \\
\hline & $\begin{array}{l}\text { Particle Transport, } \\
\text { Implosion }\end{array}$ & & & \\
\hline SARA & Hydro & DENIM & $1-D$ & $\begin{array}{l}\text { Ion-electron, } \\
\text { Detailed radiation } \\
\text { treatment }\end{array}$ \\
\hline SITARA & Rad-Hydro & $\begin{array}{l}\text { University of } \\
\text { Frankfurt }\end{array}$ & 2-D & \\
\hline ILESTA & Rad-Hydro & Osaka & 2-D & \\
\hline HYADES & $\begin{array}{l}\text { Rad-Hydro, } \\
\text { Implosion }\end{array}$ & & $1-D$ & \\
\hline
\end{tabular}


aspect of weapon design, thereby reducing the time required to develop and acquire fission or thermonuclear weapons.

\section{Solution/Safeguard:-}

ICF codes such as listed in Table 6-1 are already widely available in the open literature. However, such codes by themselves are insufficient to enable a complete design of all aspects of thermonuclear weapons. The experimental confirmation of ICF codes that are used to model implosions of heavy-metal materials (such as $\mathrm{U}$ and $\mathrm{Pu}$ ) require experimental tests which are classified. Such experiments differ significantly from IFE target experiments (which contain no Pu or U). Since NIF and ETF IFE experimental programs will not pursue the use of any actinides (to preserve the environmental benefits of IFE), little or no experimental data from IFE target development would be available to support or confirm ICF target physics code development that could be diverted to weapon development work. Therefore, while IFE codes can have some small benefit to a proliferant country, the aid can be minimized by limiting IFE research to nonactinide targets and by continuing to deny comparisons to validated, classified ICF codes. Today, no effort to validate the IFE codes in Table 6-1 with the main ICF code is permitted. Finally, measures such as preventing access to fissile material is a far more important constraint to proliferation than access to IFE codes, since IFE codes are already widespread.

\subsection{Preliminary Conclusions}

The development and commercialization of inertial fusion energy does not significantly enhance the risks of nuclear proliferation. IFE technical information is insufficient to aid, in any substantial way, non-nuclear states to develop first generation fission weapons. Potentially sensitive areas of IFE technology (such as T diversion) can be made subject to safeguards that prevent/limit any benefit to their use by nuclear-capable states seeking to develop thermonuclear weapons.

As potential risks are identified in subsequent analyses, the specific measures that could be undertaken to neutralize or mitigate the threat could/should also be identified. Some of these measures can include research and development concerning mechanisms for bringing IFE tritium and IFE power plants under international safeguards and ensuring physical security against diversion. ICF codes that benchmark weapons specific test data will remain classified. The development of criteria for excluding collaboration with scientist and engineers from countries with poor nonproliferation credentials in facilities such as the NIF may be important as well.

\section{Reference for Section 6}

1. J. P. Holdren et al., "Report of the Senior Committee on Environmental, Safety, and Economic Aspect of Magnetic Fusion Energy," Lawrence Livermore National Laboratory, Livermore, CA, UCRL-53766 (Sept. 1989).

2 D. H. Berwald and J. A. Maniscalco, "Performance and Economics Analysis of Several Laser Fusion Breeder Fueled Electricity Generating Systems," Nucl. Technol./Fusion, 1, 137 (1981).

3. J. Maniscalco, "Fusion-Fission Hybrid Concepts for Laser-Induced Fusion," Nucl. Technol., 28, 98 (1976). 


\subsection{Conclusions}

The NIF has the capability to address many of the critical IFE issues that are attendant to the design of ETF and future IFE power plants. Most important is the demonstration of inertial fusion ignition and gain in the NIF, which is a key prerequisite for IFE and for defense. In addition, the NIF, designed to support ICF ignition and gain for its primary defense mission, provides flexibility to support a broad range of IFE-specific experiments in the areas of IFE target optimization physics, chamber clearing and gas dynamics, data for IFE materials damage response, operational experience in many target area systems prototypical of IFE power plants, demonstration of safe and reliable management of radioactive materials, and a test environment for prototype IFE targets and injection systems. These possibilities have generated great interest in the fusion energy community as ideas to implement them have emerged, and DOE Energy Research planning to support development for future IFE experiments on the NIF has begun. DOE recognizes the NIF to be a national user facility capable of conducting unclassified IFE experiments along with other uses of NIF. The exciting opportunities for IFE experiments on the NIF have boosted the hopes of many that IFE can indeed be developed in a timely and costeffective manner.

Many of the proposed IFE experiments would benefit from current testing on Nova as a precursor to NIF, with NIF providing the final integration tests. Some NIF-IFE experiments need initial development off-line before they would be ready for tests on the NIF. More effort is required to better define design details of these NIF-IFE experiments, such as the code-predicted responses of these experiments in the NIF chamber, the specific diagnostic information to be obtained, and needed developments of appropriate IFE chamber dynamic and materials response codes that would be used to model the resulting IFE data. An IFE national users group will need to be formed to formulate and prioritize the IFE developments leading to the NIF. These activities will be needed prior to NIF ignition, to be ready to expand IFE experiments on the NIF once ignition is demonstrated, and to provide the technical basis for a timely initiation of an ETF facility.

\section{Acknowledgments}

The authors would like to acknowledge the contributions of the 60 participants who attended the workshop at UC Berkeley February 22-24, 1994, who provided the many ideas forming the basis of this paper. In particular, we thank Per Peterson and Ralph Moir for their help with chamber dynamics and fusion power technology respectively. This work was performed under the auspices of the U.S. Department of Energy by Lawrence Livermore National Laboratory under contract W-7405-Eng-48. 